\title{
Low-Cost Skewed Redundant IMU Configuration for State-Space Recovery in a Saturated Environment
}

\author{
Levi S. Hubbard \\ West Virginia University, Ishubbard@mix.wvu.edu
}

Follow this and additional works at: https://researchrepository.wvu.edu/etd

Part of the Aerodynamics and Fluid Mechanics Commons, Aeronautical Vehicles Commons, and the Navigation, Guidance, Control and Dynamics Commons

\section{Recommended Citation}

Hubbard, Levi S., "Low-Cost Skewed Redundant IMU Configuration for State-Space Recovery in a Saturated Environment" (2020). Graduate Theses, Dissertations, and Problem Reports. 7814. https://researchrepository.wvu.edu/etd/7814

This Thesis is protected by copyright and/or related rights. It has been brought to you by the The Research Repository @ WVU with permission from the rights-holder(s). You are free to use this Thesis in any way that is permitted by the copyright and related rights legislation that applies to your use. For other uses you must obtain permission from the rights-holder(s) directly, unless additional rights are indicated by a Creative Commons license in the record and/ or on the work itself. This Thesis has been accepted for inclusion in WVU Graduate Theses, Dissertations, and Problem Reports collection by an authorized administrator of The Research Repository @ WVU. For more information, please contact researchrepository@mail.wvu.edu. 


\title{
LOW-COST SKEWED REDUNDANT IMU CONFIGURATION FOR STATE-SPACE RECOVERY IN A SATURATED ENVIRONMENT
}

\author{
Levi Hubbard
}

\author{
Thesis submitted to the \\ Benjamin M. Statler College of Engineering and Mineral Resources \\ at West Virginia University \\ in partial fulfillment of the requirements for the degree of \\ Master of Science \\ in \\ Aerospace Engineering
}

Patrick Browning, Chair, Ph.D.

Wade Huebsch, Ph.D.

Christopher Griffin, Ph.D.

Department of Mechanical and Aerospace Engineering

Morgantown, West Virginia

2020

Keywords: Low-Cost, IMU, Redundant, State-Space, Experimental

Copyright Levi S. Hubbard(C) 2020 


\title{
ABSTRACT \\ Low-Cost Skewed Redundant IMU Configuration for State-Space Recovery in a Saturated Environment
}

\author{
Levi Hubbard
}

Low-cost sensors for state space determination can be used successfully for ground vehicles, robots, unmanned aerial vehicles, and Internet-of-Things applications. When a high fidelity Inertial Measurement Unit (IMU) cannot be obtained for state space determination, low-cost sensors can be used to satisfactory standards, despite their limitations in capabilities, by using various implementation techniques. The research group was experimentally investigating state space information of an unstable flying vehicle for motion simulation validation. The high fidelity motion capture system would intermittently lose track of the flight vehicle which lost critical flight data.

The goal was to determine the potential of low-cost off-the-shelf sensors to provide a lower fidelity backup source of data. There were periods during the flight test where the flying vehicle was known to experience rotation rates higher than the saturation limit of the low-cost sensors. The purpose of the experiment was to analyze the ability of a skewed-redundant IMU (SRIMU) configuration to extend the dynamic range of the MEMS gyroscope and to reconstruct body axis rotation rates that would have otherwise been saturated.

The experiment was able to determine the potential of low-cost off the shelf IMU sensors in a skewed redundant IMU configuration to reconstruct saturated values. There was success in extending the dynamic range of the sensors in cases where a rotation matrix could be utilized to transform data between reference frames. However there were instances where the dynamic range could not be extended due to relative differences in time between sensors which incurred over the duration of the flight tests. 


\section{Acknowledgments}

A humble gratitude to Dr. Browning who facilitated the ability to continue my education by joining his research team. His hard work and dedication will be an example for all my future endeavors.

My committee members, Dr. Wade Huebsch and Dr. Christopher Griffin. For working along side them on the project.

My colleague Dr. Joe Dygert for help in the lab and mentorship in my research.

Special thanks to John Kantelis from Systems Engineering Group for working on this exciting project together.

My wife, my strength, who among all was the most instrumental in keeping me going through engineering. The sleepless nights, the long days, would not have been achievable without her.

soli deo gloria 


\section{Dedication}

To my Father,

Blessed be the lord our God! Whom I count it all joy for the blessings laid out upon my life, you Father, being chief among them.

The cycles that transcend generations are not often nor are they easily broken, yet standing before me is a father figure that broke the mold and dedicated his life to being the most tender-hearted and caring man he could be. I couldn't hope, in my wildest dreams, to follow in your footsteps. I should count it a success to be half of what you are, be that even attainable.

My early memories consist of being by your side, trowel in hand, ready to help spread mortar for your bricks. Or excitedly waiting for you to come home from work so we could all eat pecan rolls together. Irregardless of how exhausted you were, it was a party when Dad came home from work.

I will be forever grateful for the strong character you shaped and molded in me. You taught me how to have a strong work ethic as I grew up, how to be kind to others, and how to be gentle to those we love.

If I've learned anything from you, it is that there is no higher calling in life than to sacrifice for your family, in any capacity. A successful life is worn hands, a tired back, and your family around you.

We've always been best friends, and we always will be.

I love you 


\section{Contents}

$\begin{array}{ll}\text { Abstract } & \text { ii }\end{array}$

Acknowledgments $\quad$ iii

Dedication $\quad$ iv

List of Figures $\quad$ vii

List of Tables $\quad x$

1 Introduction 1

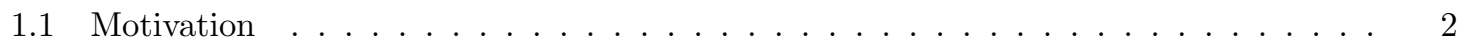

1.2 Historical Relevance . . . . . . . . . . . . . . . . . . . . . . . 3

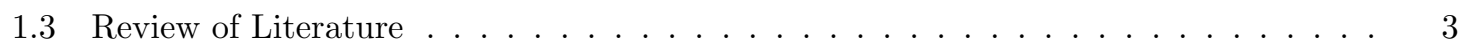

1.3.1 Redundant Systems . . . . . . . . . . . . . . . . . . . . . . . 3

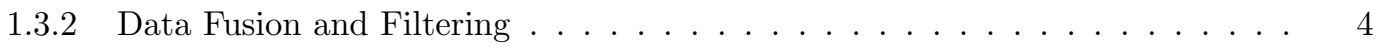

1.3.3 Low Cost Sensors . . . . . . . . . . . . . . . . . . . . . . . 6

1.3.4 Attitude Representation . . . . . . . . . . . . . . . . . . 6

1.4 Proposed Research . . . . . . . . . . . . . . . . . . . . . . . . 7

2 Methodology $\quad 8$

2.1 IMU Sensor Information . . . . . . . . . . . . . . . . . . . . . . 9

2.2 Relative Motion . . . . . . . . . . . . . . . . . . . . . . . . . . . . 10

2.3 Rotation Matrices . . . . . . . . . . . . . . . . . . . . . 12

2.4 Genetic Algorithm Skew Angles . . . . . . . . . . . . . . . . . . . . . . . . 14

2.5 Flight Test Payload . . . . . . . . . . . . . . . . . . . . . . 20

2.6 Wind Tunnel Test Payload _ . . . . . . . . . . . . . . . . . . . . . . . . 22 
2.7 Vicon Motion Capture . . . . . . . . . . . . . . . . . . . . . . . . . . . 23

2.8 Test Setup . . . . . . . . . . . . . . . . . . . . . . . . . . . . . . 27

2.9 Post-Processing Data . . . . . . . . . . . . . . . . . . . . . . . . . . . 29

$\begin{array}{llr}3 & \text { Results } & 31\end{array}$

3.1 Genetic Algorithm . . . . . . . . . . . . . . . . . . . . . . 31

3.2 Sensitivity Analysis . . . . . . . . . . . . . . . . . . . . . . . . . 31

3.3 Uncertainty Analysis . . . . . . . . . . . . . . . . . . . . . . . 33

3.4 Flight Test . . . . . . . . . . . . . . . . . . . . . . . . . . 36

3.4.1 Run $1 \ldots \ldots \ldots \ldots \ldots$

3.4 .2 Run $2 \ldots \ldots \ldots \ldots 1$

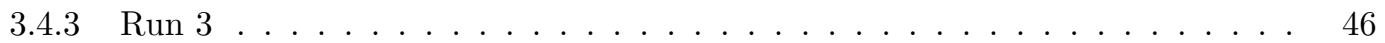

3.4 Run $4 \ldots \ldots \ldots \ldots \ldots$

3.4 .5 Run $5 \ldots \ldots \ldots \ldots \ldots 4$

3.5 Discussion . . . . . . . . . . . . . . . . . . . . . . . . . . 59

4 Conclusions \& Future Development 62

\begin{tabular}{l} 
Appendix A \\
\hline
\end{tabular}

$\begin{array}{lr}\text { Appendix B } & 66\end{array}$

$\begin{array}{lr}\text { Appendix C } & 67\end{array}$

$\begin{array}{lr}\text { Appendix D } & 68\end{array}$ 


\section{List of Figures}

2.1 Sparkfun 9DOF Razor IMU. Source https://learn.sparkfun.com/tutorials/9dof-razor-

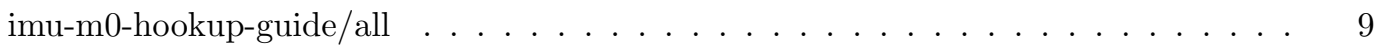

2.2 Visualization of relative angular rotation as described through skew angle $\theta \ldots \ldots 11$

2.3 Augmented Matrix reconstruction for reference frame transformation . . . . . . . . . 14

2.4 Monte Carlo simulation noting probability of recovering saturated rotation rates for

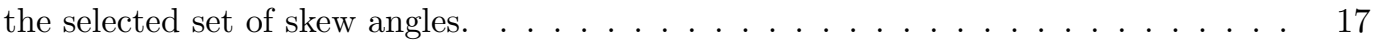

2.5 Monte Carlo simulation noting probability of recovering saturated rotation rates for cases of one saturated channel, two saturated channels, and three saturated channels on the body axis . . . . . . . . . . . . . . . . . . . . 18

2.6 Monte Carlo recovery rate process _ . . . . . . . . . . . . . . . . . . . . . . . . 19

2.7 Initial effort to place skewed IMU platforms within internal geometry constraints . . 20

2.8 Instrumentation payload showing tungsten ballasts and battery pack . . . . . . . . 21

2.9 Instrumentation payload with IMUs wired together and placed in the tail cone of the flight vehicle . . . . . . . . . . . . . . . . . . . . . . . . . 21

2.10 IMU sensor holder . . . . . . . . . . . . . . . . . . . . . . . . . . . 23

2.11 Vicon motion capture calibration wand. Photo: WVU-AJ Research Project . . . . . 24

2.12 Example design of reflective Vicon marker placement establishing Euclidean distance

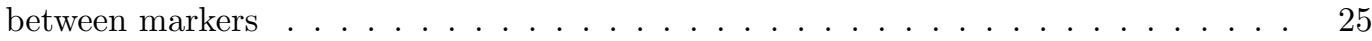

2.13 Final reflective marker configuration designed for the wind tunnel instrumentation

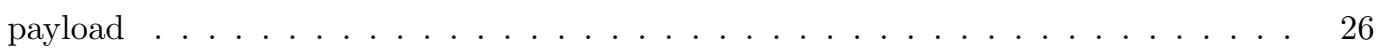

2.14 Reedsville, WV. Wind tunnel test setup . . . . . . . . . . . . . . . . . 27

2.15 Wind tunnel instrumentation payload IMU holder wired for testing . . . . . . . . . 28 
2.16 Wind tunnel illustration visualizing the arresting system on the left hand side, the motion capture cameras along the top, and a typical curved flight path of the test payload starting from right to left. . . . . . . . . . . . . . . . . . . . 29

3.1 Cross section representation of 3D printed uncertainty due to layer thickness and the maximum difference possible $\ldots \ldots \ldots \ldots \ldots \ldots \ldots$

3.2 Run 1: Frequency domain analysis for temporal alignment of sensors . . . . . . . . 37

3.3 Run 1: Relative IMU ROI data . . . . . . . . . . . . . . . . . . . . . . 38

3.4 Run 1: IMU data as recorded (a) and after conversion to common reference frame (b) 38

3.5 Run 1: Rotation about the $\mathrm{x}$ axis $\ldots \ldots \ldots \ldots \ldots$

3.6 Run 1: Rotation about the y axis . . . . . . . . . . . . . . . . 39

3.7 Run 1: Rotation about the $\mathrm{z}$ axis . . . . . . . . . . . . . . . . . . . 40

3.8 Run 1: Converted IMU data and Kalman Filter data fit . . . . . . . . . . . . . . . 40

3.9 Run 2: Frequency domain analysis for temporal alignment of sensors . . . . . . . . 41

3.10 Run 2: Relative IMU ROI data . . . . . . . . . . . . . . . . . . . . . . . 42

3.11 Run 2: IMU data as recorded (a) and after conversion to common reference frame (b) 42

3.12 Run 2 : Rotation about the $\mathrm{x}$ axis $\ldots \ldots \ldots \ldots \ldots$

3.13 Run 2: Rotation about the y axis . . . . . . . . . . . . . . 43

3.14 Run 2: Rotation about the $\mathrm{z}$ axis $\ldots \ldots \ldots \ldots \ldots \ldots$

3.15 Run 2: Converted IMU data and Kalman Filter data fit . . . . . . . . . . . . . 44

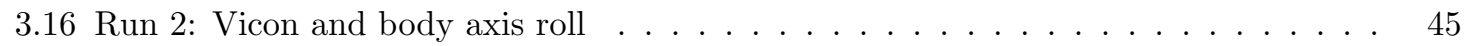

3.17 Run 3: Frequency domain analysis for temporal alignment of sensors . . . . . . . . 46

3.18 Run 3: Relative IMU ROI data . . . . . . . . . . . . . . . . . . . . . . . . . 47

3.19 Run 3: IMU data as recorded (a) and after conversion to common reference frame (b) 47

3.20 Run 3 : Rotation about the $\mathrm{x}$ axis $\ldots \ldots \ldots \ldots$. . . . . . . . . . 48

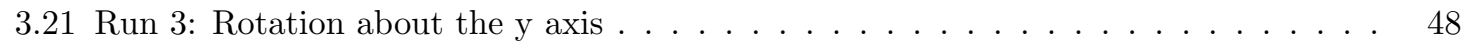

3.22 Run 3: Rotation about the $\mathrm{z}$ axis $\ldots \ldots \ldots \ldots$

3.23 Run 3: Converted IMU data and Kalman Filter data fit . . . . . . . . . . . . . . . 49

3.24 Run 4: Frequency domain analysis for temporal alignment of sensors . . . . . . . . 50

3.25 Run 3: ROI data . . . . . . . . . . . . . . . . . . . . . 51

3.26 Run 4: IMU data as recorded (a) and after conversion to common reference frame (b) 51

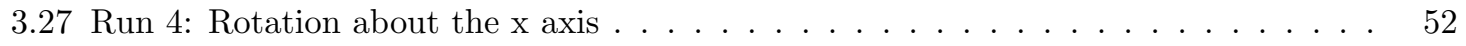

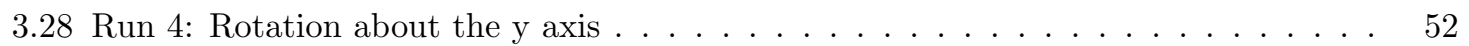


3.29 Run 4 : Rotation about the $\mathrm{z}$ axis . . . . . . . . . . . . . . . . . 53

3.30 Run 4: Converted IMU data and Kalman Filter data fit . . . . . . . . . . . . . 53

3.31 Run 5: Frequency domain analysis for temporal alignment of sensors . . . . . . . . 54

3.32 Run 5: Relative IMU ROI data . . . . . . . . . . . . . . . . . . . . . . 55

3.33 Run 5: IMU data as recorded (a) and after conversion to common reference frame (b) 55

3.34 Run 5 : Rotation about the $\mathrm{x}$ axis $\ldots \ldots \ldots \ldots \ldots$

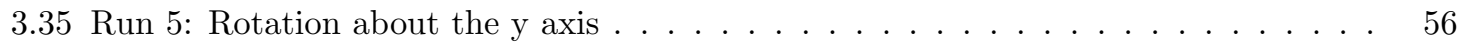

3.36 Run 5 : Rotation about the $\mathrm{z}$ axis $\ldots \ldots \ldots \ldots \ldots \ldots$. . . . . . . . . . 57

3.37 Run 5: Converted IMU data and Kalman Filter data fit . . . . . . . . . . . . . . 57

3.38 Run 5 : Rotation about the $\mathrm{x}$ axis $\ldots \ldots \ldots \ldots \ldots \ldots$

4.1 Hand drawn calculations for marker placement . . . . . . . . . . . . . . . . 65

4.2 Euclidean distance calculation considering all permutations between reflective markers. Measurements were in inches. . . . . . . . . . . . . . . . . 66 


\section{List of Tables}

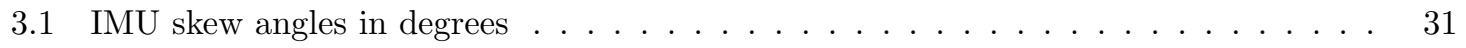

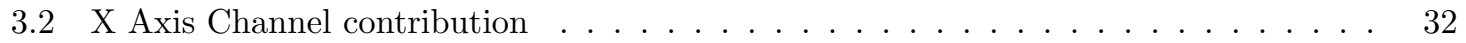

3.3 Y Axis Channel contribution . . . . . . . . . . . . . . . . . . . . . . 32

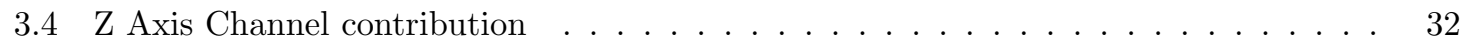

$3.5 \quad \mathrm{X} \& \mathrm{Y}$ Axis Channel contribution $\ldots \ldots \ldots \ldots \ldots \ldots$

3.6 Y \& Z Axis Channel contribution . . . . . . . . . . . . . . . . . . . . 33

$3.7 \quad \mathrm{X} \& \mathrm{Z}$ Axis Channel contribution . . . . . . . . . . . . . . . . . . . . . . 33

$3.8 \quad \mathrm{X}, \mathrm{Y}, \& \mathrm{Z}$ Axis Channel contribution . . . . . . . . . . . . . . . . . . 33

3.9 sine and cosine relative uncertainty $\ldots \ldots \ldots \ldots \ldots \ldots$ 


\title{
Acronyms
}

$\begin{array}{ll}\text { Symbol } & \text { Description } \\ \text { BA } & \text { Body Axis } \\ \text { DOF } & \text { Degrees of Freedom } \\ \text { EKF } & \text { Extended Kalman Filter } \\ \text { FAA } & \text { Federal Aviation Association } \\ \text { FFT } & \text { Fast Fourier Transform } \\ \text { GA } & \text { Genetic Algorithm } \\ \text { GPS } & \text { Global Positioning System } \\ \text { INS } & \text { Inertial Navigation System } \\ \text { IMU } & \text { Inertial Measurement Unit } \\ \text { SLA } & \text { Stereo lithography } \\ \text { SRIMU } & \text { Skewed Redundant Inertial Measurement Unit } \\ \text { UAV } & \text { Unmanned Aerial Vehicle } \\ \text { UAS } & \text { Unmanned Aerial System } \\ \text { UKF } & \text { Unscented Kalman Filter } \\ \text { dps } & \text { Degrees Per Second }\end{array}$

\section{Nomenclature}

\author{
Symbol Description \\ PQR Yaw, Pitch, and Roll \\ local notes the measurement relative to an IMU \\ ba notes the measurement relative to the IMU aligned with the body axis
}




\section{Chapter 1}

\section{Introduction}

State space determination is the measurement of the current physical configuration of a system. State information is important because it describes the behavior of the system being measured at a given time. the measurements of a system can effectively be captured through the implementation of low-cost sensors. Although there can be limitations to their capabilities, low-cost sensors can be used to adequate standards for applications such as ground vehicles, robotics, IoT (Internet of Things), and unmanned aerial vehicles. Because of the price, typical ease of implementation, and abundant supporting documentation, they can be beneficial to individuals, student design teams, start-ups, or research teams that are measuring the behavior of an object.

In the experimental work performed, state information of a flying vehicle was measured in order to validate motion simulations. The flight data was used to reconstruct the aerodynamic coefficients to better tune the model of the flight vehicle motion simulation. For the experiment, motion capture was used as the primary data acquisition system to record body rotation rates of the flight vehicle. Due to the unstable characteristics of the flight vehicle, there were rapid transitions between attitude orientations which in turn caused the motion capture system to intermittently lose track of the flight vehicle and lose critical flight data.

The goal was to create a secondary source of lower fidelity data for the experiment that could be used to fill in the missing regions of data. Due to the limitations of the sensor capabilities, a method was developed to extend the dynamic range of the MEMS gyroscope. The low-cost sensors were placed in a skewed redundant configuration to leverage the relative difference in coordinate frames to extend the dynamic range of the sensors. This method allowed the capabilities of readily accessible sensors to be extended so that they could be used in place of otherwise expensive hardware. 


\subsection{Motivation}

The researchers experienced a loss of state-space information of a flying vehicle during flight tests. Complexities of the unstable aerodynamic behavior would intermittently cause the primary motion capture data acquisition system to briefly lose track of the flying vehicle, losing critical flight information.

The research group investigated the characteristics of an unstable flying vehicle to provide validation to simulations. The aerodynamic coefficients of the flight vehicle were extracted from the flight data to provide an improved simulation of the model. The experiment was carried out by launching a scaled model of the flying vehicle from a pneumatically actuated cannon. The flight was observed from the initiation of the launch until the scale model was stopped in an arresting net approximately one hundred yards down range. The primary data acquisition system used to record data of the experiment was a motion capture system that allowed the observation of the flying vehicle without introducing external factors into the experiment.

Preliminary results showed the flight vehicle would rapidly transition between attitude orientations in a manner that caused the motion capture system to intermittently lose track of the flight vehicle. The loss of data created regions of the test with no state-space information. The lack of data of the flight vehicle required additional efforts to provide a source of backup data that could be used to fill in the regions of the flight data with no state-space information.

It was determined that Inertial Measurement Units (IMUs) would be used to provide a backup source of data to the primary data acquisition system. An IMU sensor system that would be quickly and efficiently integrated into the flight vehicle was desired. Therefore, an off-the-shelf sensor was chosen for ease of implementation. Additionally, it was desired that the secondary data acquisition system was readily affordable.

Because the desires for the secondary data acquisition system were affordability and ease of implementation, the sensors available had limitations in their capabilities. There was increased risk

of failure due to the quality of sensors. And it was known that the rotation rates experienced by the flight vehicle was higher than the nominal range of the sensor. Therefore, the secondary data acquisition system needed a source of redundancy and a method to increase the dynamic range of the MEMS-gyroscope. 


\section{$1.2 \quad$ Historical Relevance}

Inertial Measurement Units (IMUs) are systems constructed of one or more sensors that sense change in angular displacement, angular velocity, or acceleration. Gyroscopes specifically, a type of sensor in an IMU, measure angular velocity through a natural phenomenon known as precession. First discovered by early Greeks, Chinese, and Romans, this phenomenon was observed through spinning tops as toys. The occurrence was understood, albeit with little to no practical use. Serson, an Englishman in the 1740's observed spinning tops' ability to remain upright despite the movement of the surface it rotated upon [1]. He proposed it could be used as an artificial horizon for ships but was not able to test his idea. The term 'gyroscope' was cemented by French physicist Leon Foucault in 1852. He first studied the rotation of the earth through motion of a pendulum, and observed the change in direction as the earth rotated below it. He later studied a high-speed spinning top placed inside a ring and wheel. During a twenty-four-hour period, the axis came back to point at the original starting position. In the early twentieth century, German inventor Hermann AnschutzKaempfe created the gyrocompass in his polar expedition endeavors. The practical implementation of the gyroscope, the gyrocompass, had the ability to measure relative angular motion and display it in a meaningful way. This allowed the implementer a measure of feedback from a reference orientation in three-dimensional space [2]. The further practical implementation of gyroscopes saw important usage as the world prepared for WWII. The advancement in technology due to the war, propelled the integration of gyroscopes into axis and allied aircraft to maintain heading as the endurance of aircraft increased. Mechanical gyroscopes also found themselves in the bombardier's optics for timing the release of ordnance. With the use of gyroscopes for navigation firmly cemented from the theatre of war, Inertial Navigation Systems (INS) have continued evolving to the present. In addition to gyroscopes, accelerometers and GPS have been combined to create a robust system of navigation.

\subsection{Review of Literature}

\subsubsection{Redundant Systems}

An electromechanical device will inevitably fail partially or completely at some point during its life cycle. In the effort to preserve integrity and increase performance, it is necessary to incorporate more than one type of inertial measurement sensor. Multiple sensors provides fault tolerance against failures and also provides higher confidence in measurement. When utilizing lower quality low-cost 
sensors, multiple sensors provide a backup source of data due to the redundancy.

There are several methods in which to configure a redundant navigation system. Generally speaking, IMUs have three primary axes set orthogonal to each which represent the three dimensions. Clustering several sensors together in an orthogonal or parallel manner with their primary axes aligned is a basic configuration that lends itself readily to fault detection as the integration of the signals together is straight forward. Clustering multiple sensors together with their principle axes in a non-orthogonal or Skewed Redundant Inertial Measurement Unit (SRIMU) configuration can increase the accuracy of the overall system [3].

Sukkarieh's [4] continued work makes the case for redundant low cost inertial navigation systems such that the civilian population can readily benefit from their implementation. His work showed low cost sensors can be configured to provide fault detection and are suitable for unmanned aerial vehicles and pertinent safety requirements. Guerrier [5] created a tool to help researchers and system designers understand the number of sensors and their relationship to performance improvement. He also created metrics demonstrating the number of sensors necessary for certain mission specifications and the impact of sensor orientation on performance. Early work by Pejsa in 1974 [6] explored the geometry involved in orienting sensors. His research postulated placing $n$ number of sensors on a cone with a half angle $\alpha$ where the uncertainty of the configuration is minimized. He further determined the total number of sensors required for fault detection and isolation (FDI). A minimum number of four sensors are required for fault detection and a minimum of five sensors are required for fault isolation. Finally, Colomina [7] et. al used a redundant IMU configuration to enhance precision in airborne applications. They explored using a SRIMU system to reduce uncertainty in the quality of navigation parameters like heading and trajectory. Research shows redundant IMU configurations have been leveraged for benefits in a broad swath of industries. Redundant systems help improve accuracy [8], provide means for fault tolerance and fault detection [9].

\subsubsection{Data Fusion and Filtering}

Data that is measured by sensors are inherently clouded by noise, that is to say, they display a band of measurements around the actual signal. Methods have been developed to reduce the uncertainty around the measurement such that there is a higher confidence in the measured signal. Additionally, methods are needed to combine data from multiple types of sensors.

Data fusion as defined by Bostrom, et. al [10] is the "Information fusion is the study of efficient methods for automatically or semi-automatically transforming information from different sources and 
different points in time into a representation that provides effective support for human or automated decision making". Fusing of information is important to create a complete understanding. There can be more than one type of the same sensor such that there is a redundant source of a certain type of information. Additionally multi-sensor configurations can be used with different sensors such as GPS (global positioning system), gyroscope, accelerometer, etc. It is necessary to have a method that takes the different values from the different types of sensors and combines them in a manner that makes sense.

Generally, there are three categories which data fusion could be grouped in. The first is complementary which combines independent sensors which do not directly depend on each other but create a whole picture. Second is competitive where independent sensors deliver measurements of the same behavior, also called a redundant system. Lastly, cooperative where two independent sensors have their measurements used to create new information that a single sensor could not do on its own [11].

Multi sensor configurations are becoming more prevalent in military and non-military applications. Hall and LLinas [12] made the case analogous to humans acquiring a more accurate assessment of their surroundings based on multi sensory inputs so to other applications infer a more accurate understanding of the surroundings and subsequently make better decisions based on the higher level of information fused together. Additional work [13] showcases an important application for a UAV tasked with monitoring wild fires. The GPS, inclinometer, and video camera must be fused together to provide information regarding 3D mapping.

Whether working with single sensors, multiple sensors or multiple sensor types, there will be an uncertainty based around the measurement. This is true for any measurement taken by a sensor. Different techniques exist to help reduce the uncertainty around the signal. The techniques generally depend on the nature of the data. For example, Kalman filtering is a method to help reduce random process noise. There are however different types of Kalman filtering based on the behavior of the signal. A system that behaves linearly can be addressed by the baseline Kalman filtering process. Nonlinear system behavior can be filtered by the extended Kalman filter (EKF). Nonlinear function filtration can be evaluated by the unscented Kalman filter (UKF).

Filtration and fusion are needed when working with a redundant system with multiple sensors. If the sensors are of the same type, then the most simple case can be to filter and combine them together for comparison. Different types of sensors can have their data combined to accomplish a common goal [14]. 


\subsubsection{Low Cost Sensors}

Inertial Navigation Systems (INS) are inherently prone to uncertainties because their signals are either integrated or differentiated. Subsequently, they experience 'drift'. Drift refers to a signal that indicates a change in motion when in reality the system being measured is physically at rest. The range and sensitivity of a quality sensor are inversely proportional, as the range of the sensor increases, the sensitivity of the sensor decreases. That is because there is a given bit depth which describes resolution capable. If there is a wider range of values, that resolution is spread over a wider range of numbers and the sensitivity is decreased. In commercial aviation which has high fidelity standards handed out by the FAA [15], high precision IMUs are implemented which have low drift and low noise. However they are significantly expensive. Conversely, in the hobby market, with sensors often in the hundred dollar range, sensors are prone to high drift rates and noise.

Low cost sensors are desirable for products in mass markets states Gonzalez [16]. They can be used for IoT (Internet of Things) and wearables for example. Low-cost sensors are also gaining popularity in use in ground vehicles where an ultra low-cost IMU sensor was used satisfactorily with a GNSS receiver for vehicle navigation. Due to the increasing performance capabilities of the low-cost IMU sensors, these IMUs require consideration when designing system navigation instrumentation.

Low cost sensors are important for the use of unmanned aerial vehicles where weight and price are important design criteria. With necessary implementation techniques and sensor algorithms, the low-cost sensors can be used adequately to provide attitude estimation [17]. Low-cost sensors have also been shown to be useful in guidance, navigation, and control for unmanned aerial vehicles. The unmanned vehicle would be able to provide real time flight data that would be fed back into the control loop to provide important information for the autonomous control system to operate. The capability of low-cost sensors to provide adequate guidance, navigation, and control will provide an avenue for an increase in civilian operation of autonomous unmanned aerial vehicles [18].

\subsubsection{Attitude Representation}

When recording attitude data in a Cartesian coordinate frame with Euler angles, gyroscopes are susceptible to the phenomenon called gimble lock. Gimble lock happens when two rings of the gyroscope line up in phase with each other and the number of dimensions recordable reduce from three dimensions down to two dimensions. Using PQR notation (yaw, pitch, and roll), gimble lock occurs when the pitch angle reaches ninety degrees. In the research performed, the UAV during flight often completed full rotations over all three axes and would have experienced multiple instances of 
gimble lock.

An alternative method to record attitude data is to use quaternions. Hamilton, in 1843, developed the concept of quadruplets such to describe three dimensional geometry in an analogous manner as to that of couplets in complex numbers [19] [20]. Quaternions do not have areas that present singularities and can therefore record any orientation of attitude without loss of data. Quaternions are a way in which to describe space in four dimensions instead of three. When describing a rotation in four dimensions, the axis the body rotates about is described as the three dimensional $x, y$, and $z$ vector that pierces the center of mass of an object that it is rotated about. The fourth term describes the amount in radians by which the body rotates around the $x y z$ vector. The vector portion is referred to as the imaginary part and the real part is the rotation angle $w$.

\subsection{Proposed Research}

The research carried out determined the potential of low-cost sensors to provide critical backup data to a primary motion capture data acquisition system and develop an implementation technique to increase the dynamic range of the low-cost MEMS gyroscope. To create a backup source of data, the IMUs were clustered in a skewed configuration to provide a source of fault tolerance by having redundant sensors. The geometrical relationship of the Skewed Redundant IMU (SRIMU) configuration was leveraged to develop a method that extended the dynamic range of the sensors. 


\section{Chapter 2}

\section{Methodology}

The rationale for the low-cost sensors chosen will be discussed and an overview of the time synchronization between sensors will be given, followed by an explanation of the method developed to extend the dynamic range of the sensors with lessons learned. The mathematical relationship between the skewed reference frame and how that was leveraged to support the developed method will be demonstrated. The design and implementation of a genetic algorithm for skew angle selection will be discussed and the estimated recovery of saturated values will be postulated. The design of the instrumentation of the payload for the original research effort will be shown as well as the instrumentation payload for the wind tunnel test. Finally the wind tunnel setup and test environment will be explained. 


\subsection{IMU Sensor Information}

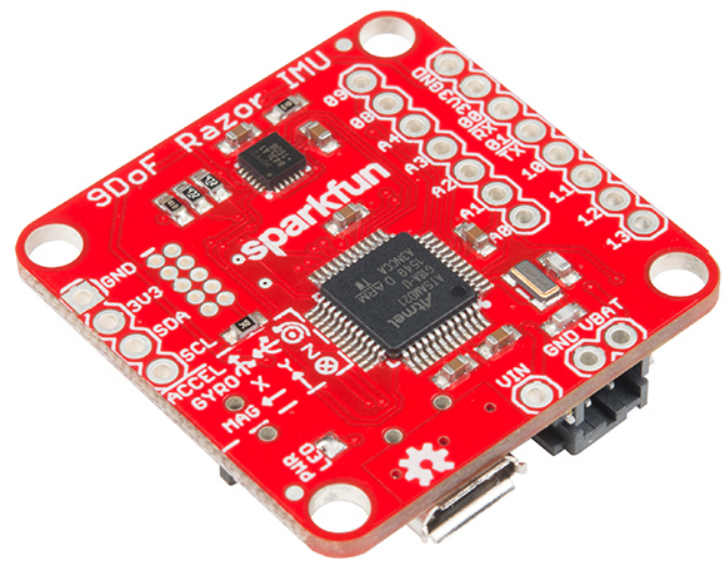

Figure 2.1: Sparkfun 9DOF Razor IMU. Source https://learn.sparkfun.com/tutorials/9dof-razorimu-m0-hookup-guide/all

The sensor selected for use was the Razor 9DOF IMU. It had an MPU-9250 IMU with acceleration range of $\pm 16 \mathrm{~g}$ and rotation rate range of \pm 2000 degrees per second. The system could record data at a sampling rate of $100 \mathrm{~Hz}$. The sensor board used an Atmel SAMD21 microprocessor.

There were several reasons the specific sensors chosen were low-cost off the shelf components from Sparkfun. One of the reasons was based on familiarity with that platform. There was previous work done documenting the capabilities of that specific sensor. Because it was known how to use the features of the board there was not a need to learn a new software interface or develop a new understand of the hardware.

A second reason for the specific sensor was based on the support for the IMU. The supplier Sparkfun had a substantial amount of documentation illustrating how to implement and utilize the features of the sensor board. There was also a substantial presence on forums that discussed trouble shooting potential bugs that could be encountered.

Because there were four individual sensor boards, configuration of time synchronization was a necessary feature of the system. The sensor boards could use $i^{2} c$ (inter-integrated circuit) and SPI (Serial Peripheral Interface) communication protocols according to the sensor documentation. SPI was the first method implemented, however it was discovered that there was not enough GPIO (general purpose input/output) pins that was required for the hardware implementation. Connecting 
four boards using SPI wouldn't work, which lead to implementing $\mathrm{i}^{2} \mathrm{c} . \mathrm{i}^{2} \mathrm{c}$ required less wires as compared to SPI during hardware implementation, however it was discovered that the MPU-9250 IMU chip and the SAMD-21 microprocessor used the $\mathrm{i}^{2} \mathrm{c}$ bus to send data back and forth which rendered that method unusable. Finally a simple solution to use the analog pins on each board to communicate was implemented. The IMU aligned with the body axis would send an analog signal to the skewed IMUs to begin logging data. However the analog signal method did not completely align the data and further time synchronization was needed in post processing.

\subsection{Relative Motion}

The limitations of the low-cost sensors meant the dynamic range of the MEMS gyroscope was less than required. The flight vehicle was expected to experience rotation rates higher than the two thousand degree per second sensor range. Due to the short comings, a method to extend the dynamic range of the sensors was necessary. The original purpose of SRIMU systems provided redundancy and increased precision through the relative difference in coordinate axes. The method developed here involved leveraging that relative difference in coordinate axes to increase the dynamic range of the MEMS gyroscopes.

The coordinate axes were labeled such that The body axes of the UAV was considered to be the common reference frame. The body axis was described in three dimensions by the roll, pitch, and yaw, corresponding to the $x, y$, and $z$ respectively. Three additional IMU reference frames were configured, each having their own orthogonal three dimensional axes. The IMUs configured together were then skewed some angle in degrees with respect to the body axes.

A method was developed then to monitor the relative motion measured and tune it such that the skewed IMUs could experience body axis rotation rates in an unsaturated condition. The goal being, the skewed IMUs having the ability to record data during an instance when the IMU aligned with the body axis would be in a saturated state. Therefore, the axes of the skewed IMUs would see reduced angular roll rates as compared to what the body axis would actually be experiencing.

The MEMS gyroscopes were able to capture body axis rotation rates higher than the sensors manufactured saturation limits through relative motion. The axes of a three dimensional reference frame, or even more simply, a two dimensional reference frame, would observe some portion of motion relative to that of another two dimensional reference plane that is not parallel to the first. The concept can be visualized in Figure 2.2. With a pure rotation rate about the $x_{1}$ axis, the rotation rate experienced by $x_{2}$ would be the cosine of the skew angle $\theta$. Extending the notation to 
a three dimensional example, the rotation rates that acted on a common reference frame would be experienced by the second reference frame as a function of the trigonometric relationship between the two reference frames. The goal for the SRIMU system was for the skewed IMU sensors to be able to experience data below the saturation limit while the body axes would otherwise be in a state of saturation; experiencing rotation rates above the two thousand degree per second limit.

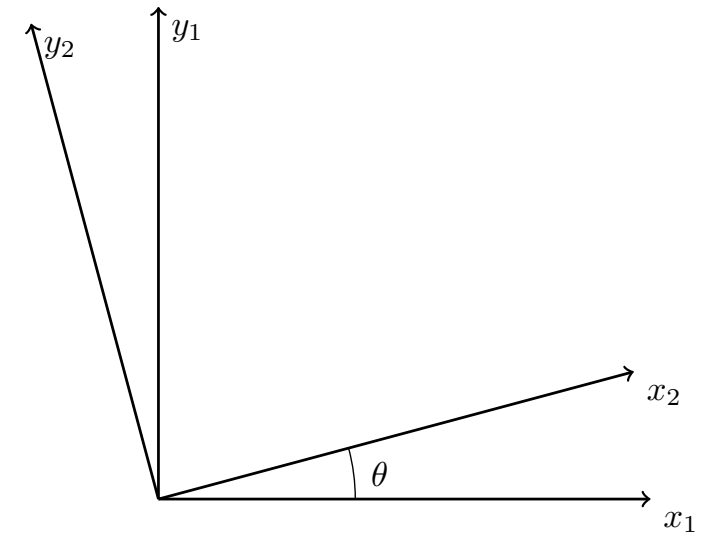

Figure 2.2: Visualization of relative angular rotation as described through skew angle $\theta$

The first attempt at reconstructing rotation rates was done converting from a single skewed IMU to the body axis IMU. However during flight tests the skewed IMUs would experience partial saturation with one or more axes unreadable. Therefore the partially saturated skewed IMUs needed to be reconstructed before transformation to the body axis reference frame.

The first method attempted for taking a partially saturated skewed IMU and converting those values to the body axis reference frame used a brute force guess and check method. When the mathematical relationship was known and one or more unsaturated values of a skewed IMU were known, potential body axes rotation rates were guessed and iterated over. The potential body axis values were compared to real values by converting the guessed potential rotation rates from the body axis reference frame to the skewed IMU. If the values matched the known recorded values from the skewed IMU, then it would be known that the guessed body axis values were also the real rotation rates of the body axis reference frame. For example, if IMU 1 was reporting two good values on the $x$ and $y$ channel and one nonsensical value on the $z$ channel, could the body axis rotation vector iterate over the $x, y$, and $z$ axes, such that searching for the set of body axes rotation rates, when transformed back to IMU 1, would match the two known values and produce the third unknown value on the $z$ channel. Exhaustive efforts showed a brute force method was computationally impractical as the processing time became unreasonable. Furthermore, the relationship between $x, y$, and $z$ channels were not unique. Meaning if two rotation rate channels were known, the third channel 
could have more than one value that solved the equations. The total number of options that solved the equations could reasonably be reduced by considering the most recent state of that saturated value and eliminating nonsensical options. The second and final method that was used to transform partially saturated skewed IMUs will be discussed in the next section where the mathematical relationship describing the reference frame transformation was further explored.

\subsection{Rotation Matrices}

The relationship between the skewed IMUs was defined through coordinate transformations. To navigate between skewed reference frames, rotational matrices were used to go between each coordinate frame. Coordinate transformations done for practical implementation are more easily visualized with Euler angles. Euler angles refer to a yawing orientation, a pitching orientation, and a rolling orientation. Defining the principle axis as yaw for the $z$ axis, pitch for the $y$ axis, and roll for the $x$ axis relates the Euler angles to the axis of motion. The use of Euler angles enables an intuitive understanding of the attitude change the body experiences.

Extending Figure 2.2, the components $x_{1}$ and $y_{1}$ of the coordinate system can be rotated into another frame $x_{2}$ and $y_{2}$ by the rotation angle $\theta$. The process can be applied to a three dimensional component by increasing the rotation matrix to a $3 \times 3$ shown in equations $2.1,2.2$, and 2.3. The result produced is the three component vector being rotated about one axis. In order to traverse the solution space required by the skewed IMUs, the rotation matrix has to be applied three times. That is to represent the three different changes in motion. First about the yaw axis, second about the pitch axis, and finally about the roll axis. Done independently, the three movements can be linked together by taking the result from the first rotation matrix and using that in the next rotation matrix, etc. Or, all three rotation matrices can be multiplied together beforehand and the original vector passed through the combined three dimensional rotation matrix shown by equation 2.4. In either case, the order is paramount. Similar convention must be used throughout the process as multiplying matrices are not communicative. The order used specifically for the research was to rotate the vector first about the yaw axis, second about the pitch axis, and finally about the roll axis. That order was used to go from the common body axis reference frame to the skewed IMU reference frame. In order to reverse the process and go from the frame of reference of the skewed IMU to the common body axis, the matrices had to be multiplied in reverse order. The transpose of the 
three dimensional rotational matrix begot the same outcome as reversing the order of multiplication.

$$
\begin{aligned}
& R_{z} \alpha=\left[\begin{array}{ccc}
\cos \alpha & -\sin \alpha & 0 \\
\sin \alpha & \cos \alpha & 0 \\
0 & 0 & 1
\end{array}\right] \\
& R_{y} \beta=\left[\begin{array}{ccc}
\cos \beta & 0 & \sin \beta \\
0 & 1 & 0 \\
-\sin \beta & 0 & \cos \beta
\end{array}\right] \\
& R_{x} \gamma=\left[\begin{array}{ccc}
1 & 0 & 0 \\
0 & \cos \gamma & -\sin \gamma \\
0 & \sin \gamma & \cos \gamma
\end{array}\right] \\
& R_{z y x}=\left[\begin{array}{ccc}
\cos \alpha \cos \beta & \cos \alpha \sin \beta \sin \gamma-\sin \alpha \cos \cos \gamma & \cos \alpha \sin \beta \cos \gamma+\sin \alpha \sin \gamma \\
\sin \alpha \cos \beta & \sin \alpha \sin \beta \sin \gamma+\cos \alpha \cos \cos \gamma & \sin \alpha \sin \beta \cos \gamma-\cos \alpha \sin \gamma \\
-\sin \beta & \cos \beta \sin \gamma & \cos \beta \cos \gamma
\end{array}\right] \\
& {\left[\begin{array}{ccc}
a_{11} & a_{12} & a_{13} \\
a_{21} & a_{22} & a_{23} \\
a_{31} & a_{32} & a_{33}
\end{array}\right]\left\{\begin{array}{l}
X_{b a} \\
Y_{b a} \\
Z_{b a}
\end{array}\right\}=\left[\begin{array}{c}
X 1_{\text {local }} \\
Y 1_{\text {local }} \\
Z 1_{\text {local }}
\end{array}\right]} \\
& {\left[\begin{array}{lll}
b_{11} & b_{12} & b_{13} \\
b_{21} & b_{22} & b_{23} \\
b_{31} & b_{32} & b_{33}
\end{array}\right]\left\{\begin{array}{l}
X_{b a} \\
Y_{b a} \\
Z_{b a}
\end{array}\right\}=\left[\begin{array}{c}
X 2_{\text {local }} \\
Y 2_{\text {local }} \\
Z 2_{\text {local }}
\end{array}\right]} \\
& {\left[\begin{array}{ccc}
c_{11} & c_{12} & c_{13} \\
c_{21} & c_{22} & c_{23} \\
c_{31} & c_{32} & c_{33}
\end{array}\right]\left\{\begin{array}{l}
X_{b a} \\
Y_{b a} \\
Z_{b a}
\end{array}\right\}=\left[\begin{array}{c}
X 3_{\text {local }} \\
Y 3_{\text {local }} \\
Z 3_{\text {local }}
\end{array}\right]}
\end{aligned}
$$

In order to extend the dynamic range of the MEMS gyroscope past the saturation limit, an augmented rotation matrix method was developed. Previously the coordinate transformations were done between a single IMU and the body reference frame shown in equations 2.5, 2.6, and 2.7. However it was after attempting the saturated value recovery, explained in the prior section, that the mathematical relationship was further leveraged to reconstruct saturated values. Even when 


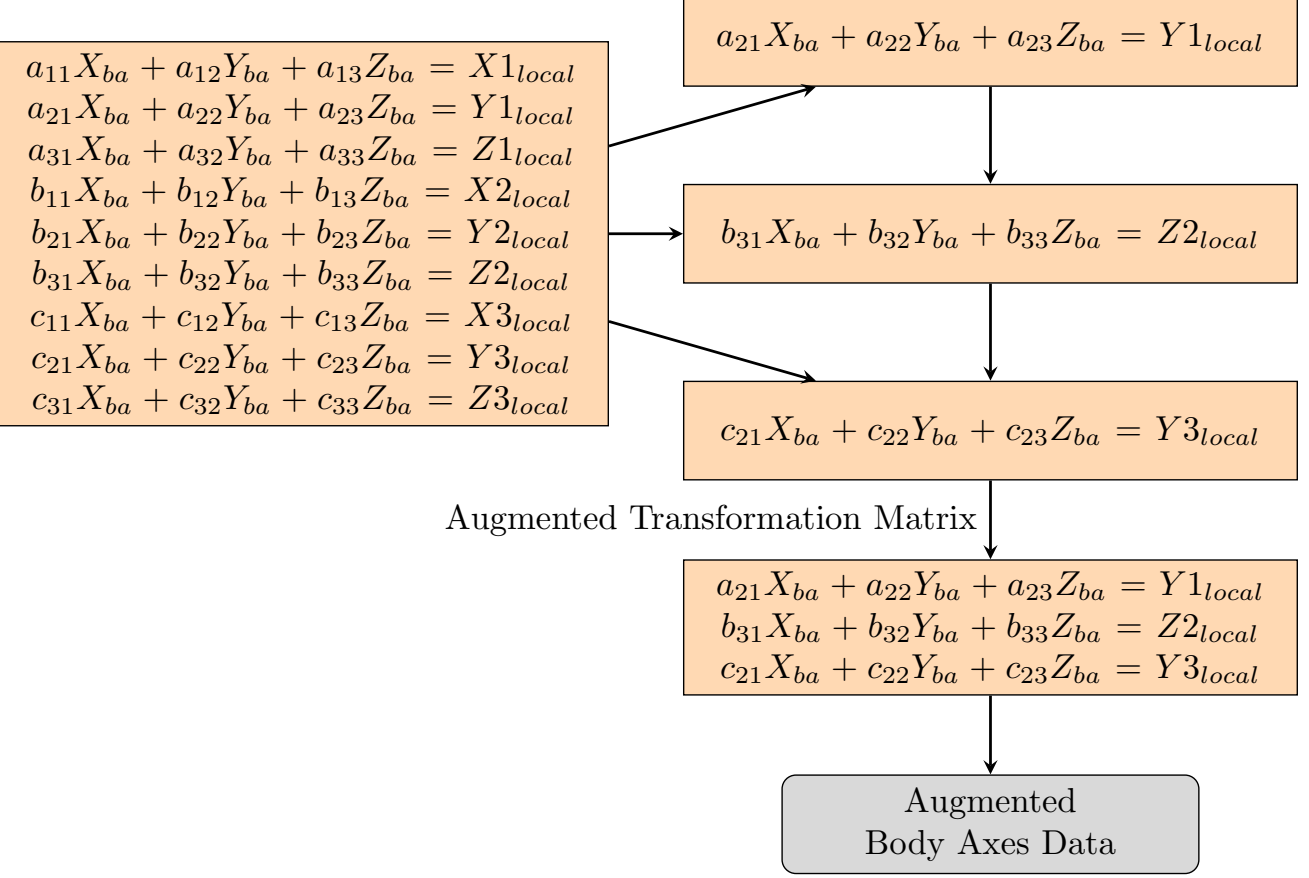

Figure 2.3: Augmented Matrix reconstruction for reference frame transformation

the skewed IMUs are partially saturated, the body axes rotation rates can be reconstructed. When the flying vehicle enters a period of saturated rotation rates on the body axis, there should be unsaturated values on the skewed IMUs. The aggregate system of equations for the three skewed IMUs, which can be seen in Figure 2.3, need only three unsaturated channels from the total of nine rotation rate channels. The IMUs from which the unsaturated values come from do not matter. For example, The $x$ channel for IMU 1, IMU 2, and IMU 3 could be used. Or the $x$ channel from IMU 1 , the $y$ channel from IMU 2, and the $z$ channel from IMU 3 could be used to create an augmented rotation matrix. With the augmented rotation matrix, the data could then be converted back to the body axis.

\subsection{Genetic Algorithm Skew Angles}

The angles between the skewed IMUs were the main factor in determining the ability of the system to record data above the saturation limit of the common body axis IMU. Therefore, exploring the combinations of offset angles for each yaw, pitch, and roll axis of each individual IMU was paramount. The goal was to find a set of offset angles such that they maximized the probability of the skewed IMU to record data that would otherwise be saturated on the principle body axes. As shown by the rotation matrices $2.1,2.2$, and 2.3 , the trigonometric components make the relationship inherently 
nonlinear. The solution space for the skew angles could therefore not be easily navigated.

In order to search the non-linear solution space of the skew angles, an evolutionary based search algorithm was used. Specifically, MATLAB's implementation of a genetic algorithm. In the research performed, the genetic algorithm was applied as a single and multi-objective approach. The yaw, pitch, and roll axes of each IMU were minimized as a function of the skew angles. The objective for the research was to use each one of the skewed IMUs to increase the probability of capturing one of the common body reference frame IMU's yaw, pitch, or roll rates during an instance of saturated data. That is to say, IMU 1 was tasked to back up the data on the yaw axis of the common reference frame, IMU 2 was tasked to back up the data on the pitch axis of the common reference frame, and finally IMU 3 was tasked to backup the data on the roll axis of the common reference frame IMU aligned with the body axis.

The genetic algorithm was applied as a single objective cost function. The cost function for the single objective case was to minimize the magnitude of the angular rotation rate of a single axes. Recall, part of the method was to use a skewed IMU to backup a single channel on the body axes. The cost function for the multi-objective algorithm was to minimize the magnitude of the angular rotation rate vector for all three axes on the skewed IMUs. The solution for the multi-objective case did not directly contribute to the skew angles that were determined. The purpose of using a multi-objective case was to represent the total magnitude that the skewed IMU would experience and to understand what the remaining two axes would experience when they were configured to provide redundancy for a single body axis channel.

The single objective function case was used as the primary tool to explore the solution space. The function was developed to mimic the real application of converting IMU data between reference frames. The genetic algorithm used artificially created data made to represent the body axes. The data created was random and uniformly distributed between the upper and lower bounds. The goal was to achieve reconstructed rates approximately double the saturation limit of the sensor. The artificial data was therefore bounded between negative four thousand and positive four thousand degrees per second. That enforced fifty percent of the data to be at least partially saturated from the perspective of the body axes. Prior to using a uniform distribution of random data, alternatively constructed data was considered. The data the genetic algorithm was trained on would ultimately determine the behavior of the skewed system during testing. If behavior of the flying body was well known, then the artificially created data could emulate that. Tailoring the data could benefit the system by being more capable for a certain scenario. For example, the created data could be modeled with higher or lower limits if that is what the case was believed to be for the real flying vehicle. 
The data could also be structured in a Gaussian manner with a higher probability distribution around the mean, which was zero degrees per second in the case of the research performed. It would seem reasonable to postulate the flying vehicle behavior operates more frequently in the unsaturated regime and the higher saturated values are less likely and therefore choose a Gaussian distribution for the data created for the genetic algorithm. However using a uniformly distributed data set over the given bounds was believed to make the skewed system more robust in reconstructing saturated values. The nature of the flying vehicle was aerodynamically unstable therefore creating data uniformly was believed to be the best option. By not manufacturing in potential biases, the system would be open to random and unforeseen behavior by the flying vehicle. The artificial body axis data would then be converted to a skewed IMU reference frame. The cost function would minimize the magnitude of the $x, y$, or $z$ channel while the genetic algorithm would search through the skew angle solution space; zero to ninety degrees. The genetic algorithm would perform a specified number of iterations and stop when the cost function improvement reduced.

To verify the plausibility of the designed offset angles that were selected from the results of the genetic algorithm, a statistical inquiry was performed in order to ensure the skewed IMUs had a statistically significant chance of recovering saturated data. Figure 2.4 shows the probability of recovering a minimum of three channels on the skewed IMUs below the rotational rate of two thousand degrees per second. The inquiry was done with the same style of random data used in the genetic algorithm, random numbers between negative four thousand and positive four thousand degrees per second.

Additional inquiry was done into the specifics of the saturated data. For example, the number of instances of certain saturation cases were noted and compared against how many of those specific cases were recoverable. When the body reference frame had one saturated value, those values were compared to the total number of data points to determine the prevalence in the total set of data points. The same was done for cases of two and three saturated values on the body reference frame and noted how many times those cases occurred. The probability of recovery for that specific case was then performed. Figure 2.5 shows the probability of recovering body reference frame data when the specific cases of one, two, or three saturated values on the body reference frame occurred. Further discussion on these results are in section 3.5 Discussion 


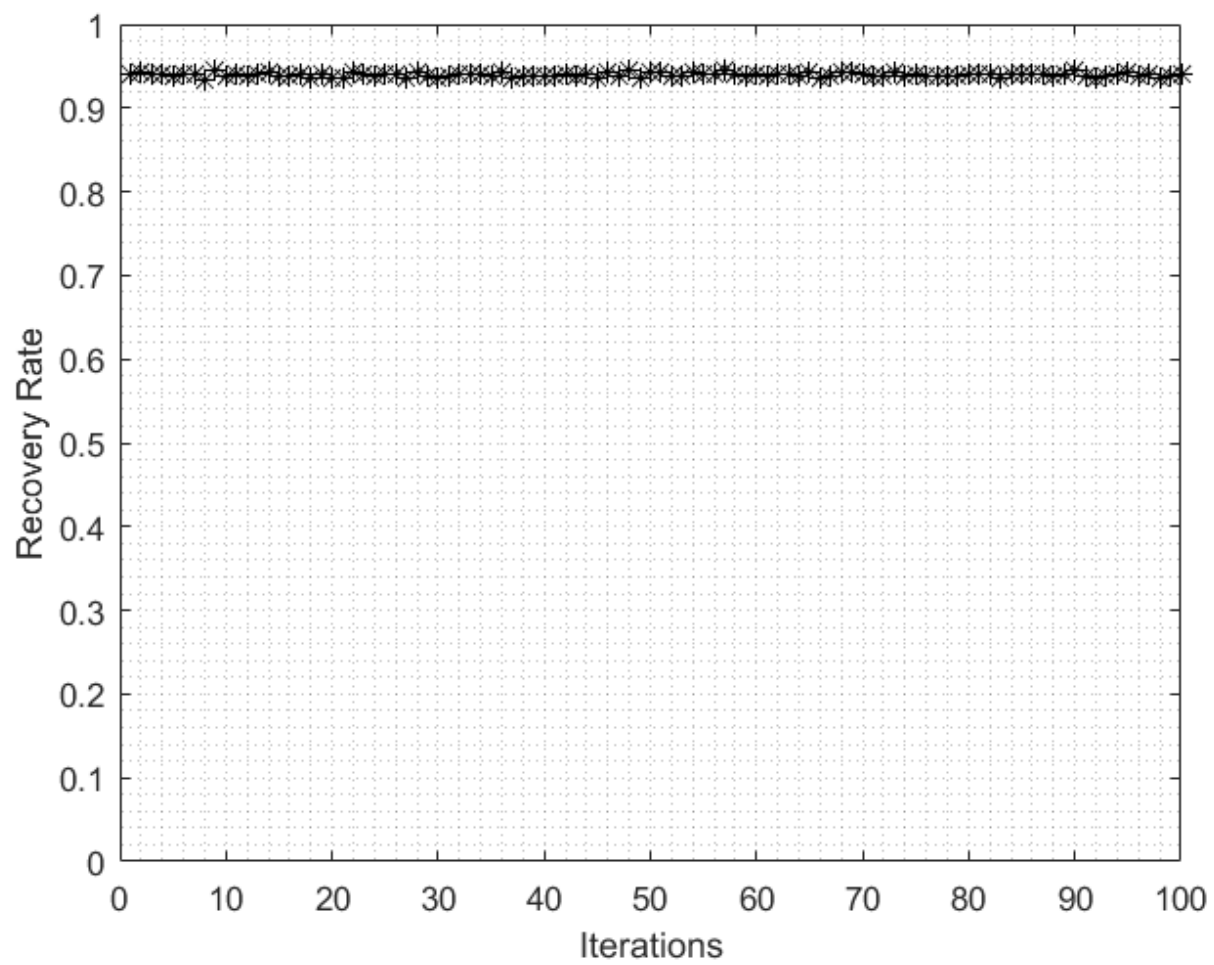

Figure 2.4: Monte Carlo simulation noting probability of recovering saturated rotation rates for the selected set of skew angles.

The Monte Carlo simulation was performed with ten thousand samples per iteration. A total of one hundred iterations were performed. For each sample, data was a random uniform distribution between negative four thousand and positive four thousand degrees per second. Each sample represented a data capture event with $x, y$, and $z$ channels. The artificial data created were from the body axes perspective. The body reference frame data was transformed to each of the three skewed IMU orientations using rotation matrices. The transformed data represented what the skewed IMUs would be experiencing from their perspective and were noted as the 'reported' data. The skewed IMU data were modified to represent what the logged data would actually look like. That required each value for a skewed IMU that was over the saturation limit was set to two thousand degrees per second. The recovery rate was then calculated by examining each sample step. A minimum number of three out of the nine channels were required to be unsaturated. With three unsaturated channels per sample, the rotational matrix equations could be solved to complete the transformation. The total number of recoverable instances per sample step was summed and divided by the total number of samples to calculate the percent recovery per iteration. Figure 2.6 displays the flow process for determining the possible recovery rate for the skewed configuration. 


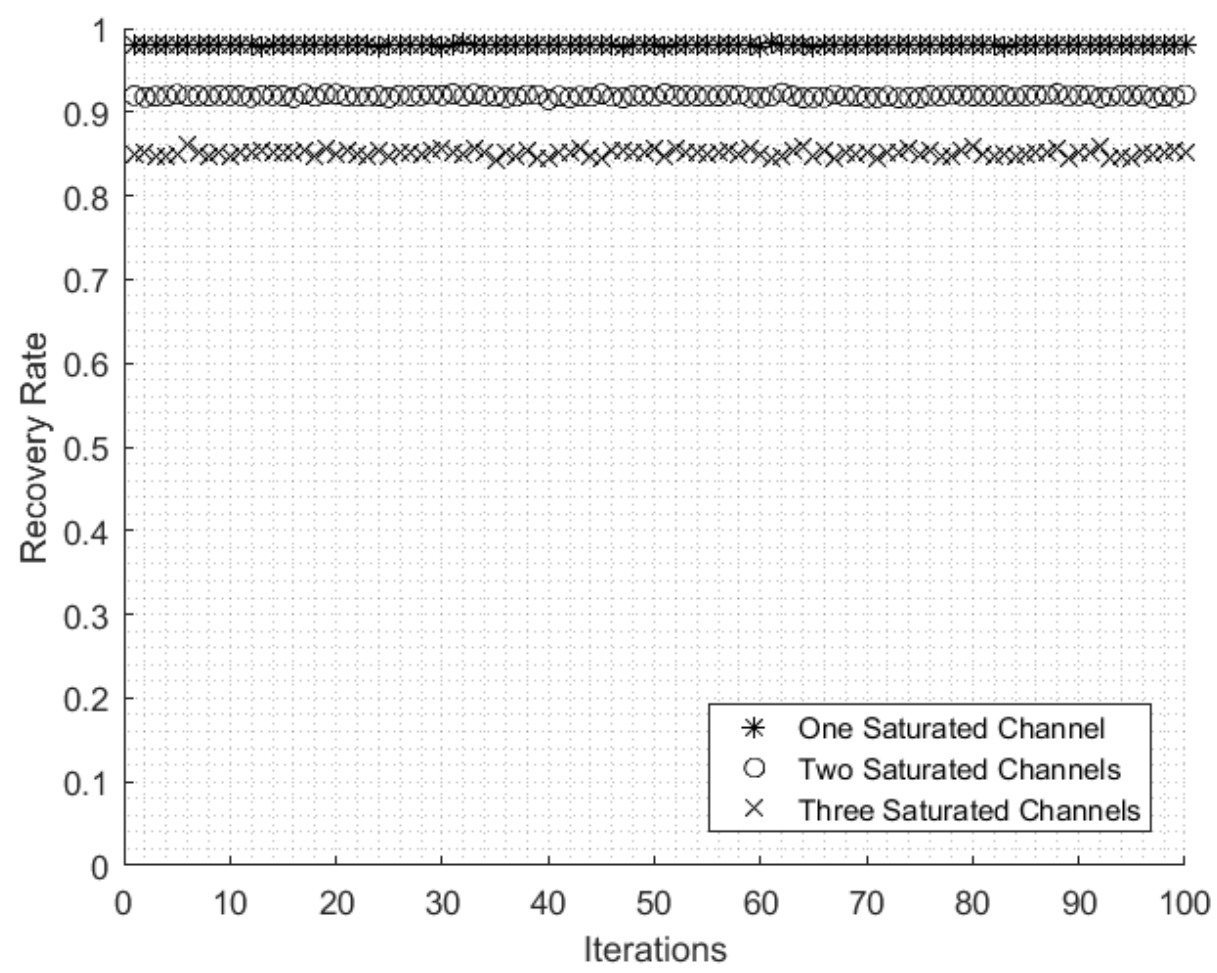

Figure 2.5: Monte Carlo simulation noting probability of recovering saturated rotation rates for cases of one saturated channel, two saturated channels, and three saturated channels on the body axis 


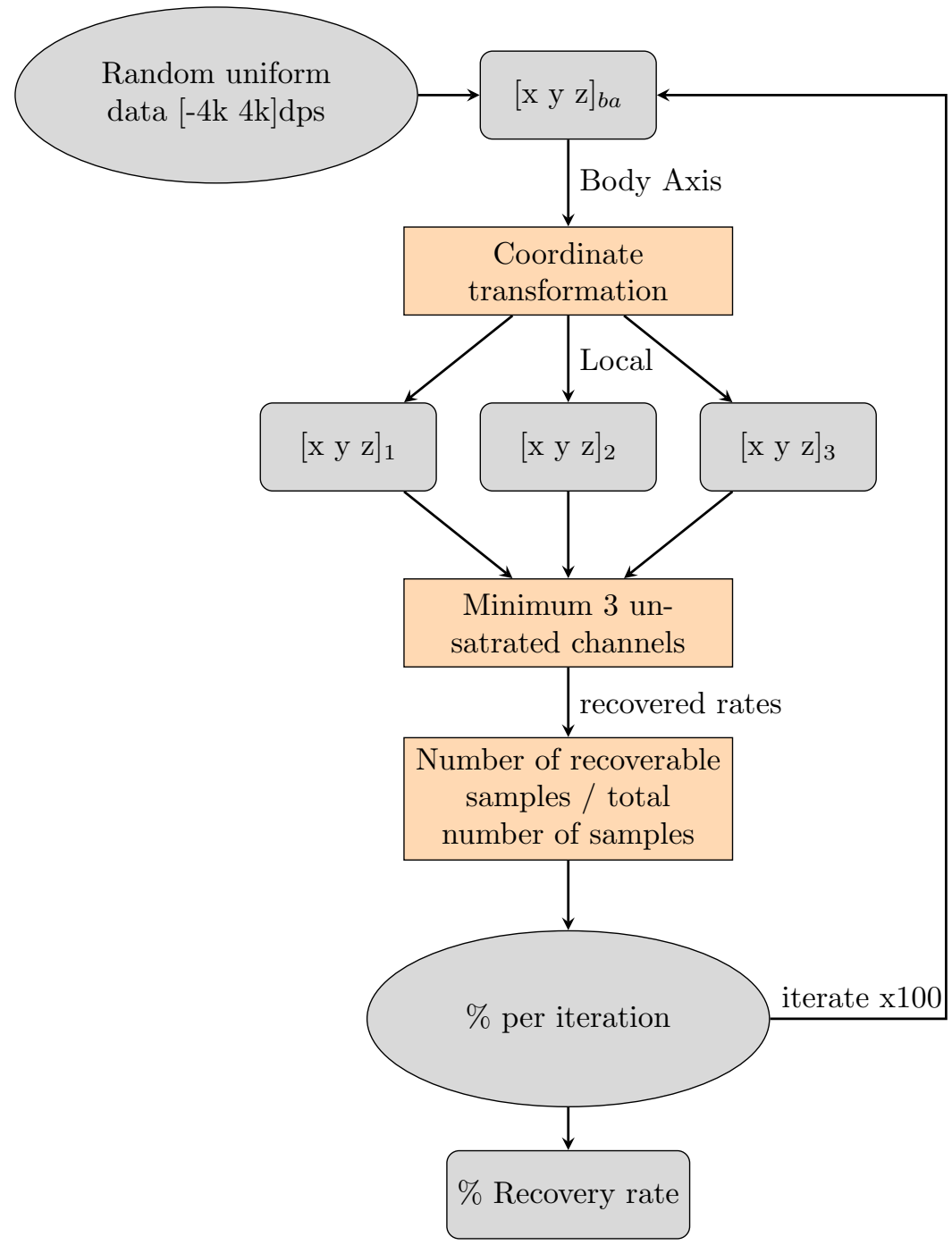

Figure 2.6: Monte Carlo recovery rate process 


\subsection{Flight Test Payload}

The test bed for the skewed redundant IMU configuration for the physical flying vehicle was highly constrained by the geometric and mass properties. Therefore the center of gravity and moments of inertia were critical. Adding instrumentation to the flight vehicle had to be carefully considered. The overall center of gravity for the flying vehicle (not shown) was noted and superposed to the internal cavity with which the instrumentation payload would be placed. A cap on the flying vehicle was removable and the IMU payload could be taken in and out. The structure of the holder was SLA 3D printed and the density of the resin was taken into consideration in designing the payload structure for center of gravity calculations. The outer dimensions of the sensor payload were bounded by the inner dimensions of the flying vehicle. Figure 2.7 shows initial attempts to place IMU boards inside the confined geometry of the holder. The holder had to be designed in two pieces due to the manufacturing limits of the build volume of the $3 \mathrm{D}$ printer.

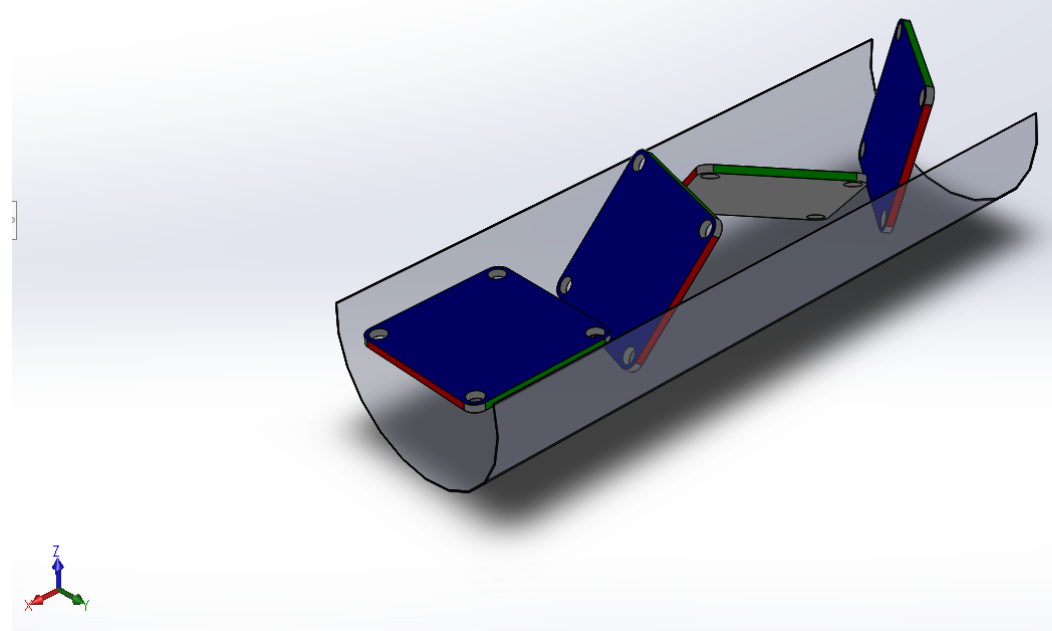

Figure 2.7: Initial effort to place skewed IMU platforms within internal geometry constraints

Figure 2.8 shows IMU sensor board inside the left portion of the holder. The right side of the image is where the battery was stored and the indentations were used for indexing the payload. After placement of the sensors, the center of gravity was significantly off from the target value. Weight ballasts had to be designed in order to shift the center of gravity back towards the end of the holder with the battery. The weight ballasts were fabricated from one eighth inch tungsten rods. Figure 2.9 shows the IMUs implemented in the 3D printed holder. 


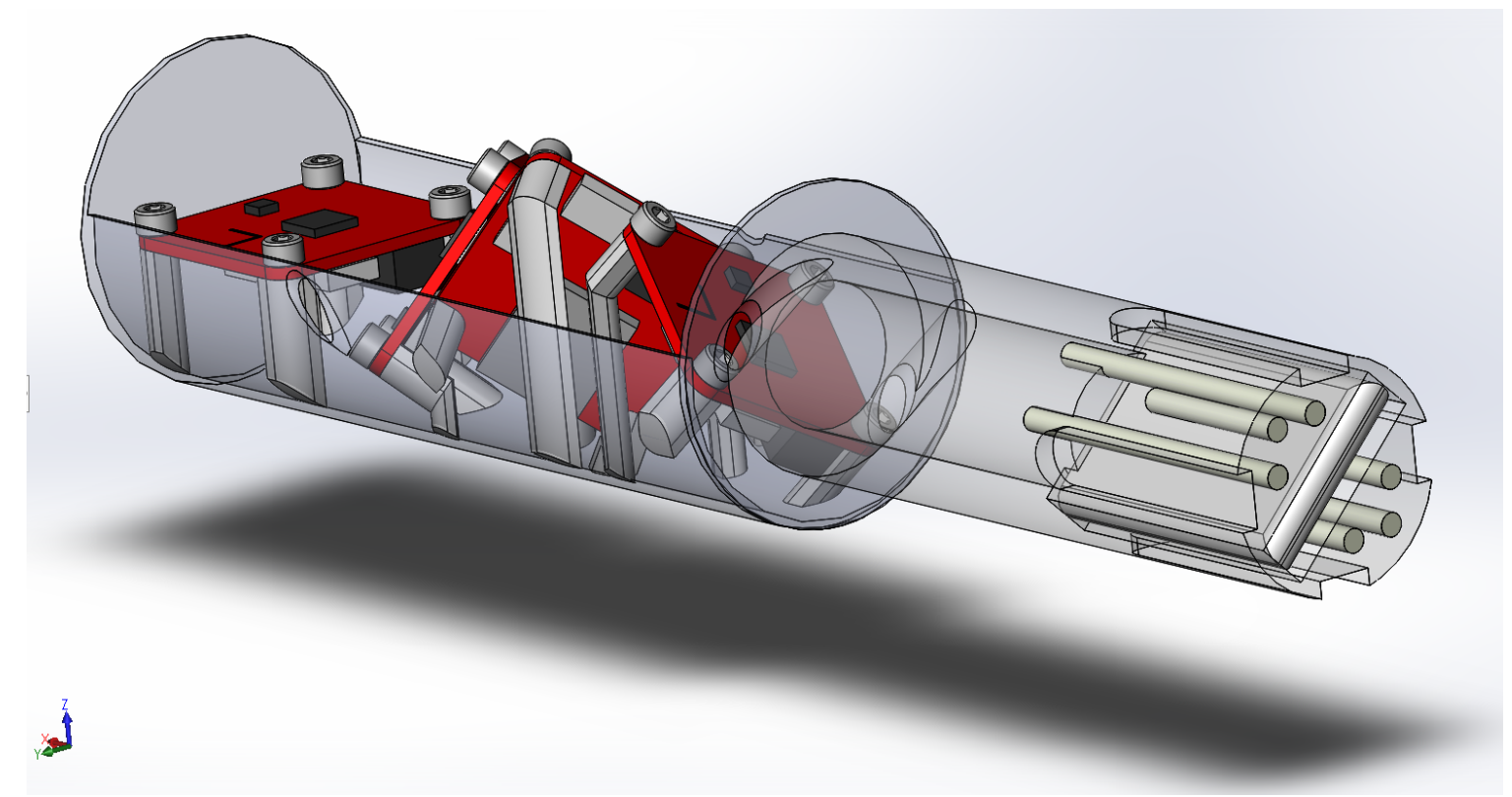

Figure 2.8: Instrumentation payload showing tungsten ballasts and battery pack

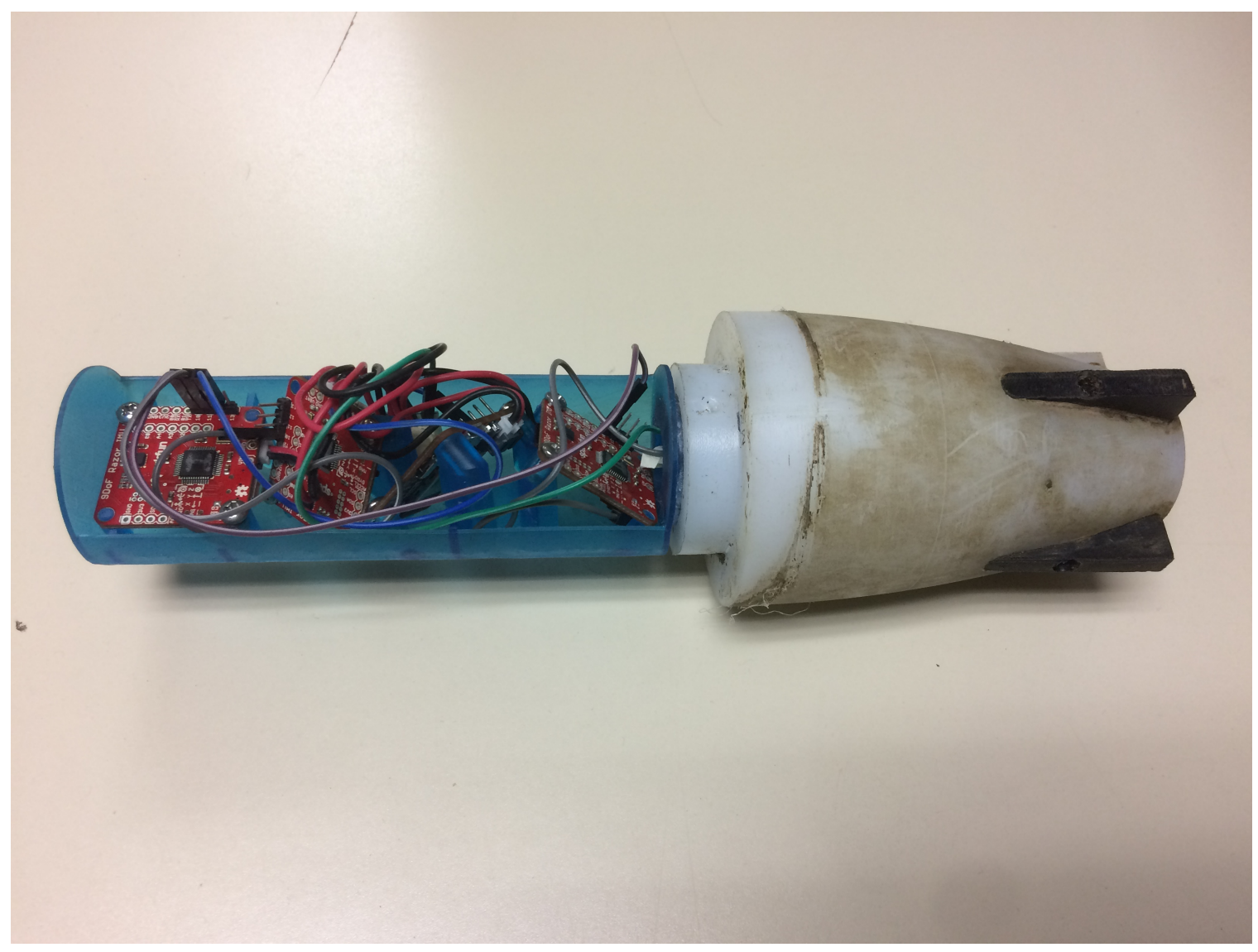

Figure 2.9: Instrumentation payload with IMUs wired together and placed in the tail cone of the flight vehicle 


\subsection{Wind Tunnel Test Payload}

The SparkFun Razor IMU was modeled as a solid part using a dial caliper with a precision of plus/minus 0.001 inches as dictated by the uncertainty of the caliper. The holder for the four IMUs were designed in the CAD software suite SolidWorks shown in Figure 2.10. The axes in SolidWorks were corrected to represent the 'right hand rule' as that is the notation of the IMUs. The IMU holder was designed as an assembly part that consisted of the IMUs and the holder structure that would be manufactured. The IMU that was to be aligned with the body axes of the UAV was placed in first, in continuity with the global coordinate system that was corrected as stated above. Each subsequent IMU was placed into the CAD environment and had their local coordinate system rotated as dictated by the skewed offset angles that were chosen and tabulated in section 3.1. The local rotation of each IMU axis was important in that it had to match the sequence in how the algorithm implemented the transformation mathematics. During the experiment, the rotations were done in the 'PQR' sequence or the yaw, pitch, and roll sequence. Once all four IMUs were placed into the assembly environment in CAD, the skewed IMUs were positioned precisely. Their location was chosen to create a physical layout that was compact and practical. It was necessary to mind the location of the on/off switch, power port, IO pins, and SD card so during implementation all IMUs could easily be operated. In addition, the supporting structure was designed to hold each IMU with as much rigidity as practical. Each IMU had four screw holes that were used to fasten them down. Specifically, two diagonal holes were used as indexers and the remaining two diagonal holes were used to screw down the IMU to the holder. The holder structure was designed so that the indexing holes had material that extruded past that of the resting surface and protruded through the indexing holes. As one would expect, the precision of the IMUs being aligned with the skew angles that were designed was paramount. Later, a sensitivity analysis was performed in order to understand the uncertainty introduced and how that manifested itself as the measured values passed through the algorithm. 


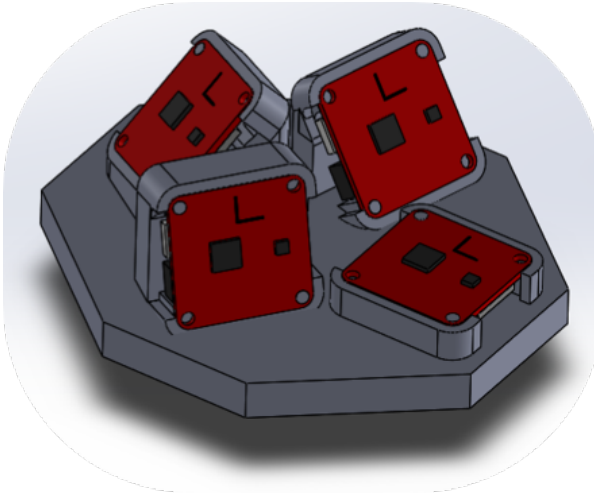

(a) CAD model of IMU holder Assembly

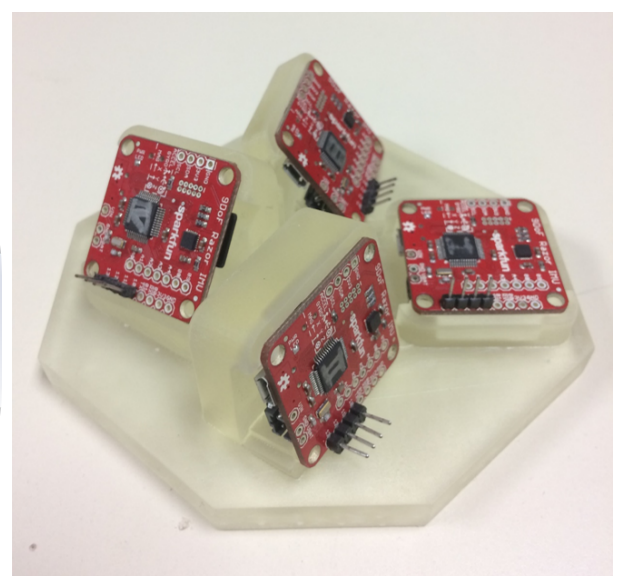

(b) 3d printed IMU holder assembly

Figure 2.10: IMU sensor holder

\subsection{Vicon Motion Capture}

The primary data acquisition system used was Vicon, a motion capture system with roots developed in the medical field and popularized in computer animated graphics. Vicon also has a strong presence in engineering applications. Vicon is an infrared camera system that provides motion tracking of objects within its field of view. The camera sees infrared light reflected by markers placed on a item of interest. Software is used to extend these capabilities into a meaningful application. The location of a marker can be determined when the Vicon cameras are spatially known relative to each other. When the Vicon system has knowledge of the camera locations relative to each other, it can compare the location of a marker and reconstruct it in virtual space. The cameras and software are calibrated by sweeping the test volume with an object with known geometrical properties between markers. Figure 2.11 shows a calibration wand. 


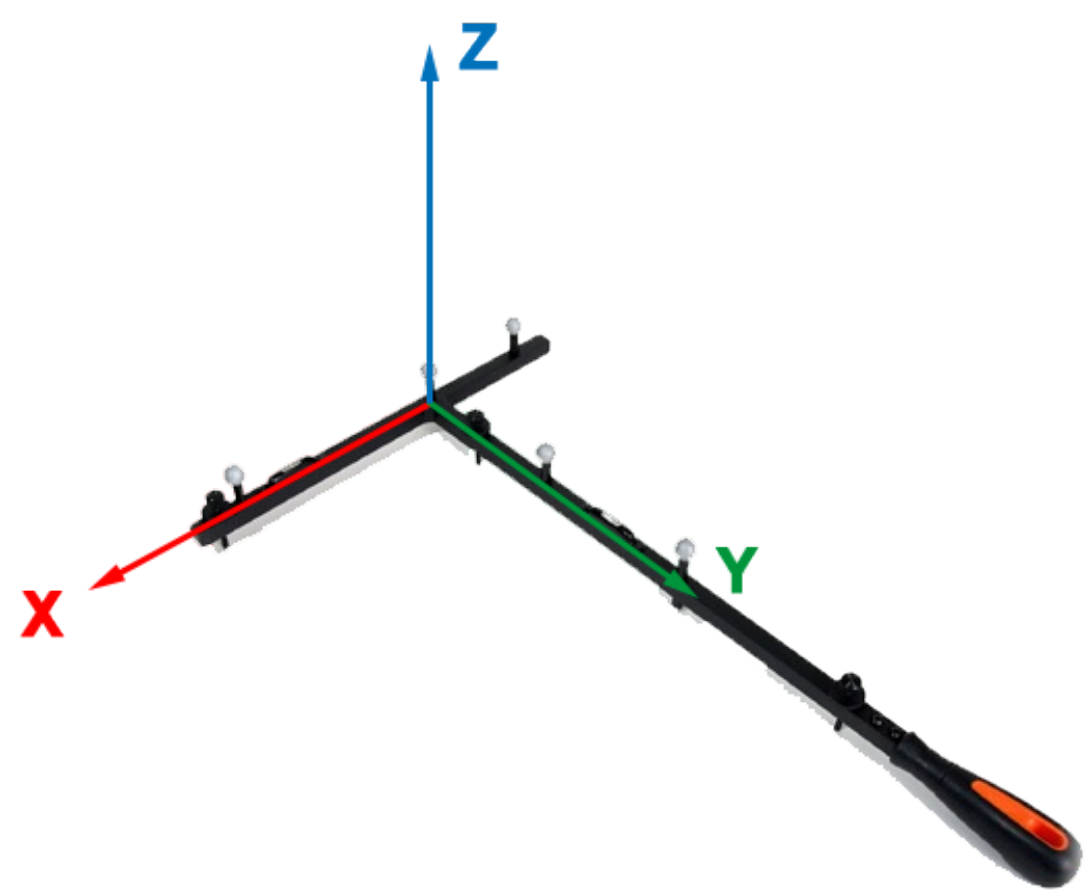

Figure 2.11: Vicon motion capture calibration wand. Photo: WVU-AJ Research Project

The markers on the wand create orthogonal lines that are used to represent the $x, y$, and $z$ axes and the planes they create. Once all the Vicon cameras are calibrated relative to each other, a local origin can be placed using the calibration wand which creates a coordinate reference frame within the software. The calibration calculations are done within the software. The software suite used for the research project was TRACKER which was created and maintained by Vicon. The next step was to create a model of the test specimen. The TRACKER software determines the orientation and subsequent behavior of an object by referencing the geometry of the model. The marker orientation and placement must be known physically in order to return meaningful data from the software. For example, when creating the model of the test specimen in the software, the markers were laid out to make sure the markers created orthogonal axes in the software. Additionally, the IMU labeled as the body axis IMU had to align with the axes created by the software in order to perform a comparison. Marker placement was paramount in maintaining alignment between the physical test specimen and the model in the software. It was necessary to ensure that the placement of subsequent markers did not create similarly spaced distances between markers. Figure 2.12 below shows the Euclidean distances between a proposed marker layout scheme and was used in Appendix A to show initial hand calculations performed to create an optimal marker placement layout. 


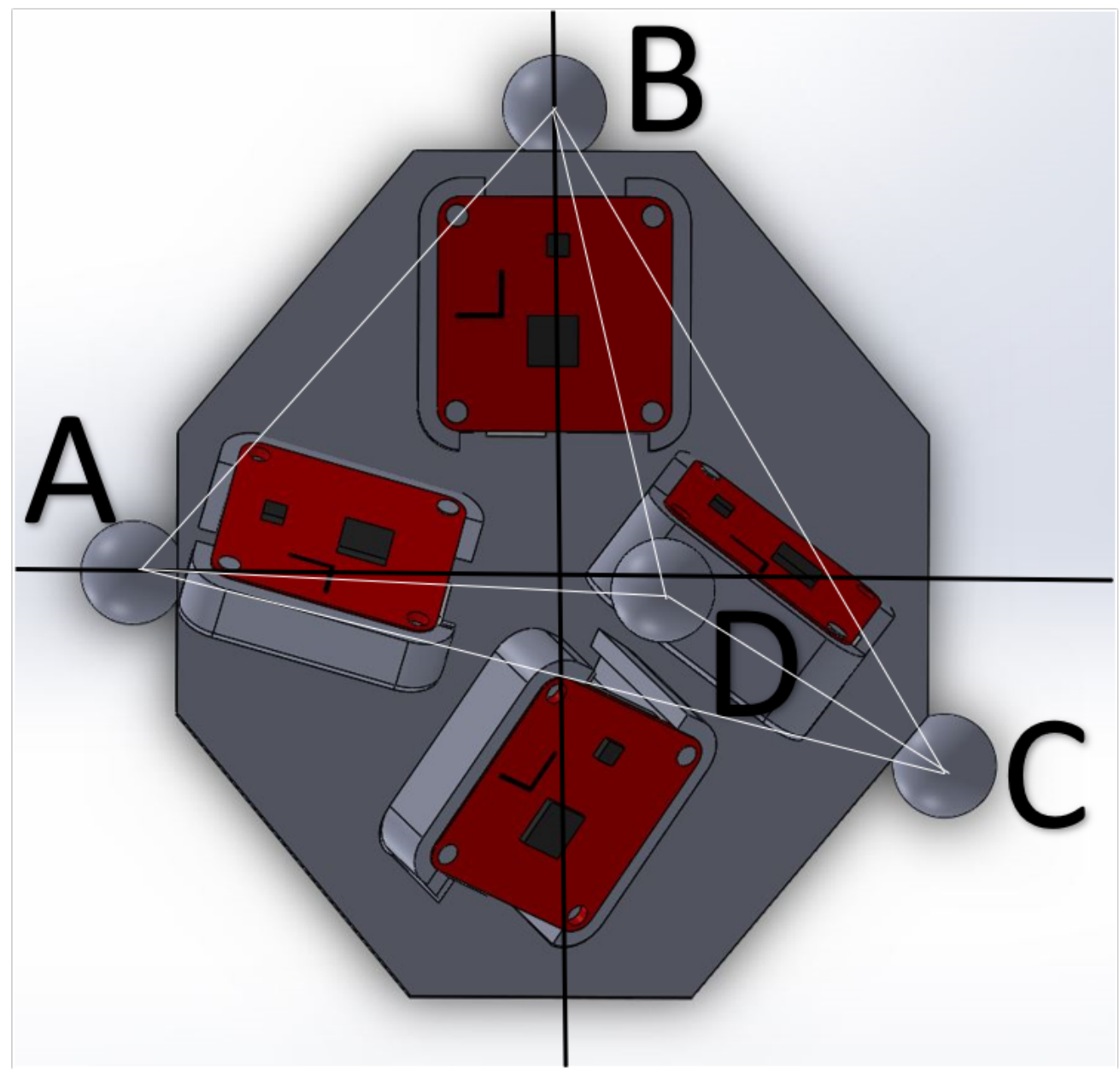

Figure 2.12: Example design of reflective Vicon marker placement establishing Euclidean distance between markers

The Vicon cameras were sensitive to the reflected infrared markers, so if an object utilized markers in a symmetric pattern, the software wouldn't be able to determine between the two possible mirror reflections that would occur with a symmetric marker pattern. Figure 2.13 shows an isometric view for the final configuration designed for marker placement. A fourth marker was placed underneath and to the rear of the test payload. The marker spheres used were $10 \mathrm{~mm}$ in diameter and had screw threads to easily take on or off the test payload. 


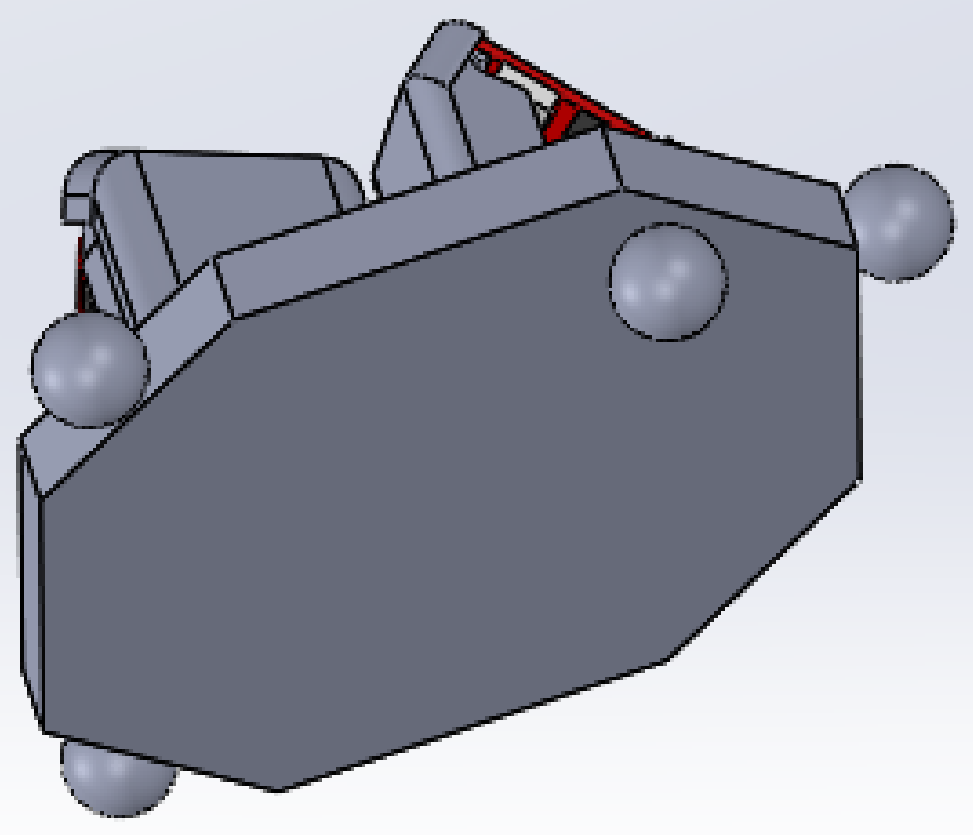

Figure 2.13: Final reflective marker configuration designed for the wind tunnel instrumentation payload 


\subsection{Test Setup}

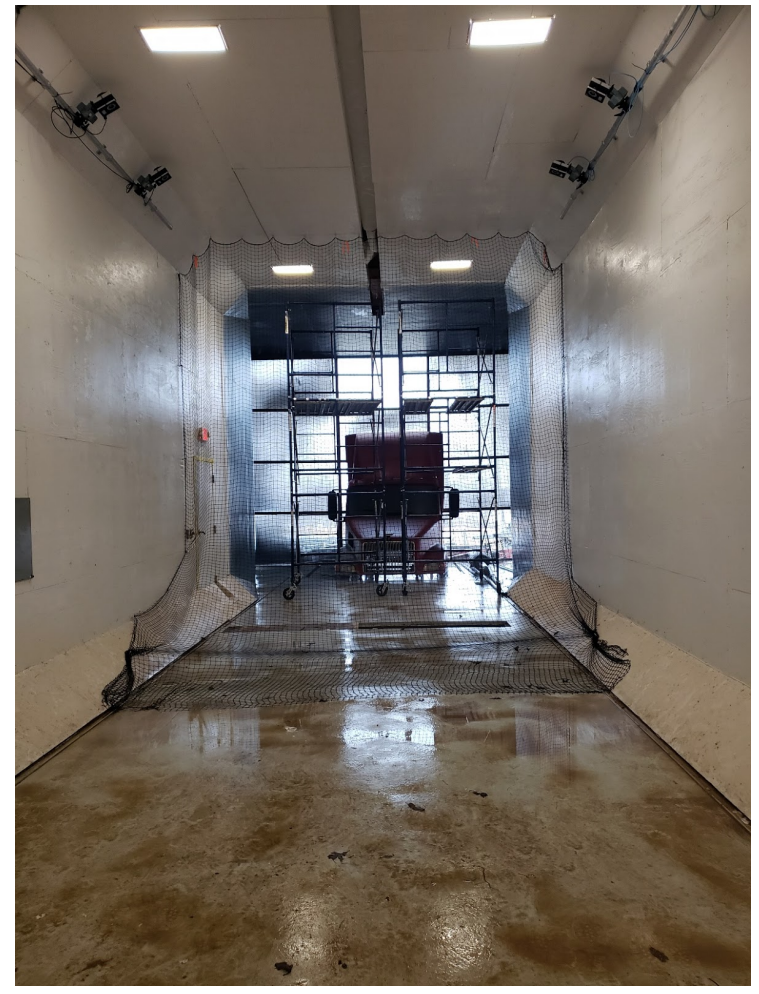

(a) Wind tunnel arresting net

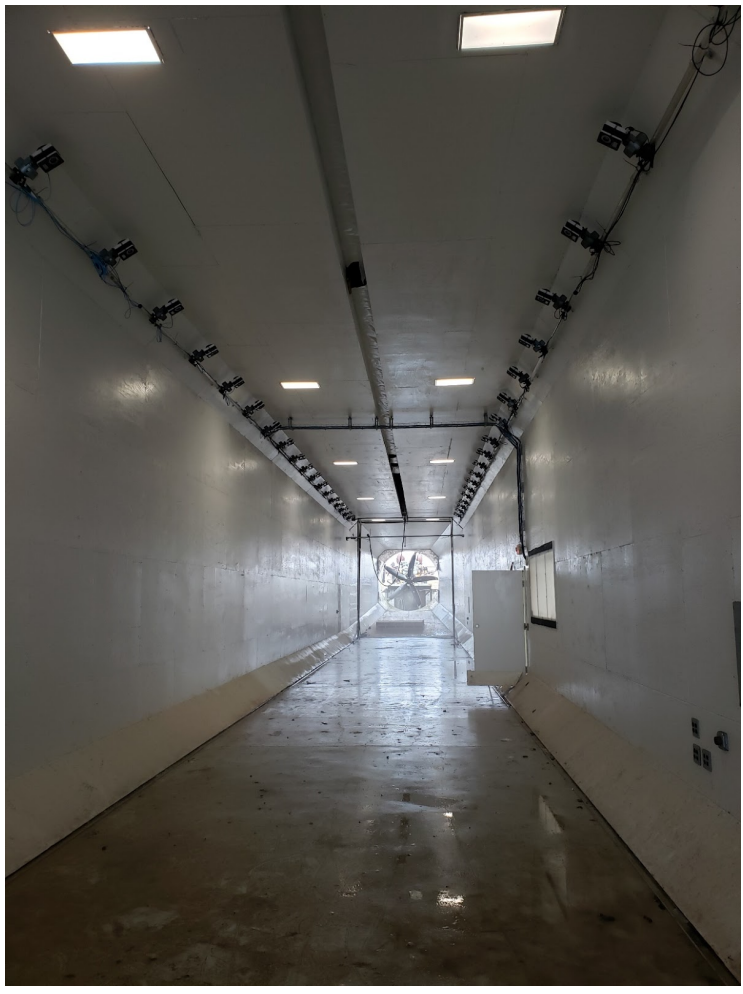

(b) Wind tunnel motion capture perspective

Figure 2.14: Reedsville, WV. Wind tunnel test setup

The experiment performed here measured the ability of the sensor configuration to recover saturated rotation rates before implementing the system in the flight test payload. The facility in which the experiment took place was the WVU wind tunnel in Reedsville, WV. The wind tunnel was approximately one hundred and twenty feet long. The cross section of the tunnel was approximately sixteen feet high and sixteen feet wide. The tunnel was an open return style tunnel with a tractor fan at the end of the tunnel. For the experiment performed, a model arrest system was constructed near the beginning of the entrance of the tunnel with the mindset to launch projectiles from midway or from farther down the tunnel. The arrest system was a net that was stretched across the cross section. Approximately two feet from the bottom of the floor, the net was sloped such that models would slide to the floor in a gentle manner so as to not damage the model that was launched. The data acquisition system was set up in the control room which sat at approximately halfway down the length of the tunnel.

The wind tunnel was set up for motion capture experiments with the Vicon cameras. The cameras were attached to metal poles that traversed the length of the tunnel at the top of each 


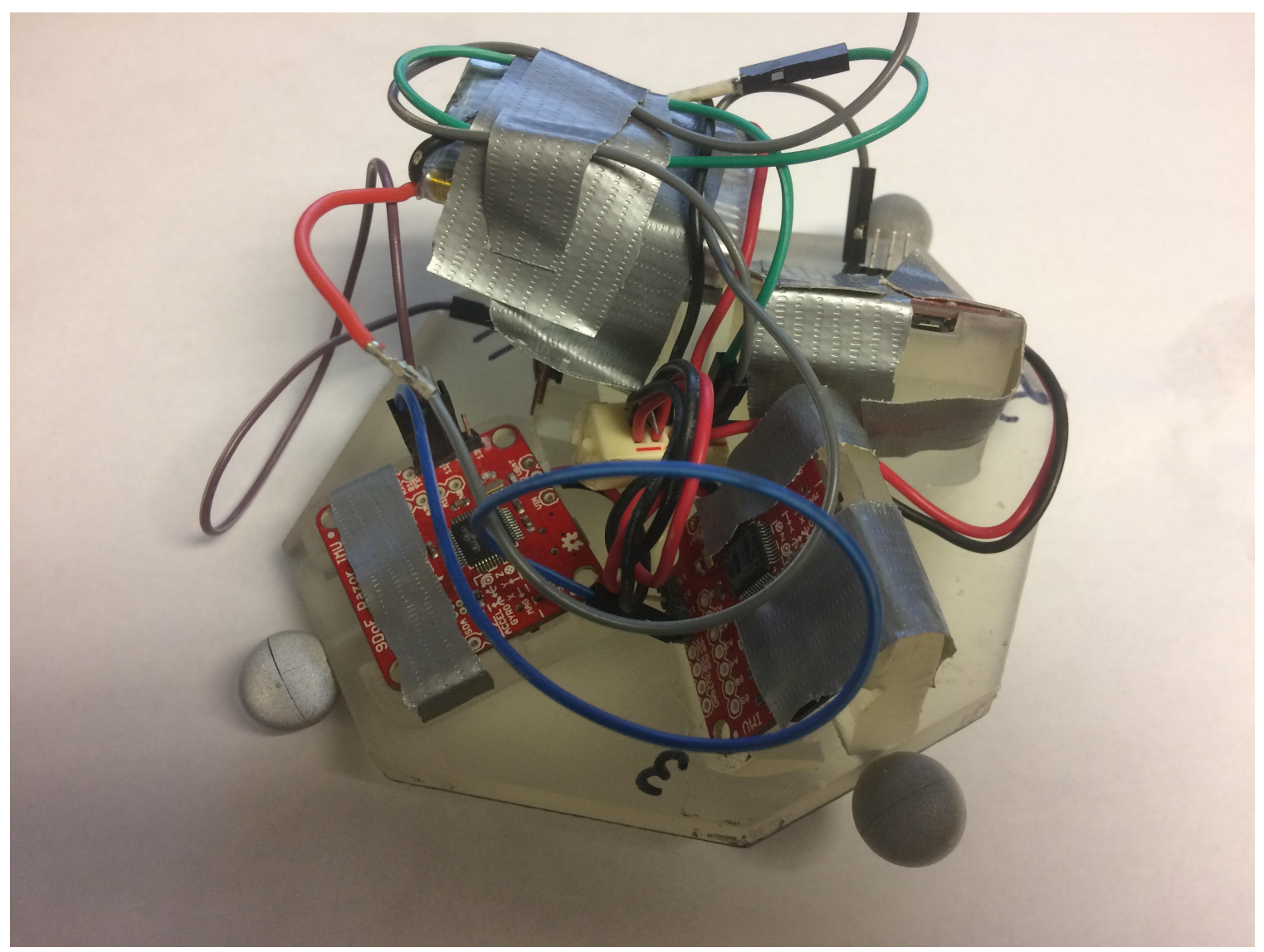

Figure 2.15: Wind tunnel instrumentation payload IMU holder wired for testing

wall. The motion capture cameras were spaced every six feet. The field of view was centered on the intersection of the floor and wall opposite of the camera. That is to say, the cameras along the top right of the tunnel looked at the bottom left of the tunnel. The intersection of cameras from the left and right side created a dense interrogation volume in the wind tunnel test section. TRACKER software was set to record at a sampling rate of one thousand hertz.

As an off the shelf component, the SparkFun Razor IMU was packaged as a plug-and-play sensor. The board came preloaded with firmware to operate the IMU and documentation for customization. Each IMU was configured to record accelerations, rotation rates, and the magnetometer was activated as it was used as part of the onboard firmware to correct gyroscopic drift bias. The IMUs were set to record data at their maximum sampling rate of one hundred hertz. The IMUs were synchronized temporally by the body axis aligned IMU configured as the primary and the skewed IMUs configured as replicates. Upon power being applied to the IMUs, a simple routine was activated that waited a sufficient amount of time to ensure each IMU was initiated. After waiting that time period, the master would send a start command to the slaves and which would start logging data. 
Once all systems were activated on the IMU, the motion capture system would be triggered from the control room. The IMU holder with its payload of the skewed IMUs was launched down the center of the wind tunnel into the arresting system. The goal for the experimental test was for the IMU holder to enter a region where the rotational rates on the body axis were close to double the saturation limit of the sensors. During that region, the body axis would be saturated on at least one if not all channels. The motion capture would record the true rotational rates of the IMU holder and would be used as verification against the recorded IMU data once it was passed through the algorithm. Figure 2.16 displays the typical test environment.

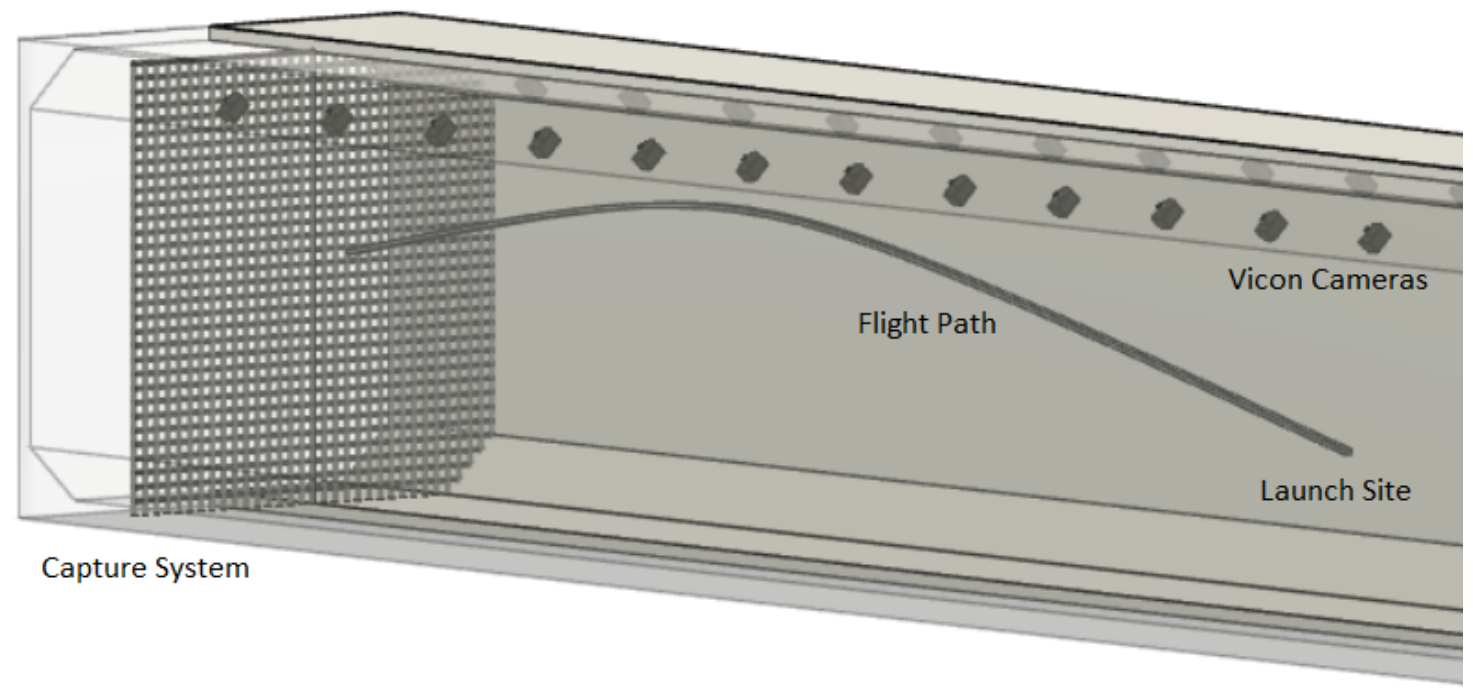

Figure 2.16: Wind tunnel illustration visualizing the arresting system on the left hand side, the motion capture cameras along the top, and a typical curved flight path of the test payload starting from right to left.

\section{$2.9 \quad$ Post-Processing Data}

The data from the IMUs were stored onboard the sensors using a microSD card. The data from the Vicon TRACKER software was taken from the wind tunnel instrumentation computer on a portable USB drive. The data from the separate systems were loaded onto a computer for where all the post 
processing data reduction was done in MATLAB.

In order to combine signals from four separate sensors together, a Kalman filter was used. Because a mathematical representation of the flight vehicle dynamics did not exist yet, recall the research project was trying to recreate the aerodynamic coefficients that described the physics of the flight vehicle, a simple method was used during the prediction step of the filter. Where normally a physics based model of the system would beget a sufficiently accurate prediction into the future step, a simple linear extrapolation into the future step was used for the prediction step of the filter. There were instances where the filter obviously lagged behind the updated results when there were sudden changes in motion, however the filter still performed sufficiently well. 


\section{Chapter 3}

\section{Results}

\subsection{Genetic Algorithm}

The genetic algorithm was used to extract skew angles that the IMU sensors were aligned with. Because a genetic algorithm is an optimization algorithm, there were multiple sets of angles that could have performed similarly, however the values that were selected were what the genetic algorithm converged upon and provided the best ability to extend the dynamic range of the sensors. Table 3.1 shows the skew angles calculated for each individual IMU by the genetic algorithm.

Table 3.1: IMU skew angles in degrees

\begin{tabular}{||c||ccc||}
\hline$\theta$ & $\mathrm{P}$ & $\mathrm{Q}$ & $\mathrm{R}$ \\
\hline IMU 1 & 35 & -30 & -20 \\
IMU 2 & 20 & 50 & 65 \\
IMU 3 & 65 & 50 & 20 \\
\hline
\end{tabular}

\subsection{Sensitivity Analysis}

A sensitivity analysis was performed to quantify the behavior of a unit measurement from input to output of the transformation algorithm. The analysis was organized to reflect the behavior of each individual rotational channel in an isolated manner as well as interaction between simultaneously active rotational channels.

Tables 3.2 through 3.4 represent an input on either the $x, y$, or $z$ channel respectfully. The input was analyzed from the body reference frame to show how a single unit rotation would propagate through the transformation matrices. As the rotation increases on the input, the subsequent values 
from the independent channels follows proportionally.

Table 3.2: X Axis Channel contribution

\begin{tabular}{||c||ccc||}
\hline BA & Axis & X & $\mathrm{Z}$ \\
IMU1 & 0.8138 & 0.2962 & -0.5000 \\
IMU2 & 0.2717 & -0.5826 & 0.7660 \\
IMU3 & 0.6040 & -0.2198 & 0.7660 \\
\hline
\end{tabular}

Table 3.3: Y Axis Channel contribution

\begin{tabular}{|c||ccc||}
\hline BA & Axis & $\mathrm{Y}$ & $\mathrm{Z}$ \\
$\mathrm{IMU} 1$ & 0 & 1 & 0 \\
$\mathrm{IMU} 2$ & 0.5497 & 0.6717 & -0.4967 \\
$\mathrm{IMU} 3$ & 0.9624 & 0.1597 & -0.2198 \\
\hline
\end{tabular}

Table 3.4: Z Axis Channel contribution

\begin{tabular}{|c||ccc||}
\hline AMU & $\mathrm{X}$ & $\mathrm{Y}$ & $\mathrm{Z}$ \\
\hline $\mathrm{BA}$ & 0 & 0 & 1 \\
IMU1 & 0.1887 & 0.6791 & 0.7094 \\
IMU2 & 0.0058 & 0.7969 & 0.6040 \\
IMU3 & 0.0058 & 0.9624 & 0.2717 \\
\hline
\end{tabular}

Tables 3.5 through 3.8 display results from the same process as described above. The four types of interactive combinations possible are tabulated. As the input increases, the output as noted by the IMUs increases proportional to the input. 
Table 3.5: X \& Y Axis Channel contribution

\begin{tabular}{||c||ccc||}
\hline IMU Axis & $\mathrm{X}$ & $\mathrm{Y}$ & $\mathrm{Z}$ \\
\hline BA & 1 & 1 & 0 \\
IMU1 & 0.2641 & 0.9679 & -0.9967 \\
IMU2 & 1.2340 & -0.4229 & 0.5462 \\
IMU3 & 1.4010 & -0.0602 & 0.1835 \\
\hline
\end{tabular}

Table 3.6: Y \& Z Axis Channel contribution

\begin{tabular}{||c||ccc||}
\hline IMU Axis & $\mathrm{X}$ & $\mathrm{Y}$ & $\mathrm{Z}$ \\
\hline BA & 0 & 1 & 1 \\
IMU1 & -0.3610 & 1.3507 & 0.2127 \\
IMU2 & 0.9681 & 0.9566 & 0.3842 \\
IMU3 & 0.8027 & 1.1221 & -0.3109 \\
\hline
\end{tabular}

Table 3.7: X \& Z Axis Channel contribution

\begin{tabular}{||c||ccc||}
\hline IMU Axis & $\mathrm{X}$ & $\mathrm{Y}$ & $\mathrm{Z}$ \\
\hline BA & 1 & 0 & 1 \\
IMU1 & 1.0025 & 0.9753 & 0.2094 \\
IMU2 & 0.2774 & 0.2144 & 1.3701 \\
IMU3 & 0.6098 & 0.7425 & 1.0377 \\
\hline
\end{tabular}

Table 3.8: X, Y, \& Z Axis Channel contribution

\begin{tabular}{||c||ccc||}
\hline IMU Axis & $\mathrm{X}$ & $\mathrm{Y}$ & $\mathrm{Z}$ \\
\hline BA & 1 & 1 & 1 \\
IMU1 & 0.4528 & 1.6469 & -0.2873 \\
IMU2 & 1.2398 & 0.3741 & 1.1502 \\
IMU3 & 1.4067 & 0.9022 & 0.4551 \\
\hline
\end{tabular}

\subsection{Uncertainty Analysis}

The uncertainty over the experiment was considered with two separate cases. Sources of uncertainty that influenced the experiment were found to be from the MPU-9250 sensor chip, and how the IMU was spatially oriented from the 3D printed resin holder. During post processing, time dilation was identified as well.

In the first case, an individual IMU would not be in a saturated state and would therefore be capturing all three axes of rotation. Uncertainty would be present from the sensor and from the skew angles from 3D printing. In the second case, an individual IMU wold be saturated on an axis and the algorithm would use channels from different IMUs, their time dilation with respect to each 
other would cause uncertainty to arise.

Uncertainty analysis was performed using the method called adding in quadrature for more than one variable. Equations 3.1 and 3.2 below show the method for multiplication and division for relative uncertainty $[21]$.

$$
\begin{gathered}
q=\frac{x \times \ldots \times z}{u \times \ldots \times w} \\
\frac{\delta q}{|q|}=\sqrt{\left(\frac{\delta x}{x}\right)^{2}+\ldots+\left(\frac{\delta z}{z}\right)^{2}+\left(\frac{\delta u}{u}\right)^{2}+\ldots+\left(\frac{\delta w}{w}\right)^{2}}
\end{gathered}
$$

For complicated expressions like trigonometric functions, the uncertainty can be propagated by taking the difference of the minimum and maximum value. Take the arbitrary example of $q=\cos \theta \pm \delta \theta$. It follows in equation 3.3 that the uncertainty can be found as shown.

$$
|\delta q|=|\cos (\theta+\delta \theta)-\cos (\theta-\delta \theta)|
$$

The first source of uncertainty calculated was the discrepancy in the IMU offset angle. The SLA printer, Formlabs 2 had a laser spot size of $140 \mu \mathrm{m}\left(\approx 0.006^{\prime \prime}\right)$ and layer thickness capability of $25-300 \mu \mathrm{m}\left(\approx 0.001-0.012^{\prime \prime}\right)$. The part was printed at $50 \mu \mathrm{m}$ layer thickness. To make a conservative calculation, $100 \mu \mathrm{m}$ or $0.004^{\prime \prime}$ layer thickness was used to calculate the skew angle uncertainty.

$$
\begin{gathered}
\delta \theta=\operatorname{atand}\left(\frac{y}{x}\right) \\
\delta \theta=\operatorname{atand}\left(\frac{0.008^{\prime \prime}}{1.0^{\prime \prime}}\right)=0.458^{\circ} \\
\delta \theta=0.5^{\circ}
\end{gathered}
$$

Figure 3.1 below shows a 2D cartoon illustrating how uncertainty arises through the 3D printed IMU holder. The horizontal line represents the intended posture of the IMU but due to the layer resolution uncertainty there is a possibility of having an unintended angle.

The uncertainty propagation through the experiment can largely be captured through the equations of relative motion. The yaw, pitch, and roll rotation matrix 2.4 from page 13 can be deconstructed into equations $3.7,3.8$, and 3.9 respectively. 


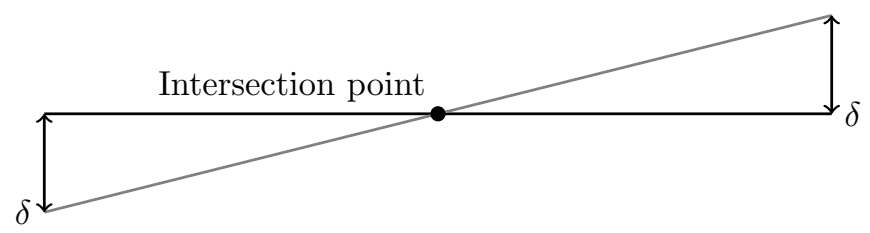

Figure 3.1: Cross section representation of 3D printed uncertainty due to layer thickness and the maximum difference possible

$$
\begin{gathered}
\cos (\alpha) \cos (\beta) X_{b a}+(\cos (\alpha) \sin (\beta) \sin (\gamma)-\sin (\alpha) \cos (\gamma)) Y_{b a} \\
+(\cos (\alpha) \sin (\beta) \cos (\gamma)+\sin (\alpha) \sin (\gamma)) Z_{b a}=X_{\text {local }} \\
\sin (\alpha) \cos (\beta)) X_{b a}+(\sin (\alpha) \sin (\beta) \sin (\gamma)+\cos (\alpha) \cos (\gamma)) Y_{b a} \\
+(\sin (\alpha) \sin (\beta) \cos (\gamma)-\cos (\alpha) \sin (\gamma)) Z_{b a}=Y_{\text {local }} \\
-\sin \beta X_{b a}+\cos \beta \sin \gamma Y_{b a}+\cos \beta \cos \gamma Z_{b a}=Z_{\text {local }}
\end{gathered}
$$

Equation 3.9 will be used to demonstrate the calculation of the IMU holder contribution. In the matrix form, these sine and cosine functions become constants seen on page 13. The relative uncertainty was calculated using the skew angles from IMU 1 where $\alpha=20, \beta=30, \gamma=-35$, and $\delta \theta=0.5$ degrees. The relative uncertainty for the IMU value is $\pm 3 \%$. The sine and cosine coefficient uncertainty is found in the absolute form using equation 3.3 and then converted to relative uncertainty.

$$
\begin{aligned}
-(0.5000 \pm 0.0151)\left(X_{b a} \pm 3 \%\right)+ & (0.866 \pm 0.0087)(-0.5736 \pm 0.0143)\left(Y_{b a} \pm 3 \%\right) \\
& +(0.866 \pm 0.0087)(0.8192 \pm 0.0100)\left(Z_{b a} \pm 3 \%\right)=Z_{l o c a l}
\end{aligned}
$$




$$
\begin{aligned}
-(0.5000 \pm 3 \%)\left(X_{b a} \pm 3 \%\right)+(0.866 \pm & 1 \%)(-0.5736 \pm 3 \%)\left(Y_{b a} \pm 3 \%\right) \\
& +(0.866 \pm 1 \%)(0.8192 \pm 1 \%)\left(Z_{b a} \pm 3 \%\right)=Z_{\text {local }}
\end{aligned}
$$

$$
-0.5 X_{b a} \pm 4 \%-0.4967 Y_{b a} \pm 4 \%+0.7094 Z_{b a} \pm 3 \%=Z_{l o c a l}
$$

Considering the remaining cases, the aggregate uncertainty for each channel of an IMU was $4 \%$.

Table 3.9: sine and cosine relative uncertainty

\begin{tabular}{||c||c|c||}
\hline IMU 1 & $\cos \theta$ & $\sin \theta$ \\
\hline$\alpha=20$ & $0.94 \pm 0.006$ & $0.34 \pm 0.02$ \\
$\beta=30$ & $0.87 \pm 0.009$ & $0.50 \pm 0.02$ \\
$\gamma=-35$ & $0.82 \pm 0.01$ & $-0.57 \pm 0.01$ \\
\hline \hline IMU 2 & $\cos \theta$ & $\sin \theta$ \\
\hline$\alpha=-65$ & $0.42 \pm 0.02$ & $-0.91 \pm 0.007$ \\
$\beta=-50$ & $0.64 \pm 0.01$ & $-0.77 \pm 0.01$ \\
$\gamma=-20$ & $0.94 \pm 0.006$ & $-0.34 \pm 0.02$ \\
\hline \hline IMU 3 & $\cos \theta$ & $\sin \theta$ \\
\hline$\alpha=-20$ & $0.94 \pm 0.006$ & $-0.34 \pm 0.02$ \\
$\beta=-50$ & $0.64 \pm 0.01$ & $-0.77 \pm 0.01$ \\
$\gamma=-65$ & $0.42 \pm 0.02$ & $-0.91 \pm 0.007$ \\
\hline
\end{tabular}

\subsection{Flight Test}

The data from each local IMU was recorded on board its own microSD card. The event data had to be combined in post processing. When the IMU system was powered up, small discrepancies in boot up time made the recordings start at different times. The data was realigned temporally by taking the accelerometer data into the frequency domain with an FFT and comparing the power spectral density such that the IMUs could be realigned based on the initial movement recorded. It can be seen in figure 3.2 at the two second time stamp the four IMUs in alignment with their respective initial peaks.

\subsubsection{Run 1}

Figure 3.3 shows each IMU and the data therein recorded. Each sub figure displays the raw data and thus are the rotational rates from their own respective frames of reference. Note the relative 


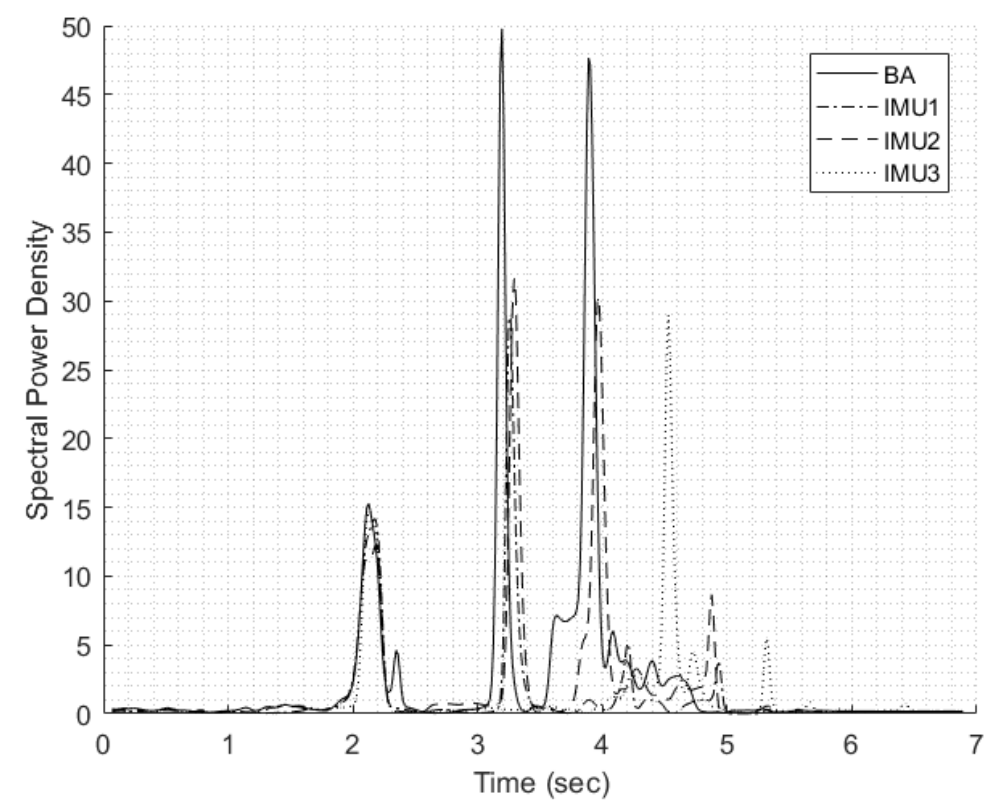

Figure 3.2: Run 1: Frequency domain analysis for temporal alignment of sensors

differences between each IMU as they experience the same event but from a different vantage point corresponding to their offset angles.

Observe run 1 operates below the gyroscope saturation limit for the duration of the experiment. There are no losses of data on any IMU channel as would be expected. There is a small dropout of data from Vicon at approximately 2.85 seconds before reestablishing visuals and hitting the arresting net at 3.05 seconds.

The IMU KF signal can be seen trending with the Vicon signal in Figures 3.5, 3.6, and 3.7. 

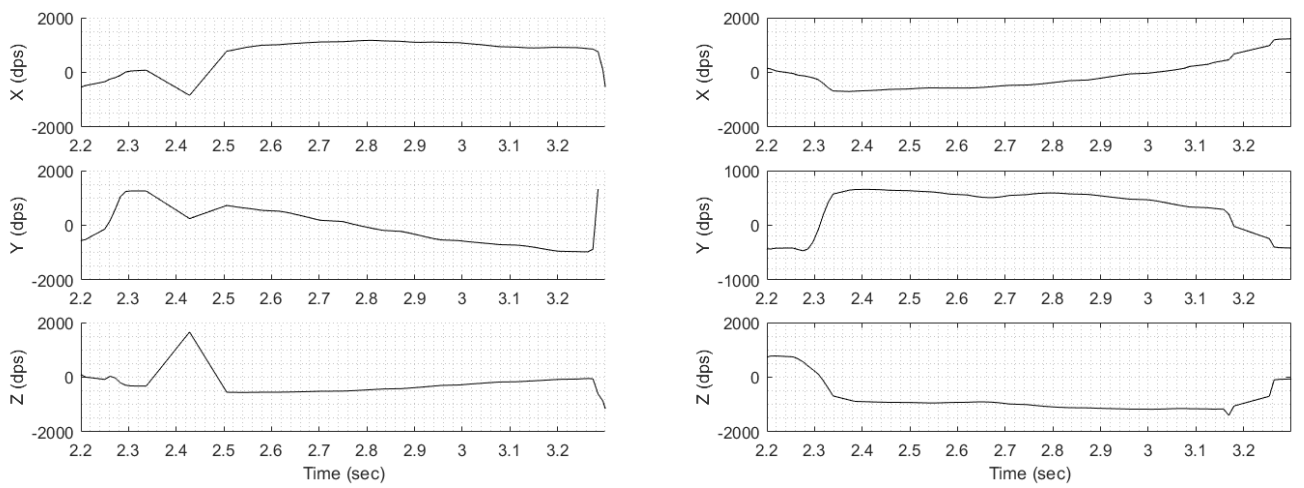

(a) IMU: body axis

(b) IMU: 1
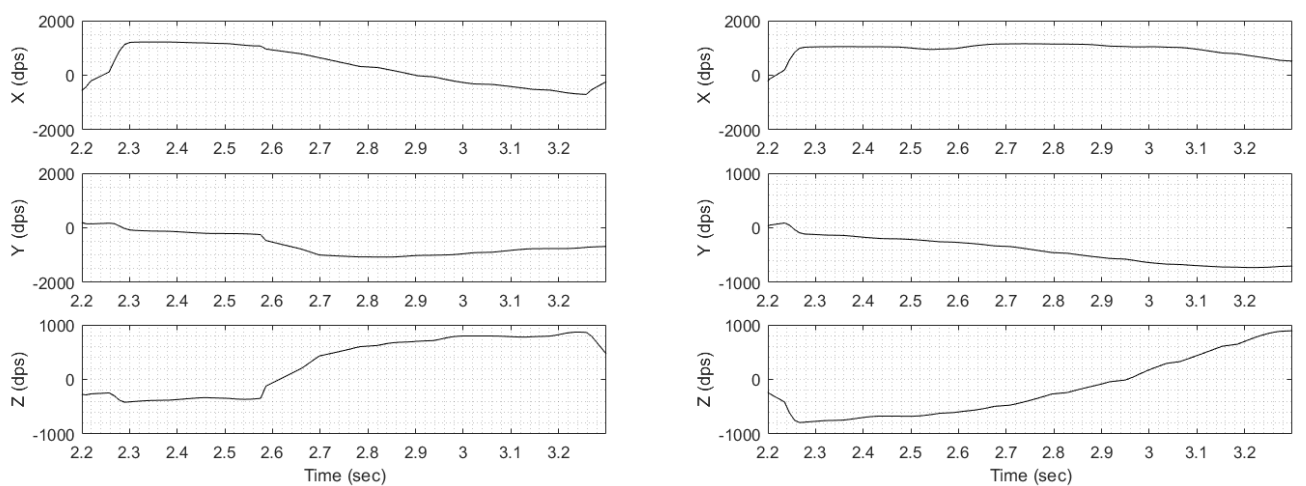

(c) IMU: 2

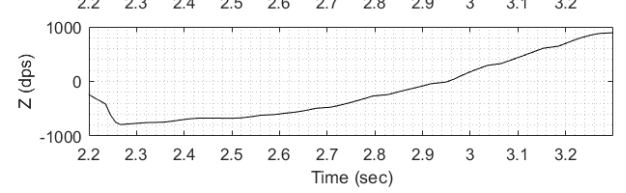

(d) IMU: 3

Figure 3.3: Run 1: Relative IMU ROI data
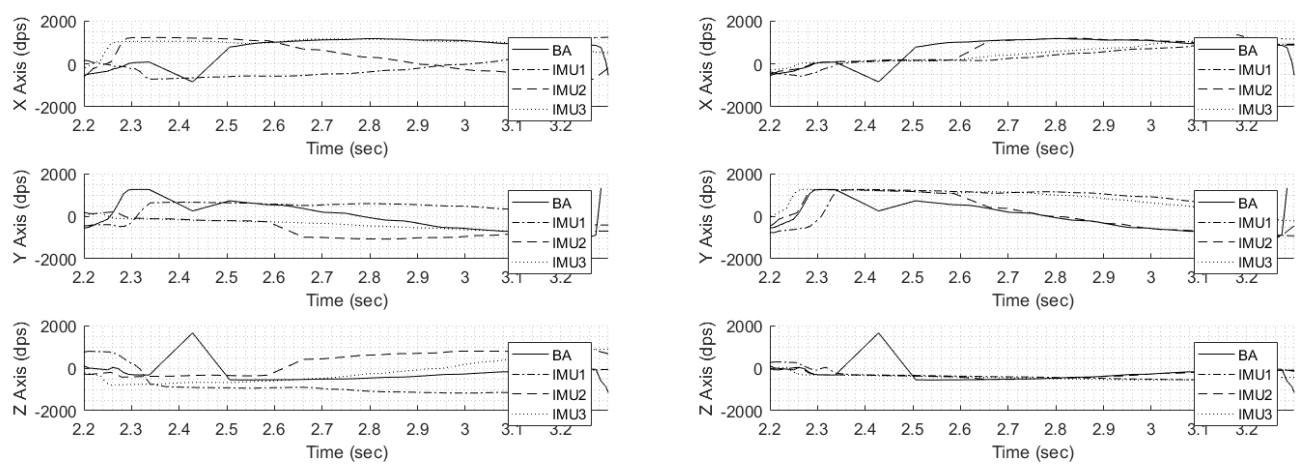

(a) Values recorded by individual sensors

(b) Values converted to common reference frame

Figure 3.4: Run 1: IMU data as recorded (a) and after conversion to common reference frame (b) 


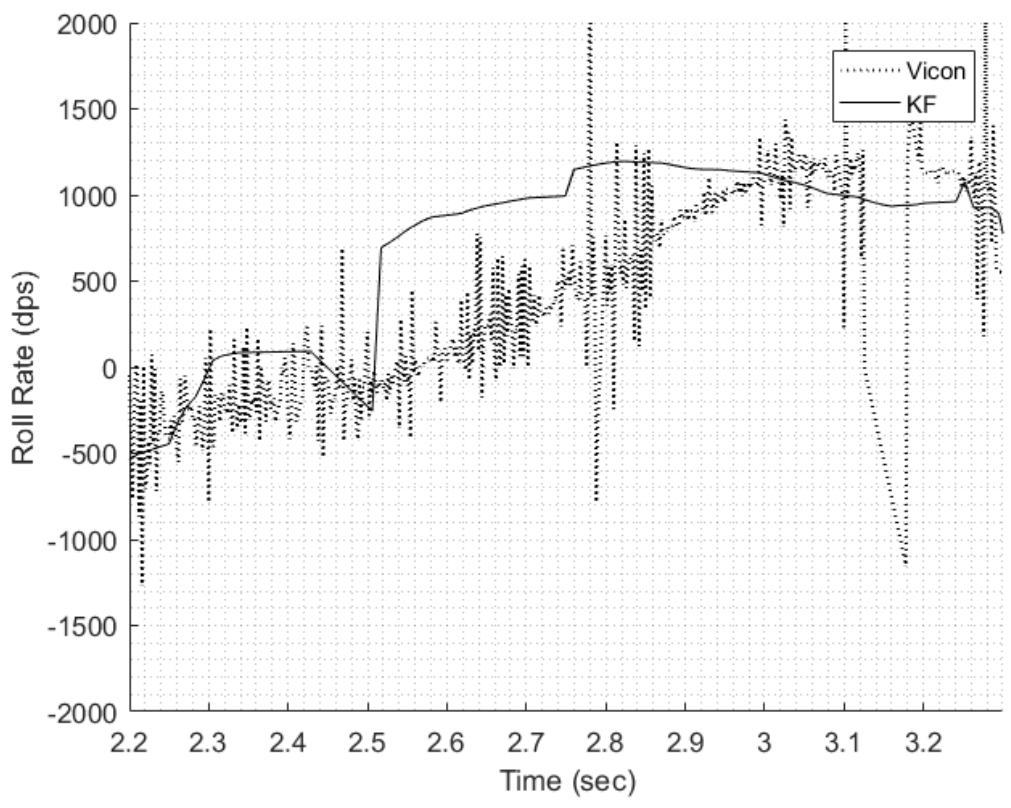

Figure 3.5: Run 1: Rotation about the $\mathrm{x}$ axis

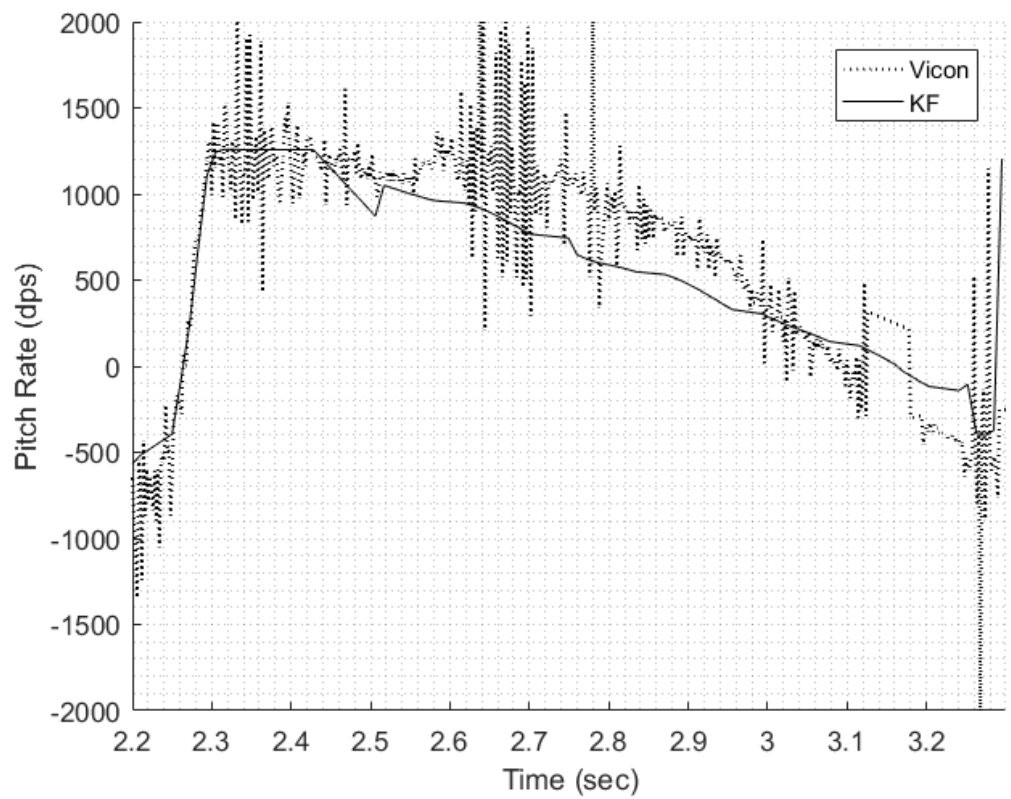

Figure 3.6: Run 1: Rotation about the y axis 


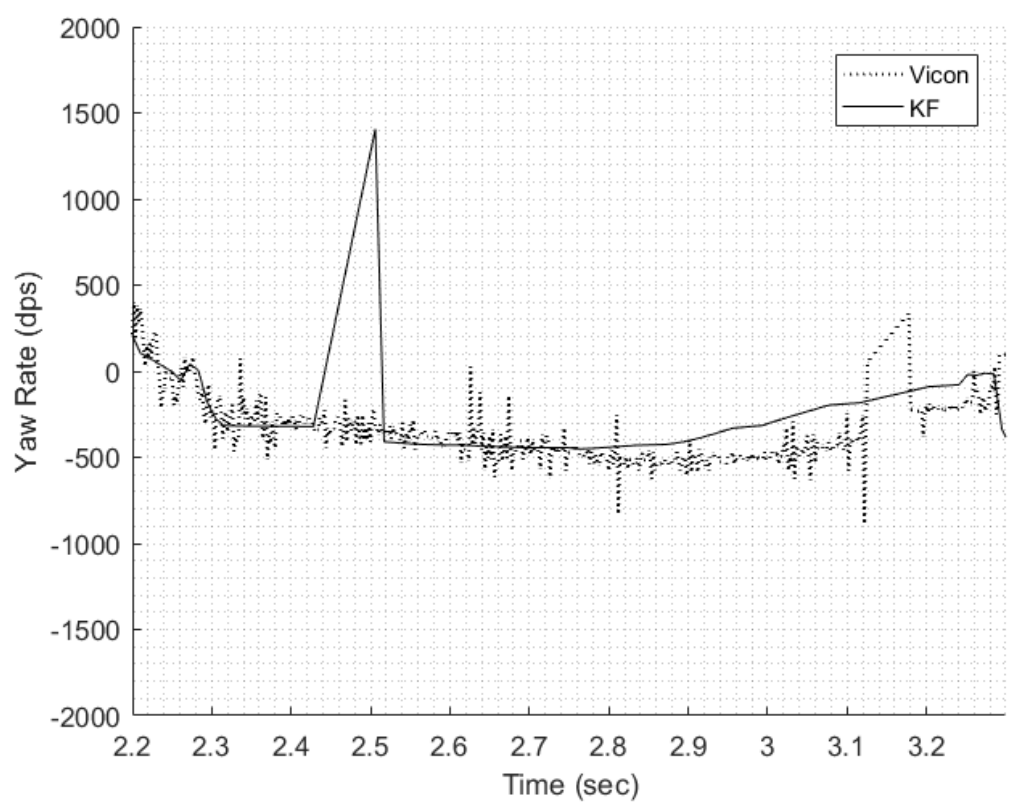

Figure 3.7: Run 1: Rotation about the $\mathrm{z}$ axis
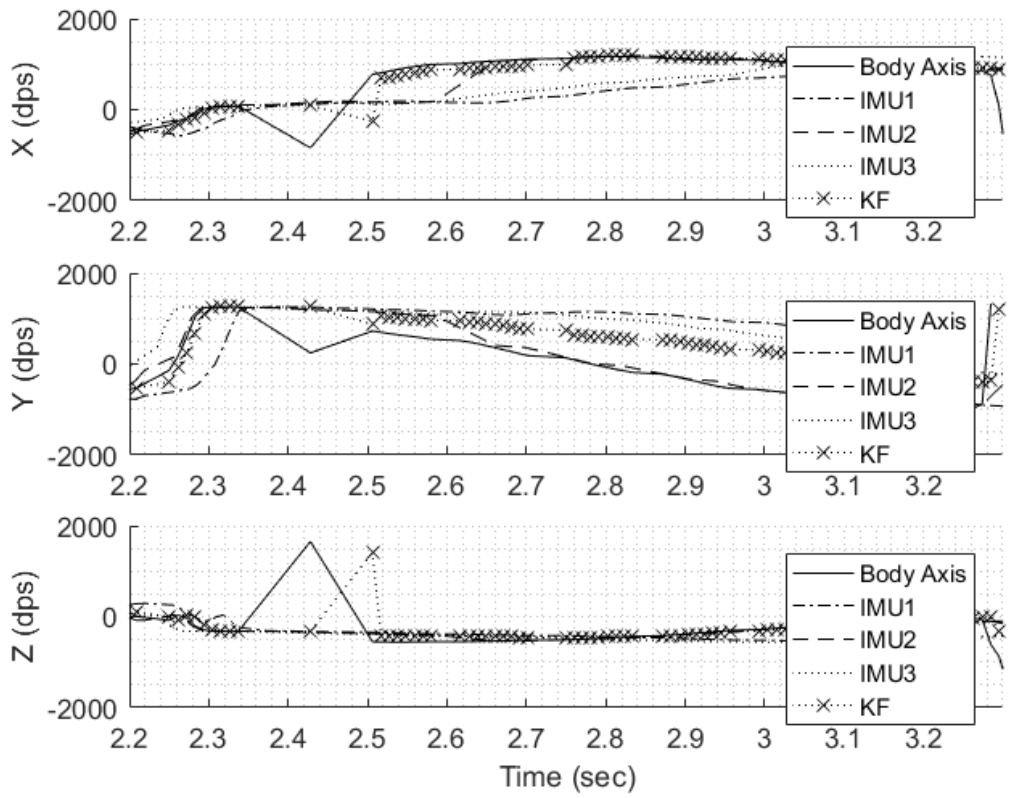

Figure 3.8: Run 1: Converted IMU data and Kalman Filter data fit 


\subsubsection{Run 2}

Note the common initiation point in figure 3.9 which was used to align the measurements temporally. Although the measurements start at the same time, it can be seen that there are varying degrees of input. This was noted as an indicator for potentially poor sensor fusion.

The body axis measurements during run 2 were complete on the roll and pitch axis but the yaw axis was saturated until the payload was stopped by the arresting net. The remaining IMU sensors either had one channel saturated or a combination of multiple channels switching between saturated and unsaturated. Figure 3.11 (b) shows the converted IMU 1 and IMU 2 having a small portion of success with yaw recordings fluctuating around $2500 \mathrm{dps}$, approximately $500 \mathrm{dps}$ above the saturation limit of the sensor.

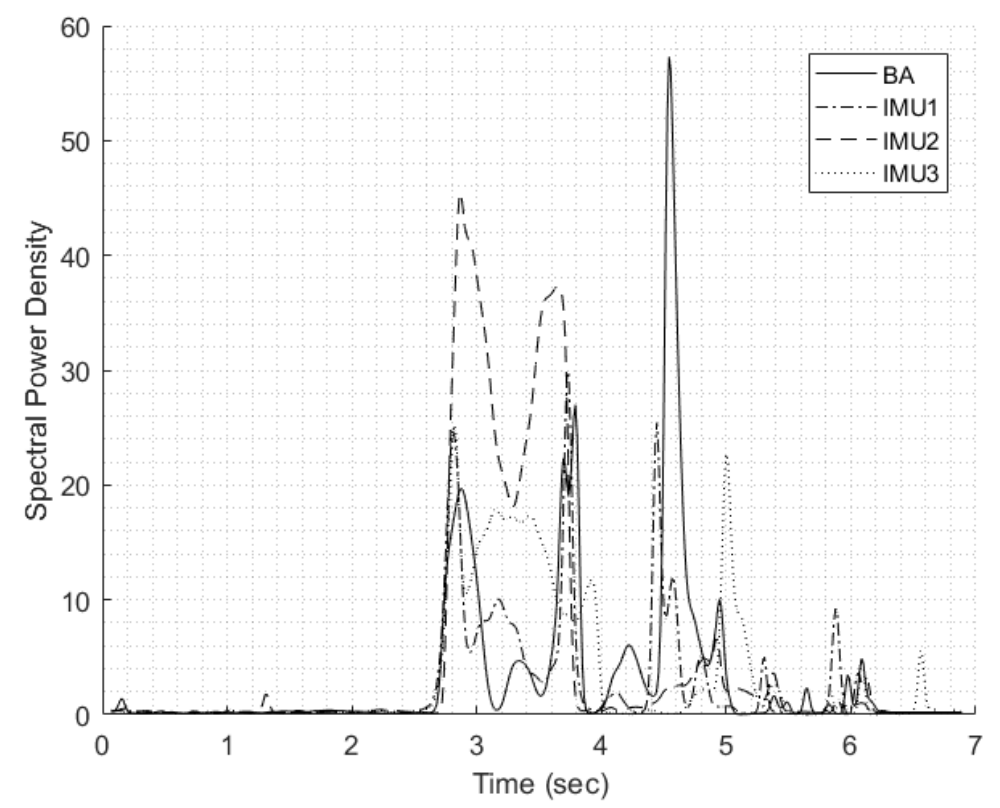

Figure 3.9: Run 2: Frequency domain analysis for temporal alignment of sensors 


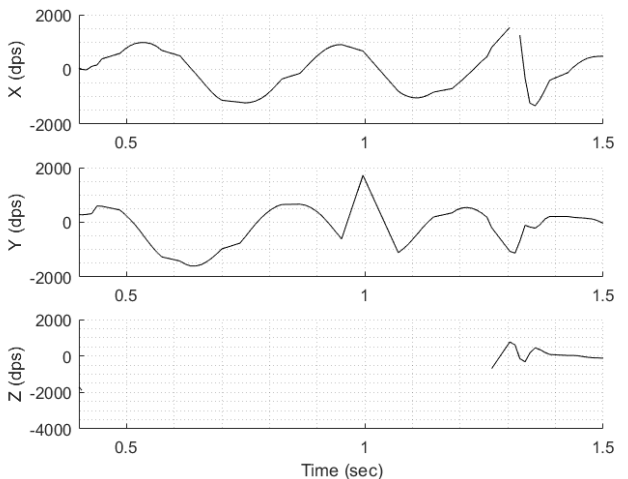

(a) IMU: body axis
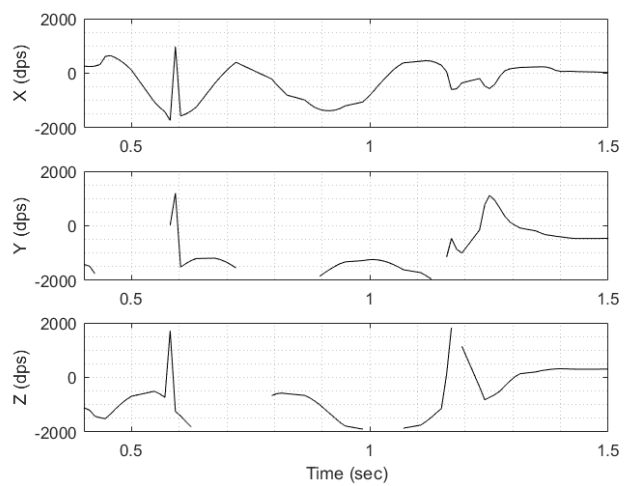

(c) IMU: 2
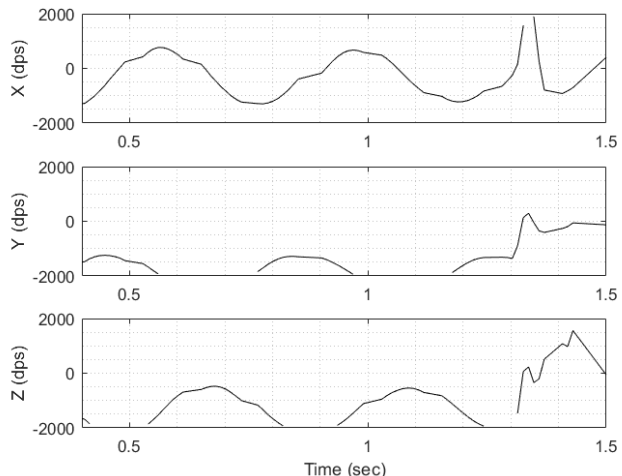

(b) IMU: 1
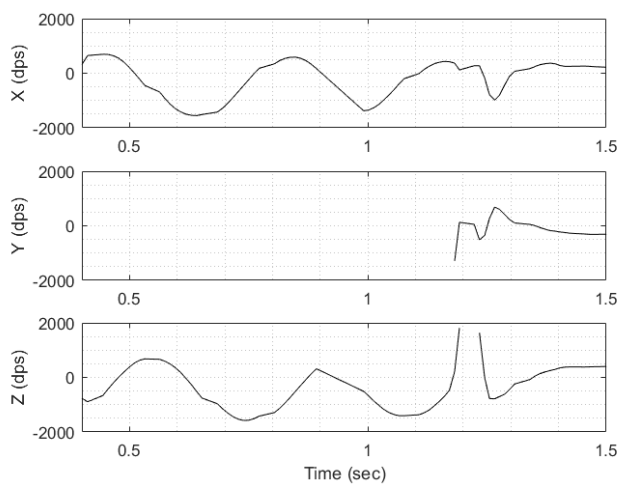

(d) IMU: 3

Figure 3.10: Run 2: Relative IMU ROI data
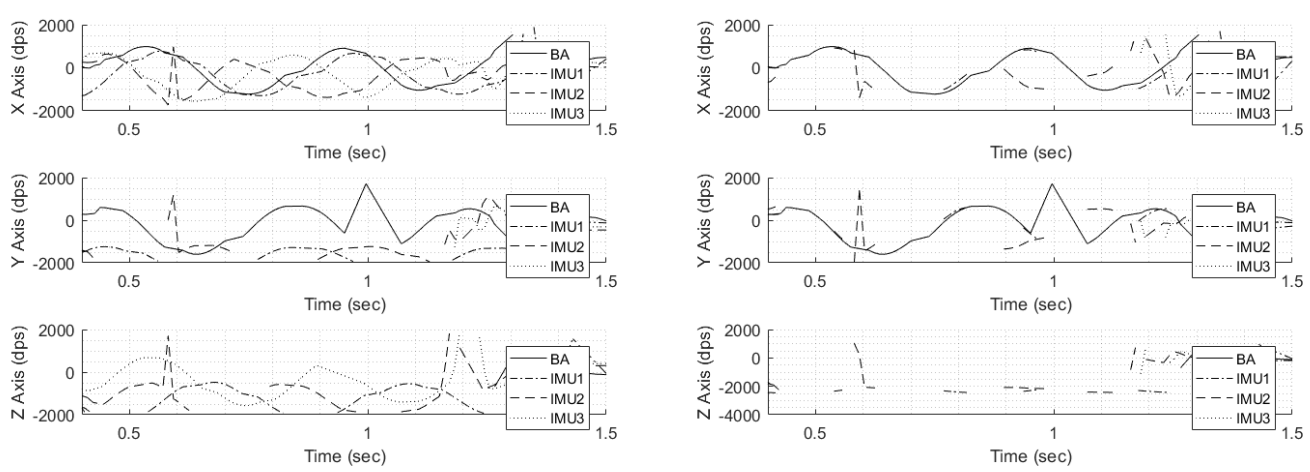

(a) Values recorded by individual sensors

(b) Values converted to common reference frame

Figure 3.11: Run 2: IMU data as recorded (a) and after conversion to common reference frame (b) 


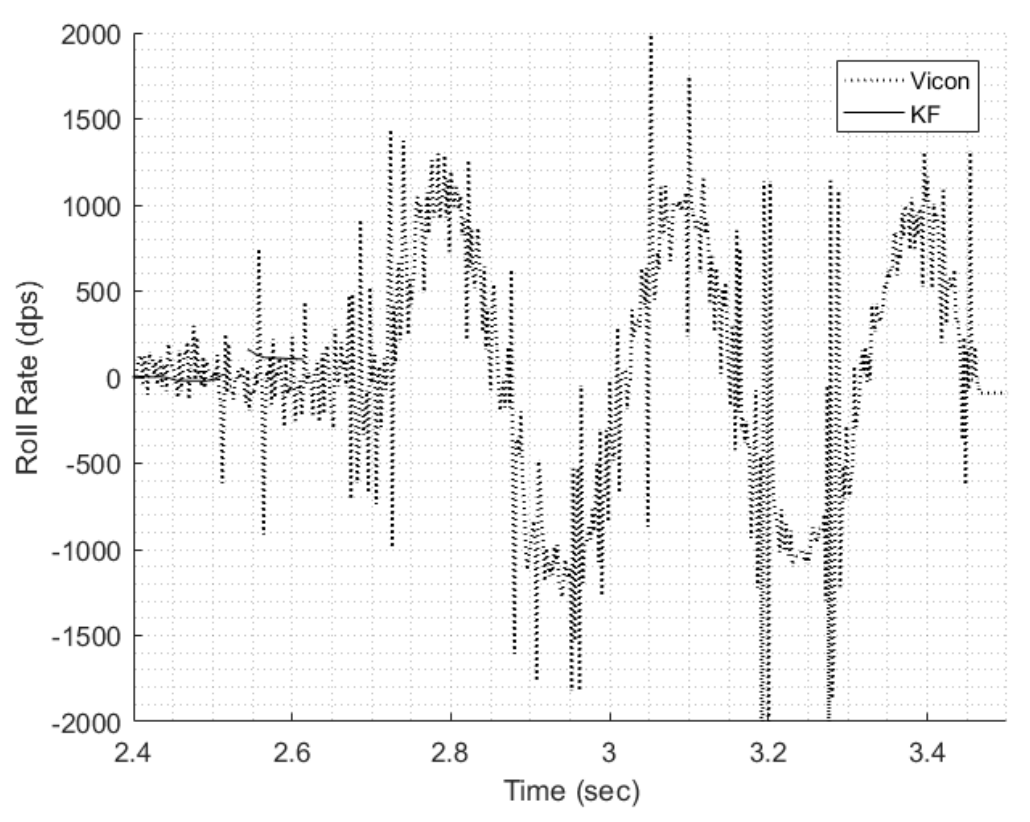

Figure 3.12: Run 2: Rotation about the $\mathrm{x}$ axis

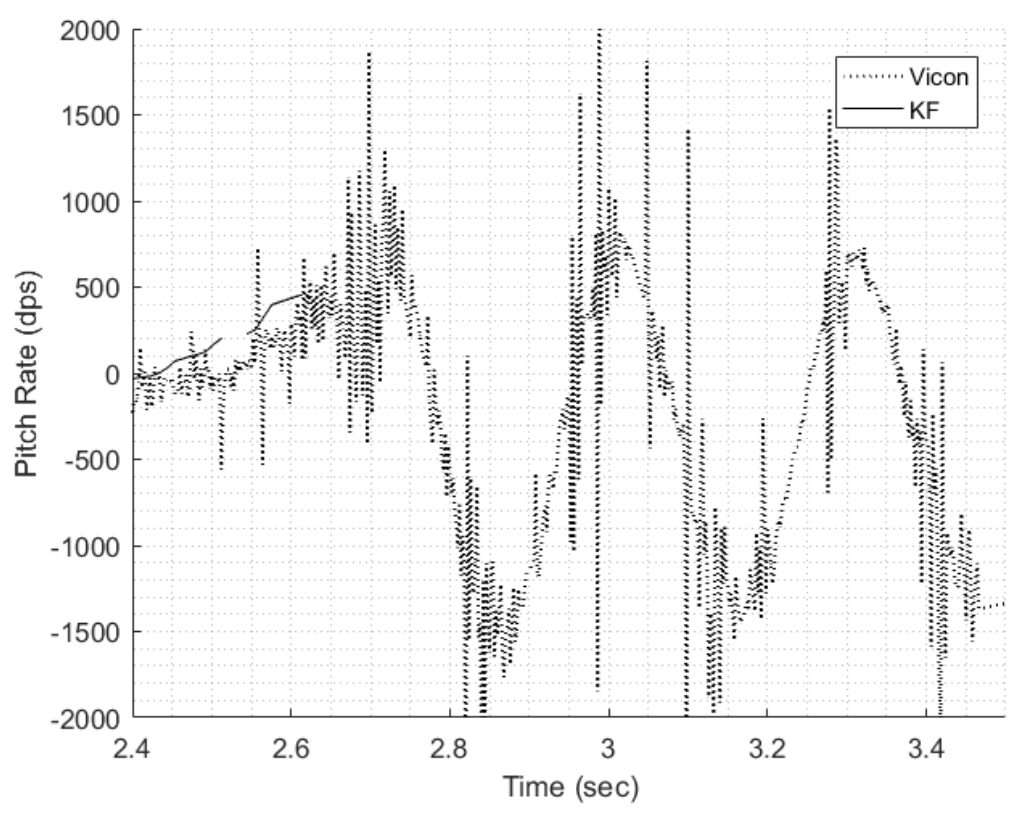

Figure 3.13: Run 2: Rotation about the y axis 


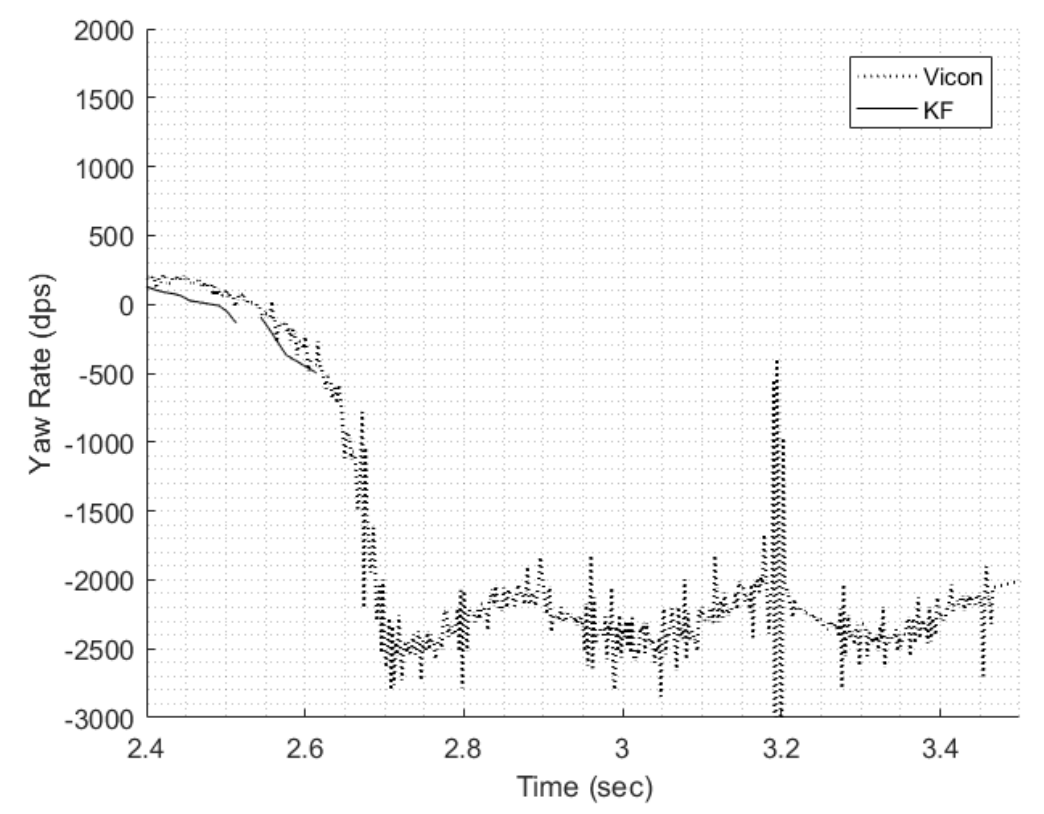

Figure 3.14: Run 2: Rotation about the $\mathrm{z}$ axis
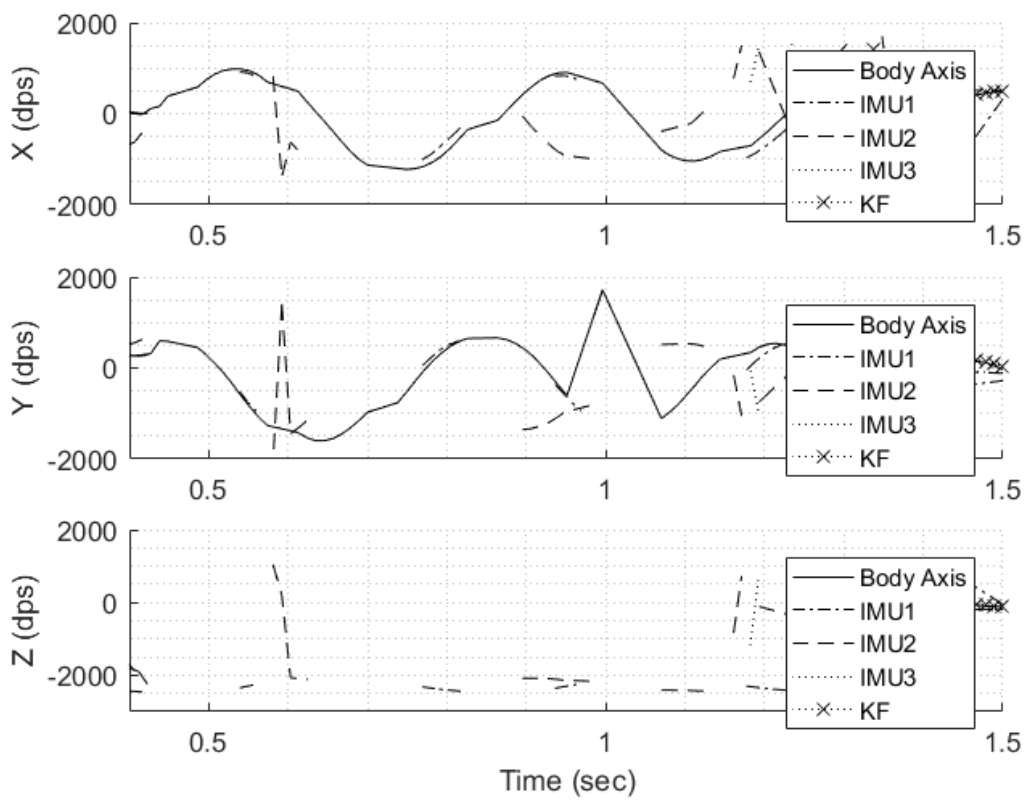

Figure 3.15: Run 2: Converted IMU data and Kalman Filter data fit 


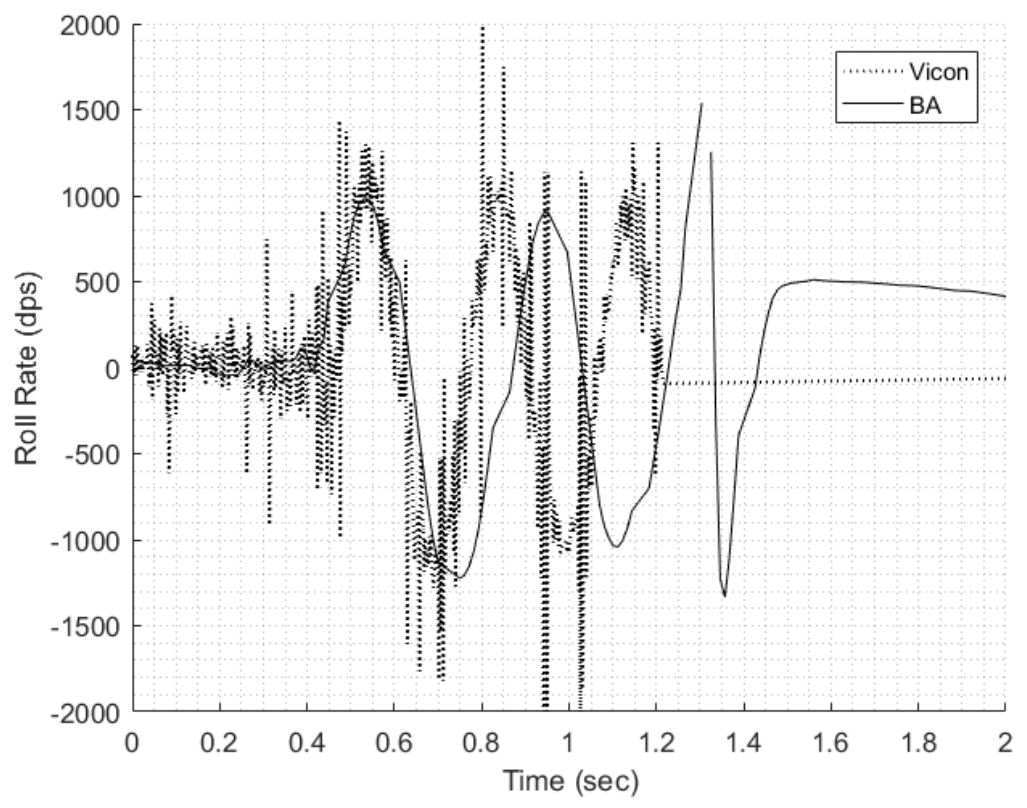

Figure 3.16: Run 2: Vicon and body axis roll 


\subsubsection{Run 3}

Run 3 as seen in figure 3.18 saturates on more than one channel. Figures 3.20, 3.21, and 3.22 also show the roll axis and yaw axis being saturated for some or all of the test with data exceeding 3000 dps in some instances.

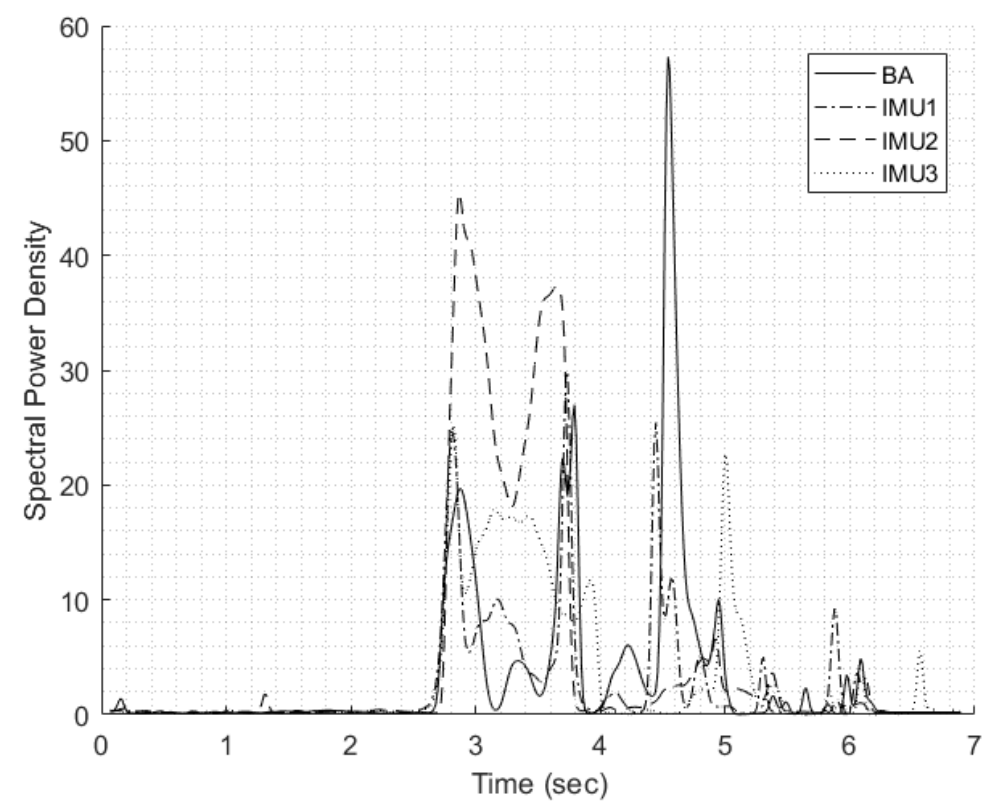

Figure 3.17: Run 3: Frequency domain analysis for temporal alignment of sensors 

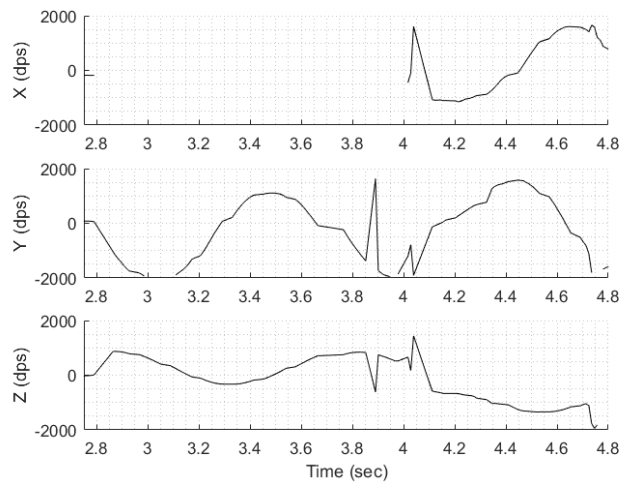

(a) IMU: body axis
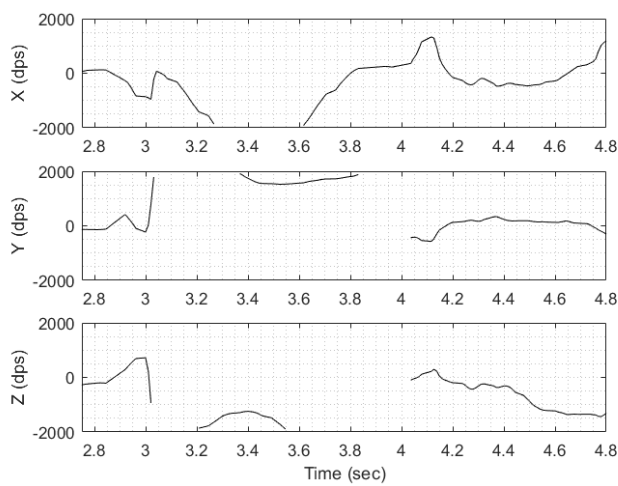

(c) IMU: 2
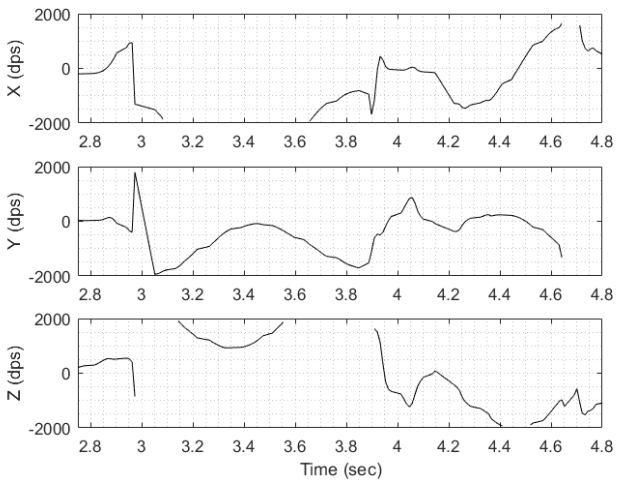

(b) IMU: 1
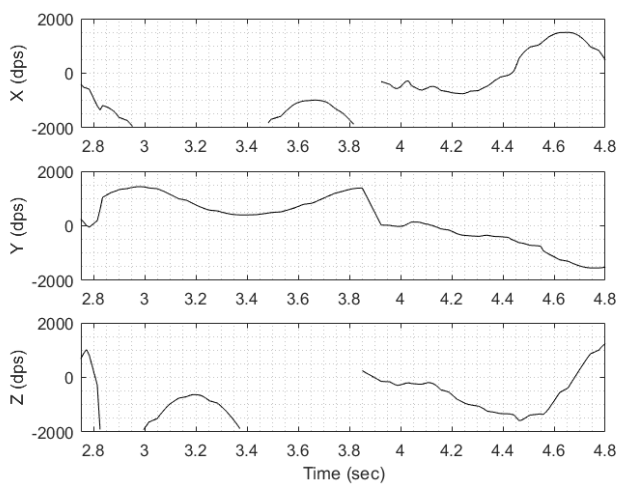

(d) IMU: 3

Figure 3.18: Run 3: Relative IMU ROI data
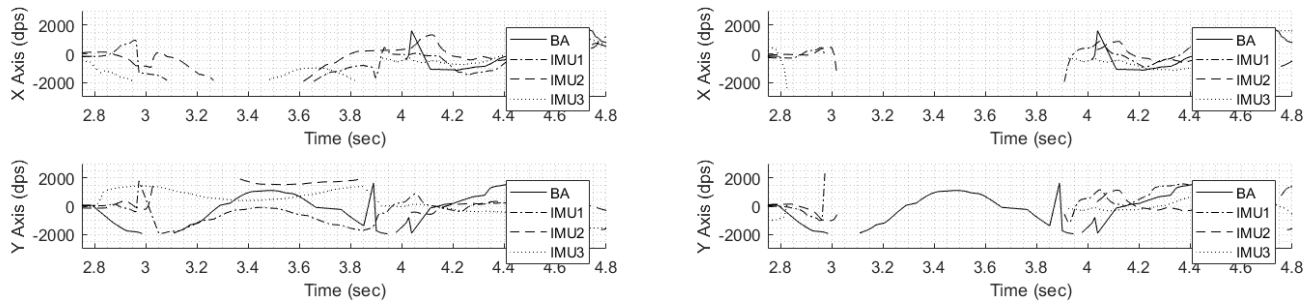

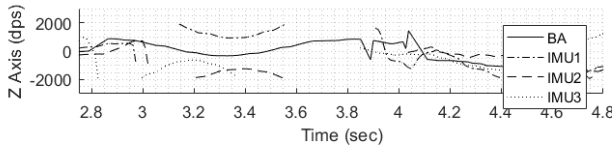

(a) Values recorded by individual sensors

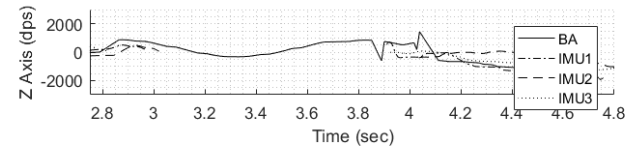

(b) Values converted to common reference frame

Figure 3.19: Run 3: IMU data as recorded (a) and after conversion to common reference frame (b) 


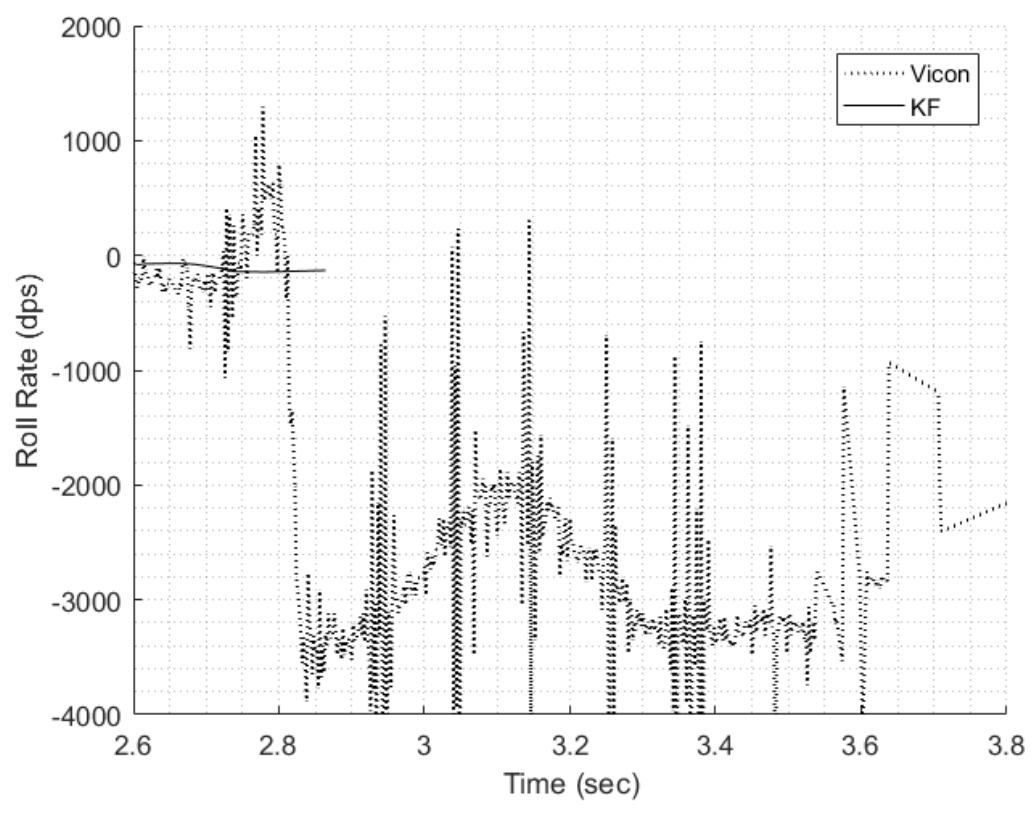

Figure 3.20: Run 3: Rotation about the $\mathrm{x}$ axis

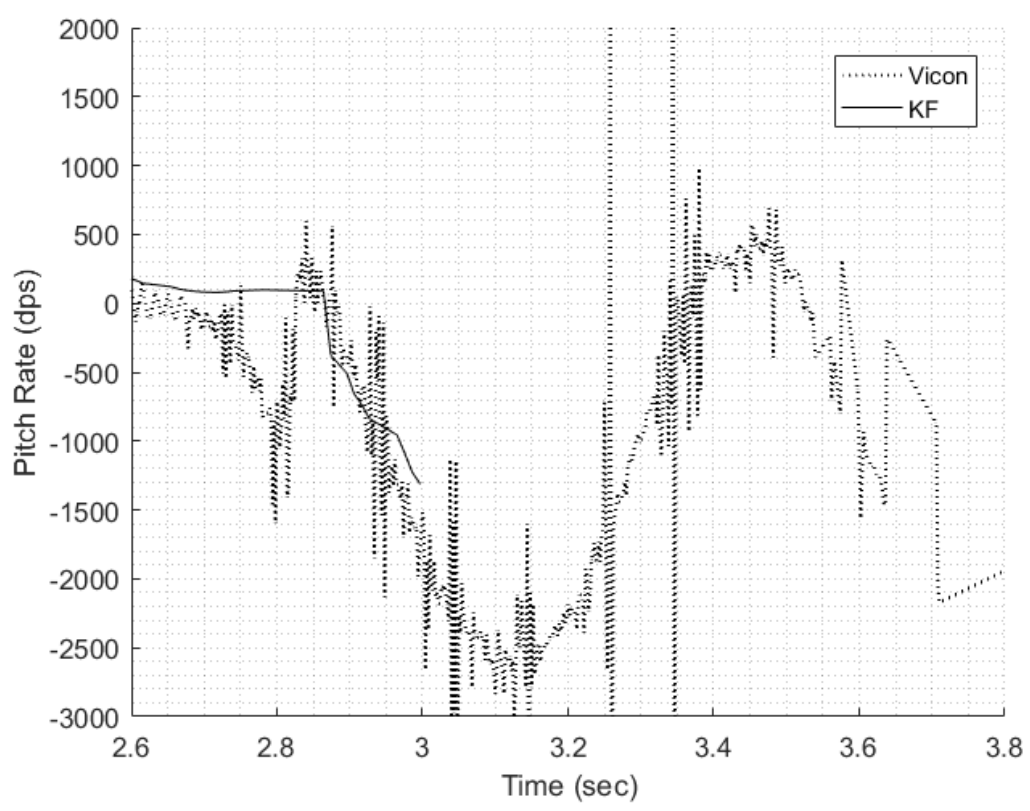

Figure 3.21: Run 3: Rotation about the y axis 


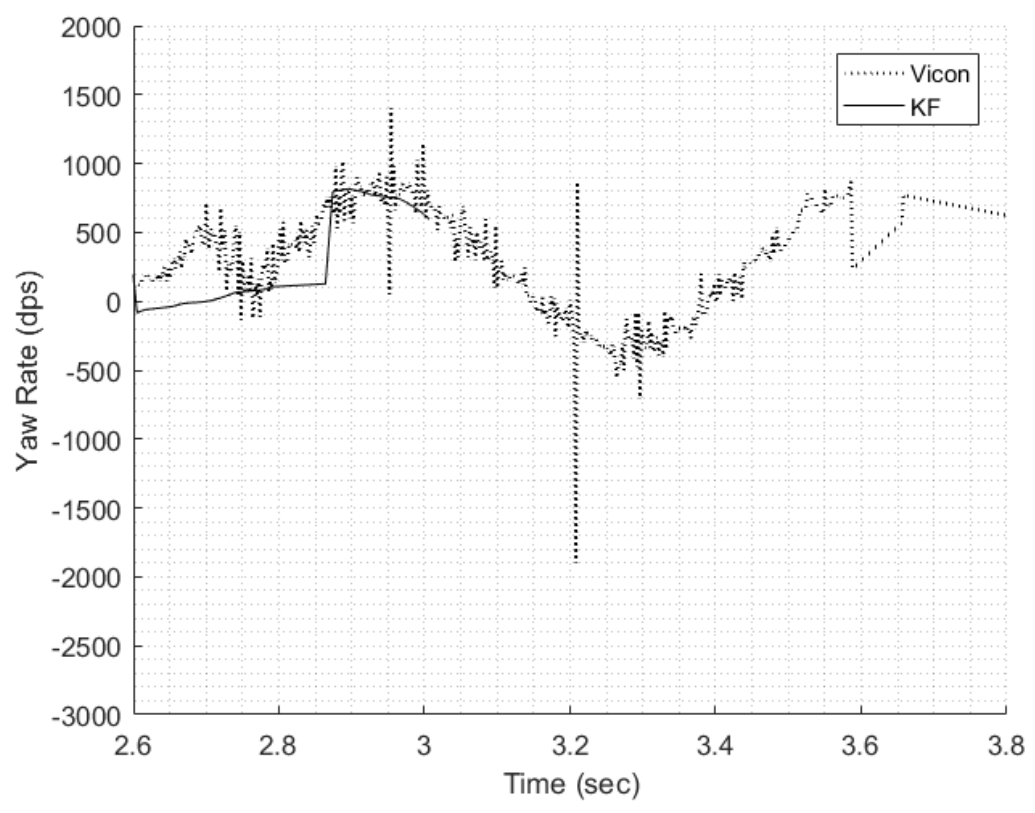

Figure 3.22: Run 3: Rotation about the $\mathrm{z}$ axis
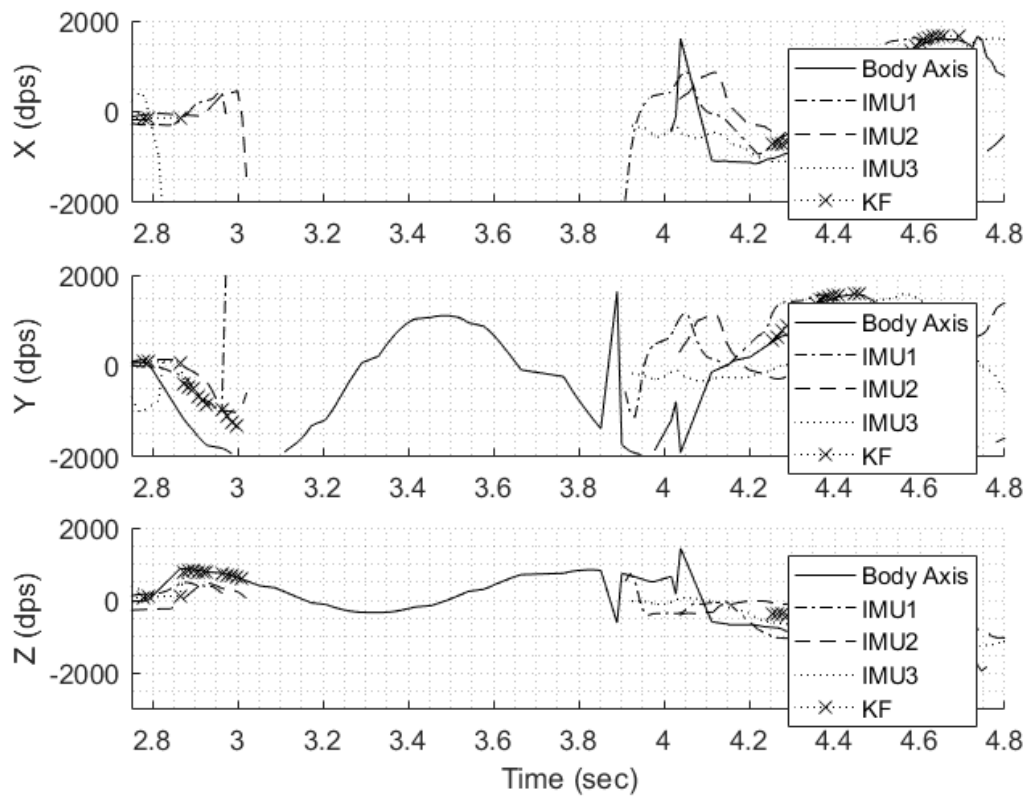

Figure 3.23: Run 3: Converted IMU data and Kalman Filter data fit 


\subsubsection{Run 4}

Run 4 has two channels on the body axis and IMU 3 that are relatively benign with one channel being saturated. Run 4 is similar to run 2 in that the channel that is saturated is experiencing rotational rates around $2500 \mathrm{dps}$ with moderate success in the converted rates reading around 2500 dps in figure 3.26 (b). The Kalman filter however does not perform well and does not present results during the test which can be seen in Figures 3.27, 3.28, and 3.29).

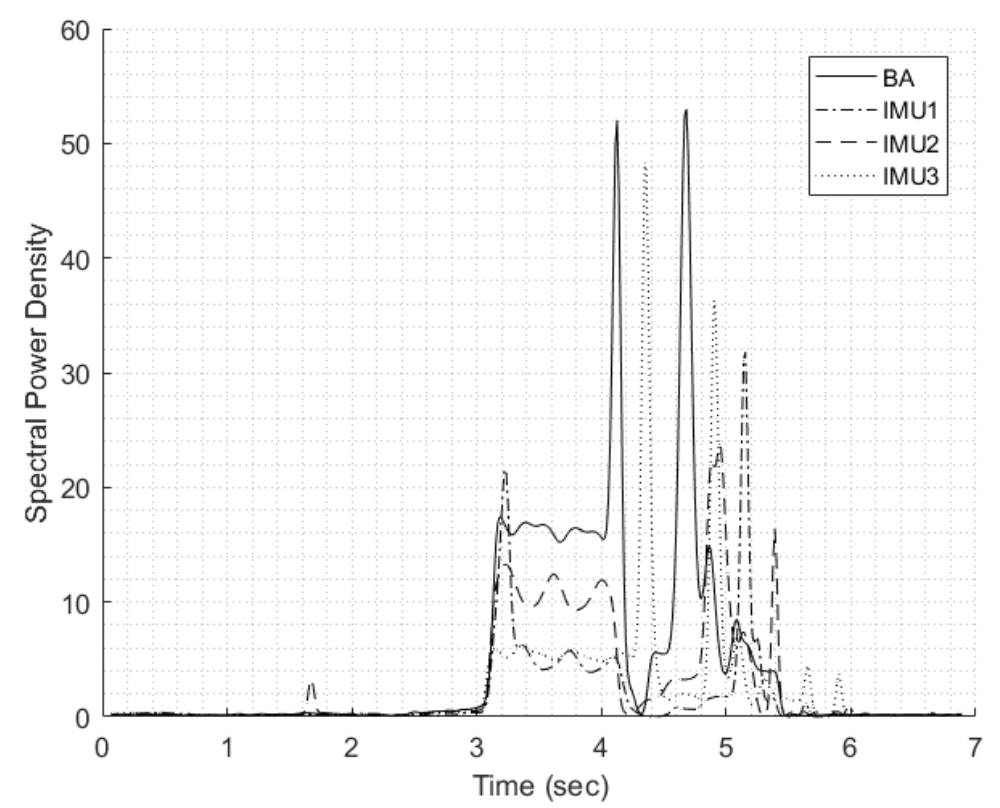

Figure 3.24: Run 4: Frequency domain analysis for temporal alignment of sensors 

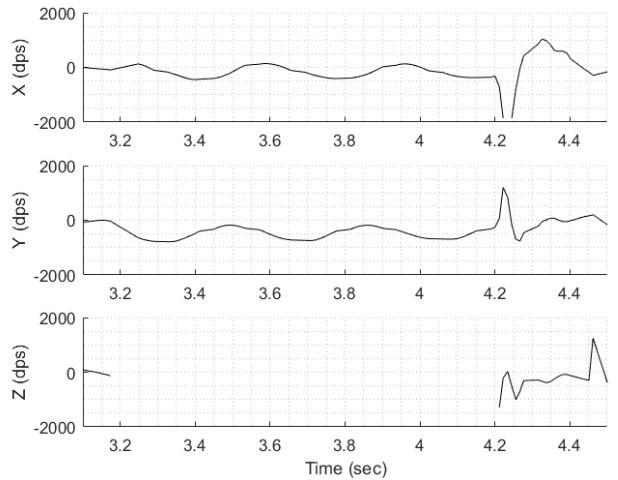

(a) IMU: body axis
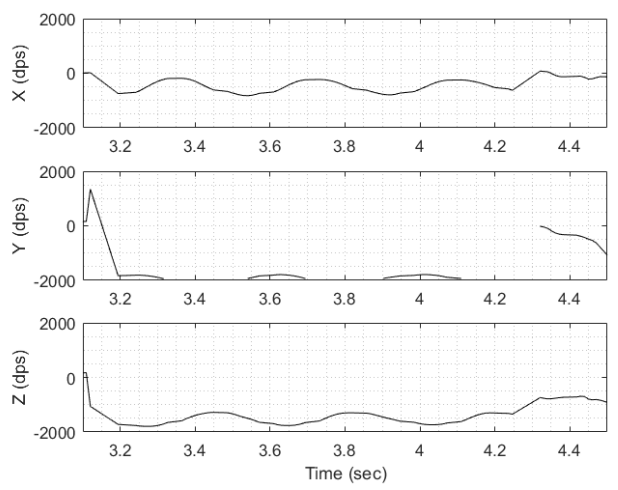

(c) IMU: 2
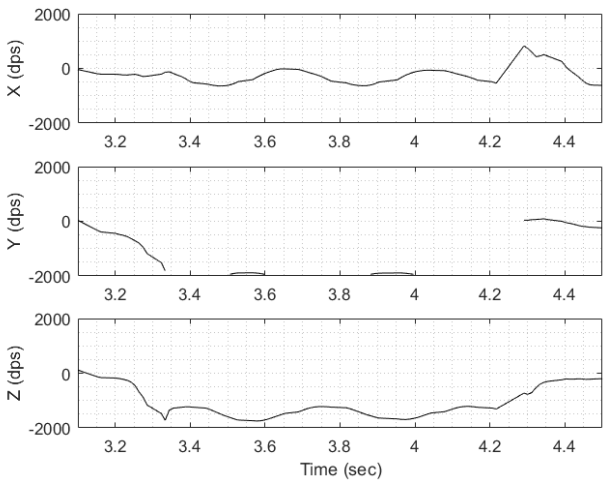

(b) IMU: 1
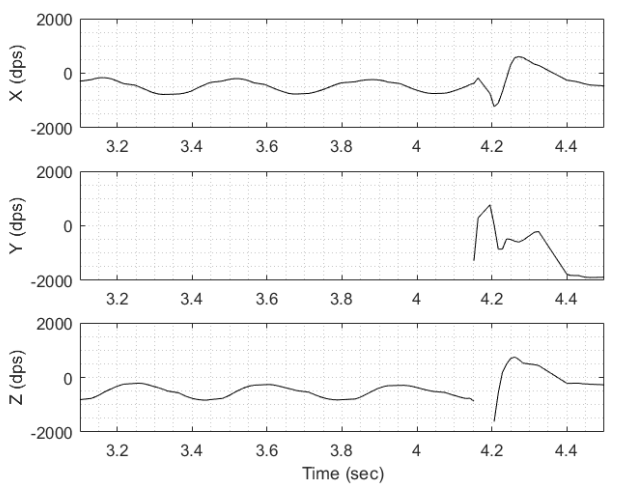

(d) IMU: 3

Figure 3.25: Run 3: ROI data
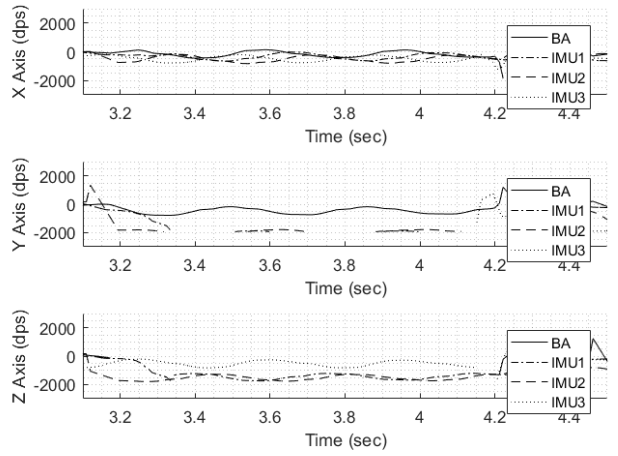

(a) Values recorded by individual sensors
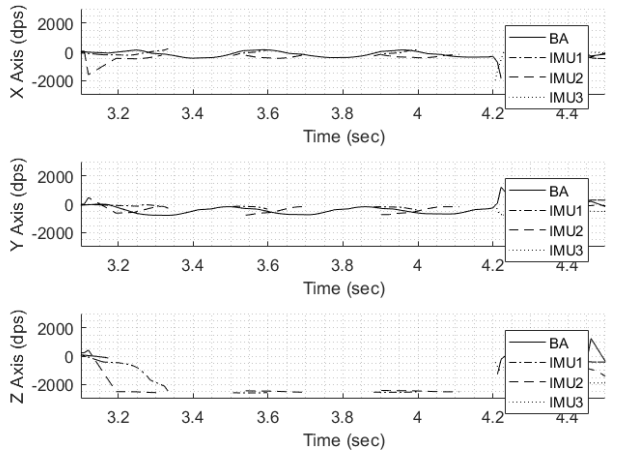

(b) Values converted to common reference frame

Figure 3.26: Run 4: IMU data as recorded (a) and after conversion to common reference frame (b) 


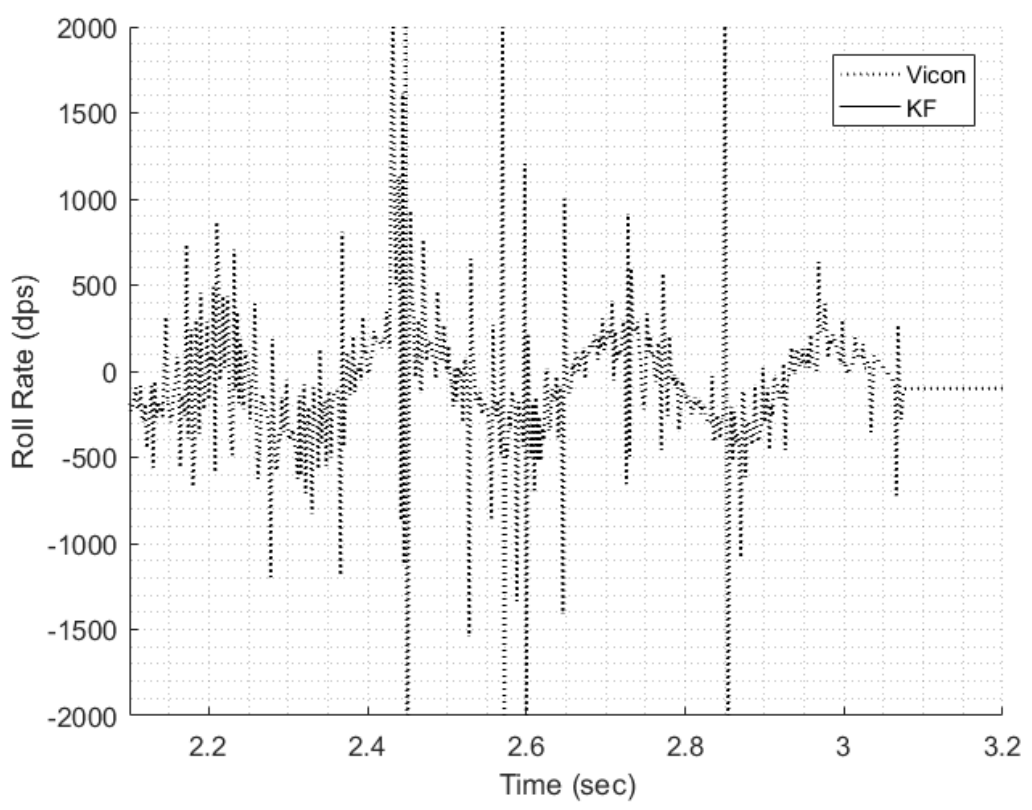

Figure 3.27: Run 4: Rotation about the $\mathrm{x}$ axis

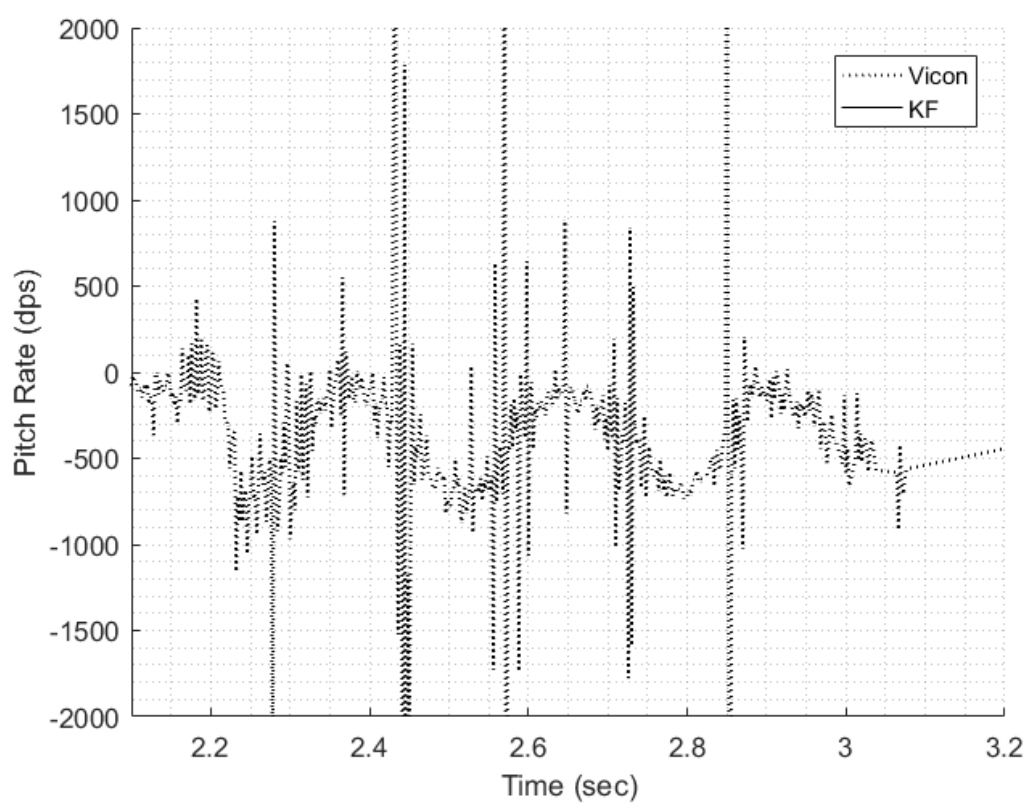

Figure 3.28: Run 4: Rotation about the y axis 


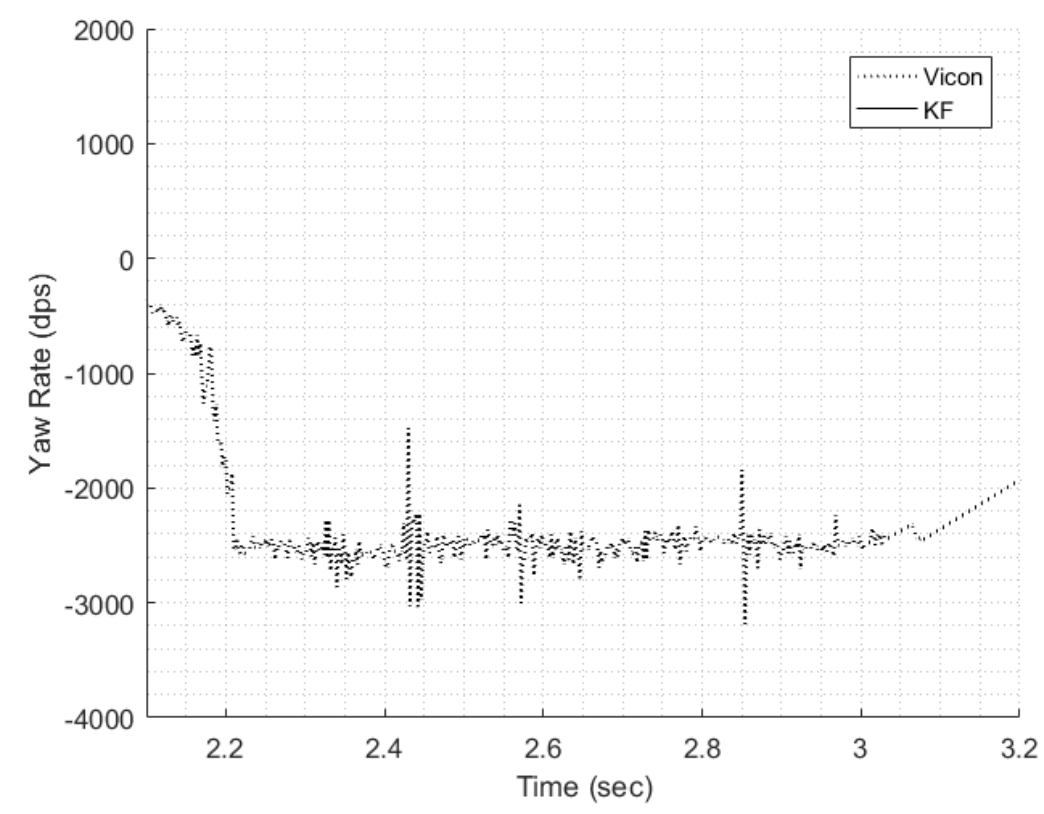

Figure 3.29: Run 4: Rotation about the $\mathrm{z}$ axis
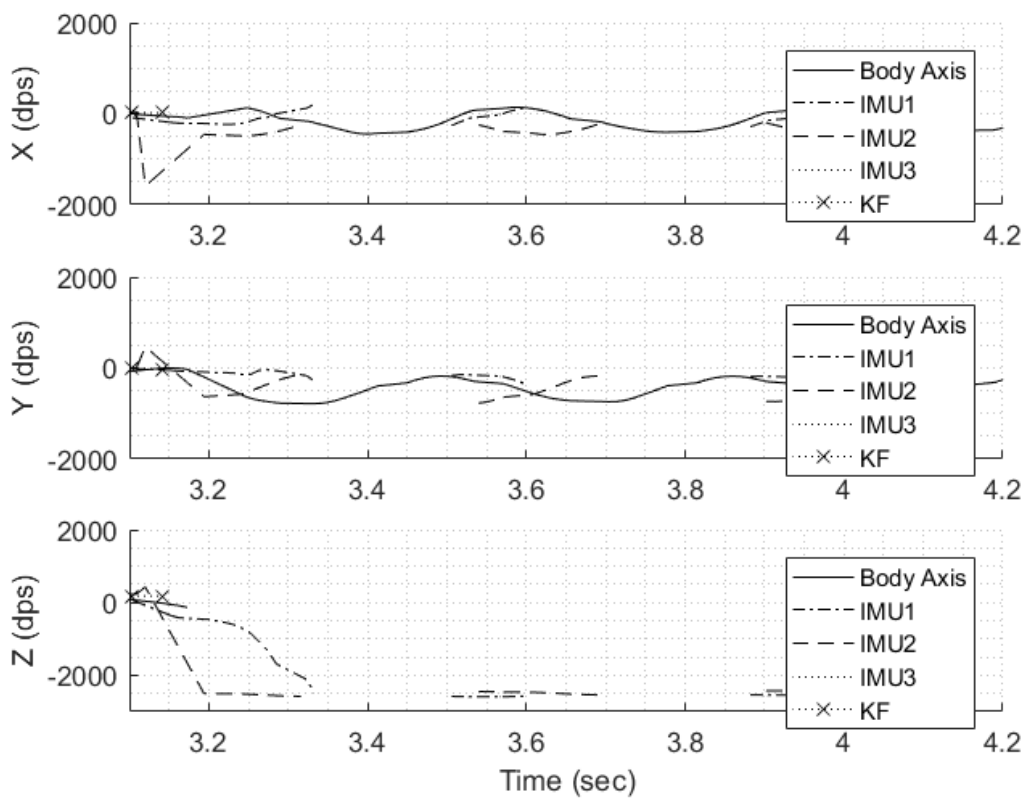

Figure 3.30: Run 4: Converted IMU data and Kalman Filter data fit 


\subsubsection{Run 5}

Run 5 has two channels saturated for various intervals for each IMU on the payload. The body axis IMU captured the trend of the roll rate which can be further seen in figure 3.38. The converted values shown in figure 3.33 have poor agreement with each other. The previous results can be seen exacerbated in figures $3.34,3.35$, and 3.36 showing a poor filtered fit to the Vicon data.

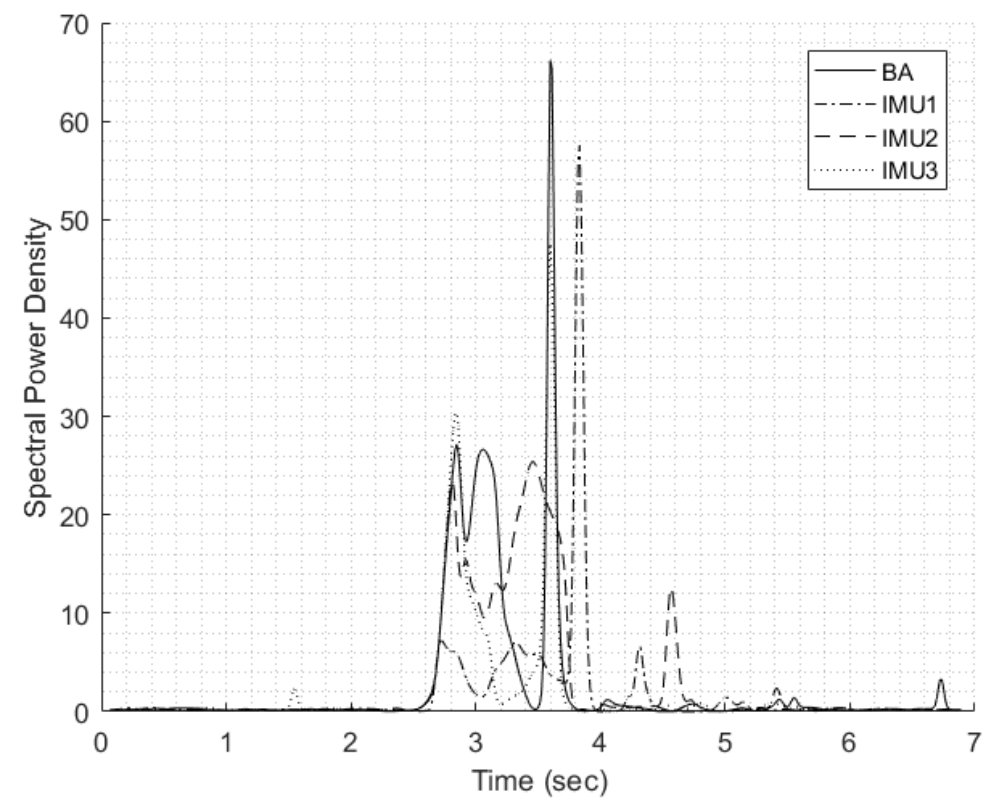

Figure 3.31: Run 5: Frequency domain analysis for temporal alignment of sensors 


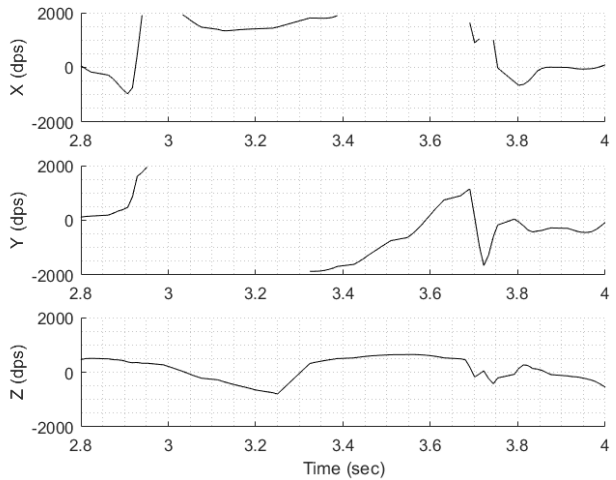

(a) IMU: body axis
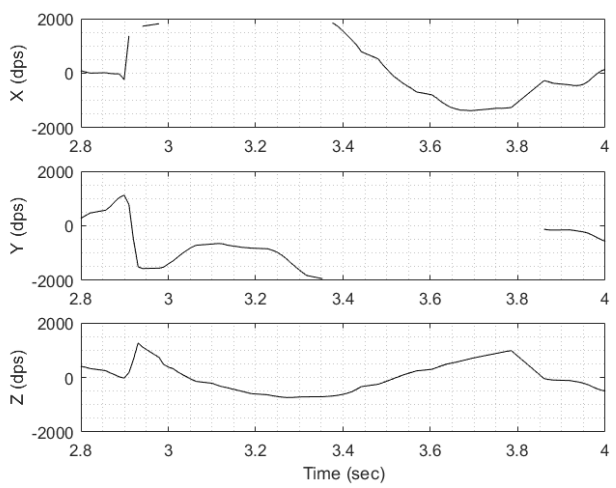

(c) IMU: 2
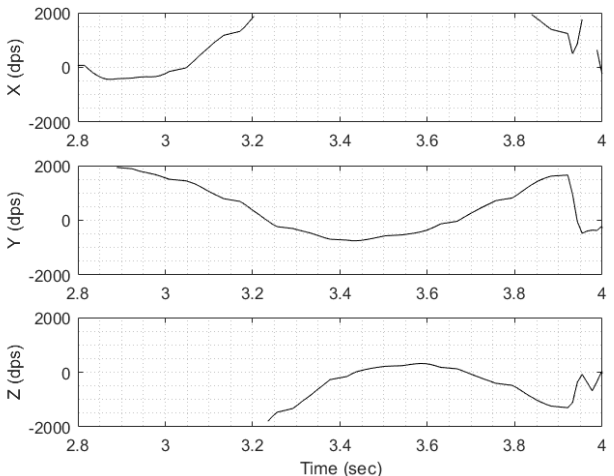

(b) IMU: 1
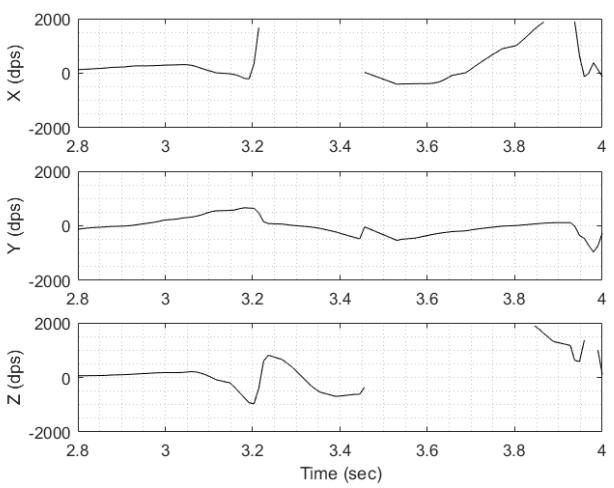

(d) IMU: 3

Figure 3.32: Run 5: Relative IMU ROI data
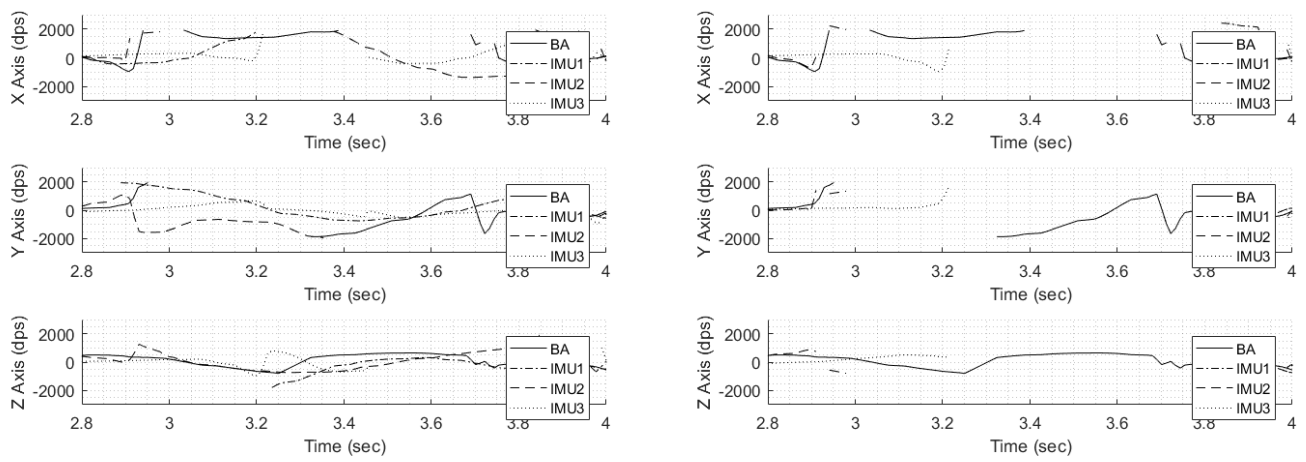

(a) Values recorded by individual sensors

(b) Values converted to common reference frame

Figure 3.33: Run 5: IMU data as recorded (a) and after conversion to common reference frame (b) 


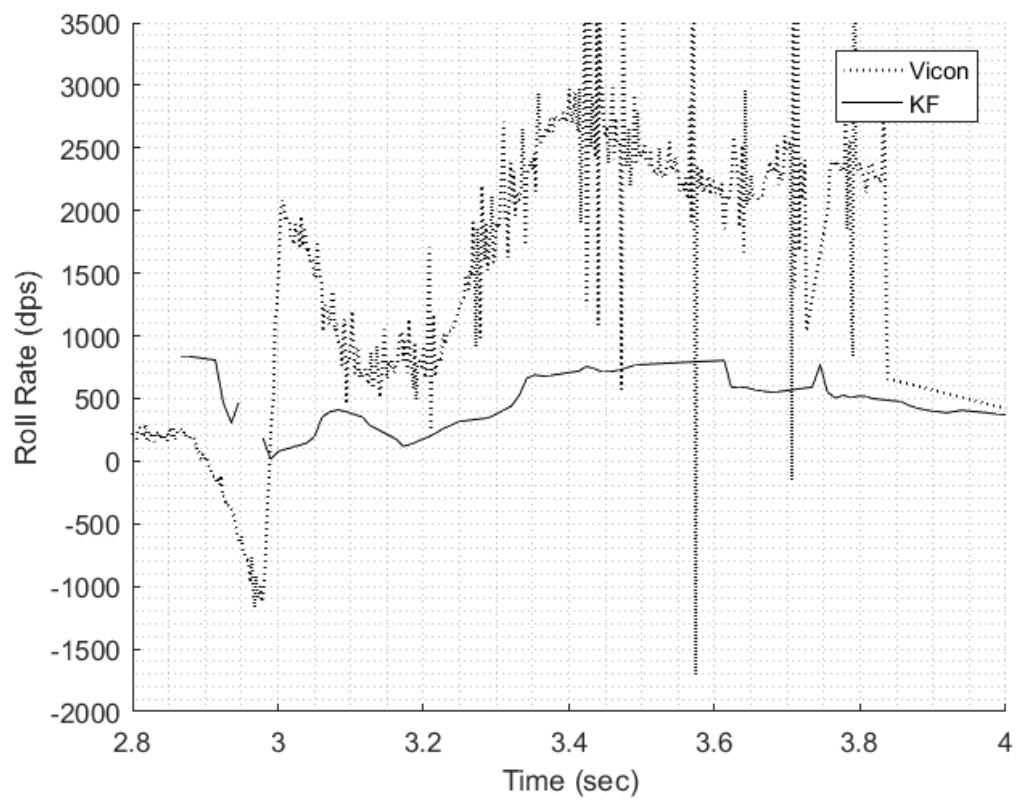

Figure 3.34: Run 5: Rotation about the $\mathrm{x}$ axis

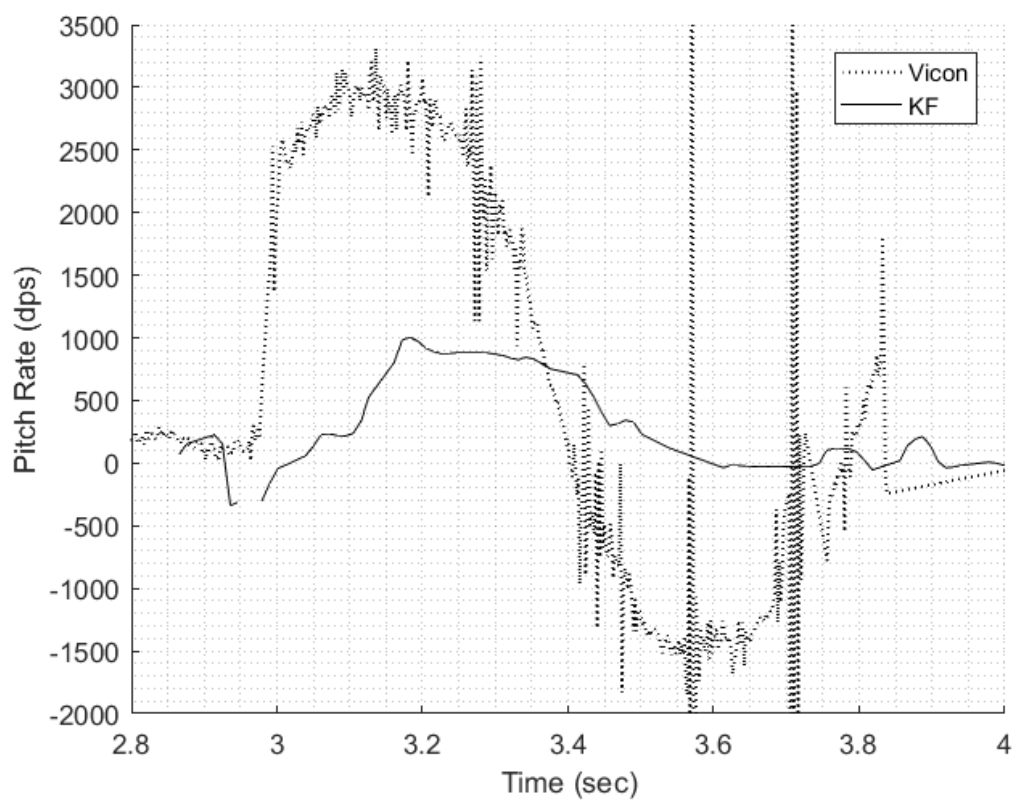

Figure 3.35: Run 5: Rotation about the y axis 


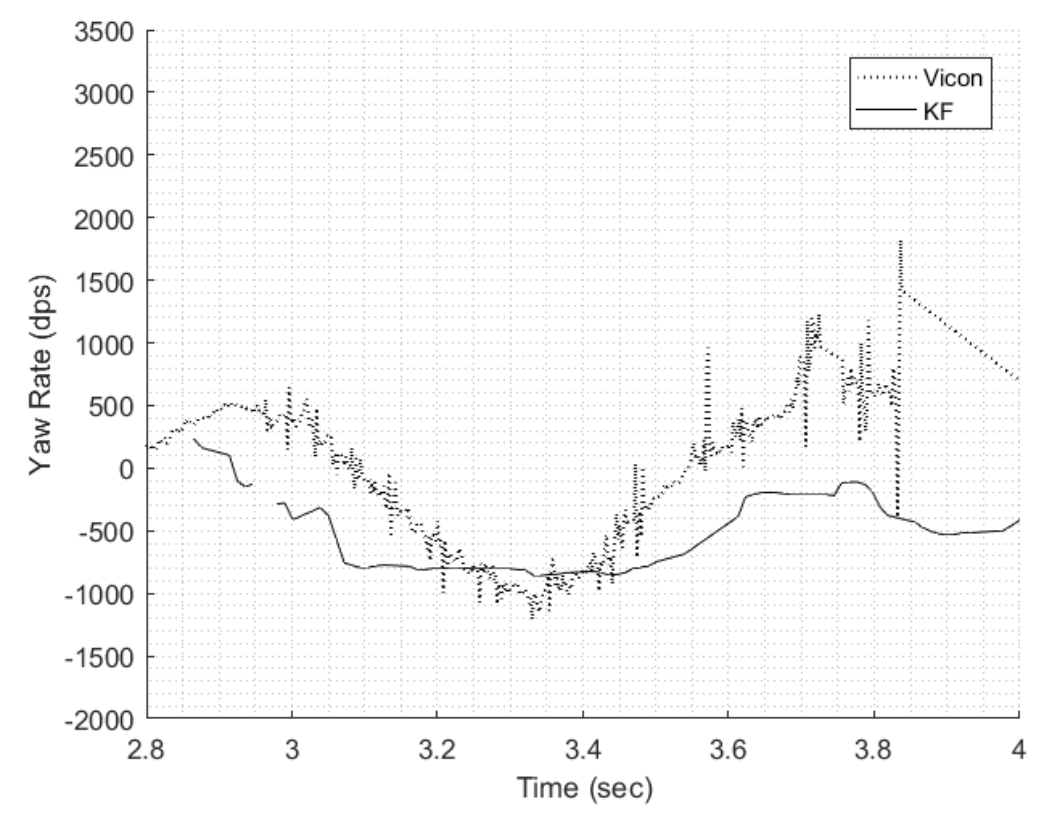

Figure 3.36: Run 5: Rotation about the $\mathrm{z}$ axis
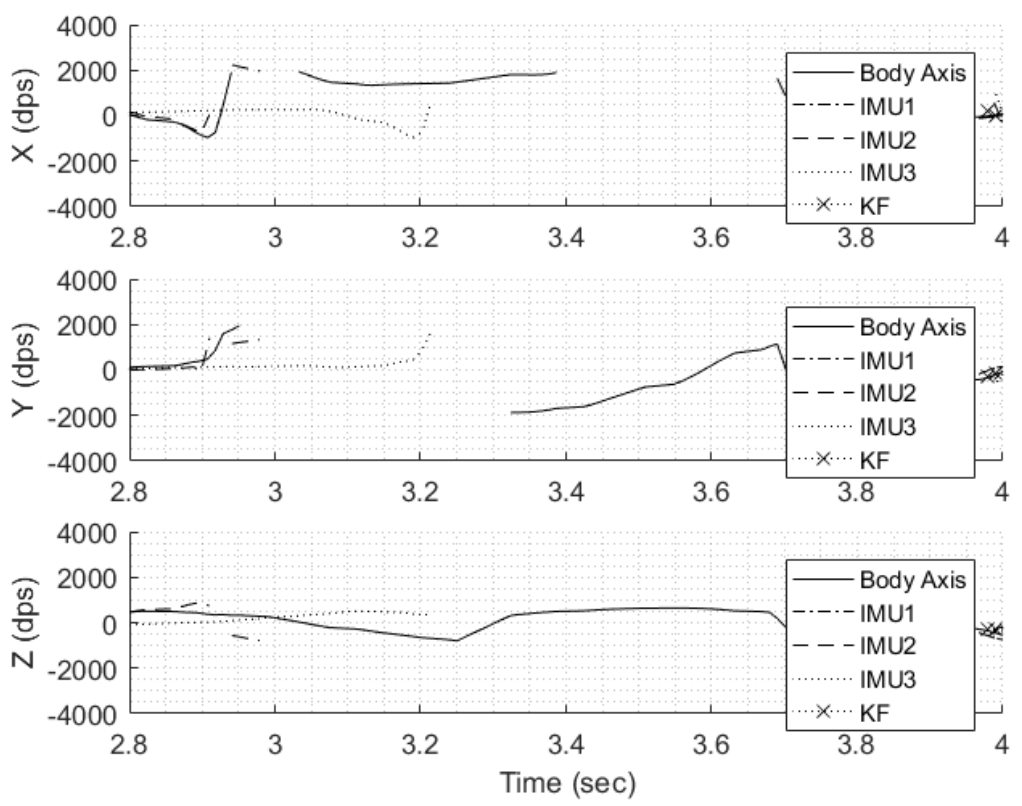

Figure 3.37: Run 5: Converted IMU data and Kalman Filter data fit 


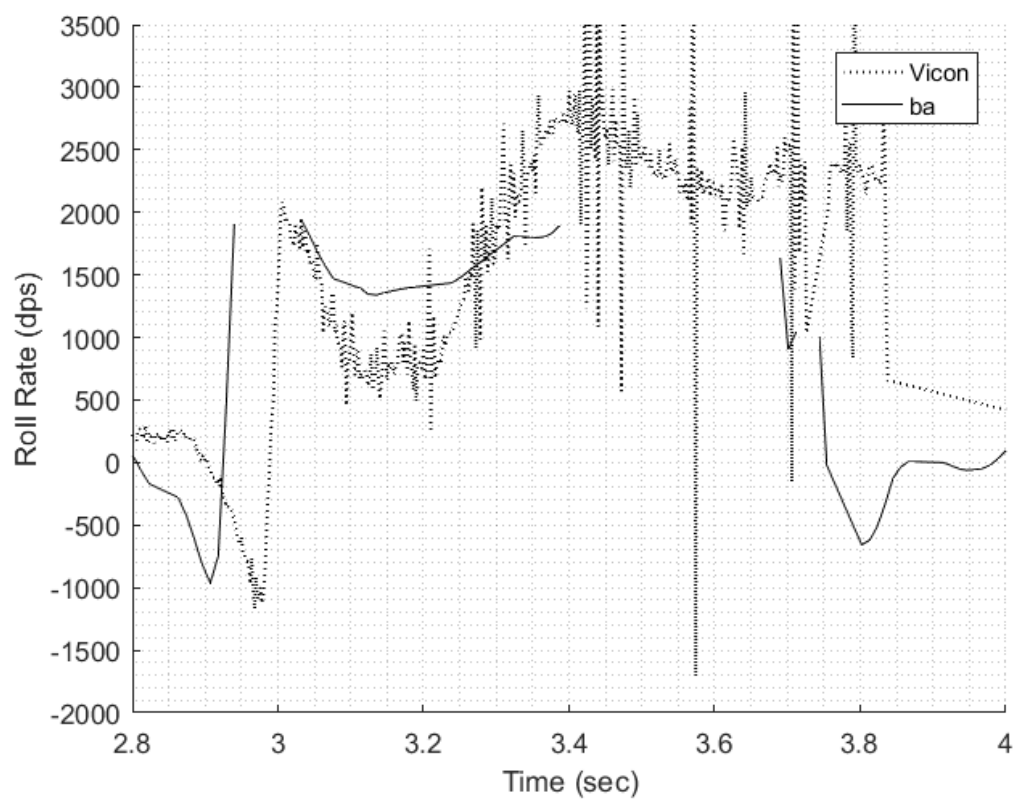

Figure 3.38: Run 5: Rotation about the $\mathrm{x}$ axis 


\subsection{Discussion}

Test runs two and four had skewed IMUs that experienced rotation rates in their local reference frame that stayed at or around the saturation limit. These instances of a skewed IMU that had all three axes unsaturated allowed for those rotation rates to be transformed back to the body axis. Figures 3.11 and 3.26 both show their yaw axes with rotation rates above the two thousand degree per second saturation limit. The rotation rates experienced by the body axis in both test runs were approximately twenty-five hundred degrees per second, which the IMU aligned with the body axes would not have been able to record by itself.

Test runs three and five had skewed IMUs that hard partially saturated values in their respective reference frames during their flights. That meant their values couldn't be transformed back to the body axis using a single rotation matrix. In order to transform the data from the skewed IMUs in these cases, the augmented rotation matrix had to be used. However Figures 3.23 and 3.37 show that there were no saturated rotation rates that were recovered.

Test runs two and four had success in accomplishing the research goal but test runs three and five did not. Test runs three and five required the use of the augmented rotation matrix which took unsaturated rotation rates from more than one skewed IMU and attempted to transform them back to the common body axis reference frame. The failure to utilize the augmented rotation matrix successfully was believed to be in part from the relative shift in time between IMUs. When taking unsaturated values from multiple skewed IMUs, the difference in time was introduced into the mathematical relationship. For example, when using the augmented rotation matrix at time 2.25 seconds shown in figure 3.4, the wrong values were used as the difference in time can be seen.

The four IMU sensors were initially synchronized as substantiated in section 3.4 Flight Test. Figure 3.16 shows the shift in time between signals over the duration of the flight test. Additionally, it can be seen in Figure 3.24 that after initial synchronization that the magnitude of spikes of acceleration occur at different times, indicating that the measurement of the same flight characteristic took place at different times.

It was believed that the accelerations acting on the IMU sensor boards during flight was a potential cause for the difference in timing that occurred. Research looking into this [22] substantiates this as a possible cause. Initial calibration work with the sensor that took place in the lab did not indicate this particular behavior. The sensors were inserted in the flight vehicle payload and placed in a centrifuge to compare known rotation rates to the measured rates of the sensors. There were biases present which matched with known biases from the sensor documentation. However there 
was not a temporal difference in sensor readings over the duration of the lab calibration. That lead to the belief that the behavior noted during actual flight tests were caused by factors not seen in calibration, in this case that could potentially be the accelerations that acted on the flight vehicle. Future work with low cost sensors would benefit from characterizing the effect of accelerations and shocks acting on the sensors by a dynamic calibration setup.

There are possible methods to account for the change in time if the discrepancies were caused by external factors. There are sensors advertised as high shock resistant, which could provide resistance to accelerations altering the crystal oscillators. There are also external hardware timing circuits that could be applied. In the specific case of the research performed, their may be limited space to employ additional hardware, however timing IC (integrated circuit) chips would be a possible solution. They could provide an external and stable time stamp signal used to align data coming in from multiple IMU sensors. Irregardless if the external timing chips cannot provide the exact sample rate required, they would still provide a consistent signal which could be used to piece the separate IMU data back together.

The individual sensors have relatively good performance shown in section 3.3 Uncertainty Analysis with $\pm 4 \%$ relative uncertainty.However the largest additional source of uncertainty was from the time dilation between IMU sensors. It was difficult to quantify in a rigorous manner how the uncertainty propagated through the mathematical reference frame conversion. Because the rotation rates were high at certain times, small differences in time made a significant difference in rotational rates used to convert back to the common body axis reference frame.

Another point worth noting is the importance the skew angles played. The capability of the system to take advantage of the relative difference in coordinate frames depended heavily on the angles which described the skewed IMUs. The construction of the cost function used in the genetic algorithm shaped the skew angles. The data created for the genetic algorithm allowed the skew angles be tailored to get the most performance out of the skewed configuration. The Monte Carlo simulation was run using the designed skew angles and predicted a high probability of recovery. Considering all possible permutations of saturation showed a possible recovery rate approximately $94 \%$ of the data in Figure 2.4. Further inquiry was done into the statistics and was shown in Figure 2.5. Of the total number of data points, $37 \%$ of those had one saturated value on the body reference frame. The recovery rate predicted for only cases having one saturated value was $98 \%$. Of the total number of data points, $37 \%$ had two saturated values on the body reference frame. Of those, $92 \%$ were predicted to be recovered. Finally, of the total number of data points, $12 \%$ had all three values saturated on the body reference frame. $85 \%$ of those cases were technically recoverable. Although 
there was significant confidence predicted in recovering saturated rotation rates, the experiment did not perform to the same level predicted.

Test run 5 is a good comparison to the predicated data because, as can be seen in Figures 3.27 and 3.35 , they have approximately $50 \%$ of their data above the saturation limit of the sensors. The data created for the Monte Carlo simulation also had $50 \%$ of the data beyond the saturation limit. Despite the near similar behavior in experimental data and simulation data, the actual recovered rotation rates were significantly lower. Considering all the data from the five test runs, approximately $35 \%$ of the experimental data was above the saturation limit whereas the total simulated data had $50 \%$ above the saturation limit. Considering that more that half the experimental data was below the saturation limit, it would have been expected to have a good performance in recovering the saturated values, however, that was not the case. The discrepancy between simulation and experiment was believed to be based on the reasons explained above with the time difference when using the augmented rotation matrix and not necessarily the data the genetic algorithm was based on. This belief was based on the mathematically rigorous formulation of reference frame transformation. And was further shown in the additional inquiry into the Monte Carlo simulation data that showed statistically the transformation mathematics does allow recovery of saturated values.

To the best of the author's knowledge, previous work done with a multi sensor skewed configuration revolved around increasing the performance of the sensor measurement in regards to uncertainty. However, no work was found in the literature with a skewed system to leverage the relative difference in coordinate frames to extend the dynamic range of the sensor it self. This is particularly useful when utilizing low-cost sensors that may not have the capabilities required, and it is clear from the results of the described efforts that the SRIMU can in fact increase the effective dynamic range of single sensor. 


\section{Chapter 4}

\section{Conclusions \& Future Development}

The results of the experiment were compared against the goals of providing critical backup data and extending the dynamic range of a low cost MEMS gyroscope. There was success in extending the dynamic range of the sensors in specific cases discussed in the prior section. However there was not success in extending the dynamic range of the sensor when using the augmented rotation matrix. Due to the partial success of recovering saturated rotation rates, there was not a significant confidence for the system to provide critical backup data to the flight vehicle.

It was believed the inability of the augmented matrix to recover saturated rotation rates was due to the discrepancy in time between IMUs which increased after initial synchronization. Therefore it is recommended that future attempts to extend the dynamic range of a MEMS gyroscope in a skewed redundant configuration focus on ensuring the synchronization between IMUs is accurate. Additionally, it was believed the accelerations experienced during the test flights acted upon the crystal oscillators, changing the timing of the sensors. In order to use the effort described in this work more effectively, dynamic calibration exposing the sensors to high accelerations and shocks should be performed to characterize and account for the effects experienced in this work. Using an external timing source to synchronize the separate sensors would also help mitigate these effects. 


\section{Bibliography}

[1] U.S. Dynamics, 425 Bayview Ave. Amityville, NY 11701, Spinning Mass Mechanical Gyroscopes, August 2006.

[2] Veljović, L., "History and present of gyroscope models and vector rotators," vectors, Vol. 2, 2010, pp. 1.

[3] Osman, A., Wright, B., Noureldin, A., and El-Sheimy, N., "Multi-sensor inertial navigation systems employing skewed redundant inertial sensors," ION GNSS 19th International Technical Meeting of the Satellite Division, 2006, pp. 26-29.

[4] Sukkarieh, S., Gibbens, P., Grocholsky, B., Willis, K., and Durrant-Whyte, H. F., "A low-cost, redundant inertial measurement unit for unmanned air vehicles," The International Journal of Robotics Research, Vol. 19, No. 11, 2000, pp. 1089-1103.

[5] Guerrier, S., "Improving accuracy with multiple sensors: Study of redundant MEMS-IMU/GPS configurations," Proceedings of the 22nd international technical meeting of the Satellite Division of the Institute of Navigation (ION GNSS 2009), No. May, 2009, 2009, pp. 3114-3121.

[6] Pejsa, A. J., "Optimum skewed redundant inertial navigators," AIAA Journal, Vol. 12, No. 7, 1974, pp. 899-902.

[7] Colomina, I., Giménez, M., Rosales, J., Wis, M., Gómez, A., and Miguelsanz, P., "Redundant IMUs for precise trajectory determination," XXth ISPRS Congress, Citeseer, 2004, p. 7.

[8] Jafari, M., "Optimal redundant sensor configuration for accuracy increasing in space inertial navigation system," Aerospace Science and Technology, Vol. 47, 2015, pp. 467-472.

[9] Lee, W. and Park, C. G., "A fault detection method of redundant imu using modified principal component analysis," International Journal of Aeronautical and Space Sciences, Vol. 13, No. 3, 2012, pp. 398-404. 
[10] Boström, H., Andler, S. F., Brohede, M., Johansson, R., Karlsson, A., Van Laere, J., Niklasson, L., Nilsson, M., Persson, A., and Ziemke, T., "On the definition of information fusion as a field of research," 2007.

[11] Galar, D. and Kumar, U., EMaintenance: Essential electronic tools for efficiency, Academic Press, 2017.

[12] Hall, D. L. and Llinas, J., "An introduction to multisensor data fusion," Proceedings of the IEEE, Vol. 85, No. 1, 1997, pp. 6-23.

[13] Zhou, G. and Reichle, S., "UAV-based multi-sensor data fusion processing," International Journal of Image and Data Fusion, Vol. 1, No. 3, 2010, pp. 283-291.

[14] Groves, P. D., Principles of GNSS, Inertial, and Multisensor Integrated Navigation Systems, Artech House, Boston, Massachusetts, 2nd ed., 2013.

[15] White, E. and Rios, J. A., "FAA certification of a MEMS attitude and heading reference system," Proceedings of the 2002 National Technical Meeting of The Institute of Navigation, 2002, pp. 158-169.

[16] Gonzalez, R. and Dabove, P., "Performance Assessment of an Ultra Low-Cost Inertial Measurement Unit for Ground Vehicle Navigation," Sensors, Vol. 19, No. 18, 2019, pp. 3865.

[17] Gross, J. N., Gu, Y., Rhudy, M. B., Gururajan, S., and Napolitano, M. R., "Flight-test evaluation of sensor fusion algorithms for attitude estimation," IEEE Transactions on Aerospace and Electronic Systems, Vol. 48, No. 3, 2012, pp. 2128-2139.

[18] Kim, J.-H., Sukkarieh, S., and Wishart, S., "Real-time Navigation, Guidance, and Control of a UAV using Low-cost Sensors," Field and Service Robotics, Springer, 2003, pp. 299-309.

[19] Wilkins, D., Sir William Rowan Hamilton, Encyclopædia Britannica, inc., 2020.

[20] Hamilton, W. R. and Joly, C. J., Elements of quaternions, Vol. 1, Longmans, Green, and Company, 1899.

[21] Taylor, J. R., "An introduction to error analysis: the study of uncertainties in physical measurements," Univ. Science Books, Sausalito, California, 1997.

[22] Vig, J. R., "The effects of accelerations on precision frequency sources," Tech. rep., US Army Electronics Technology and Devices Laboratory, 1992. 


\section{Appendix A}

Hand Calculation for 2D platform. Markers were considered with their lines of symmetry and potential areas to help add in an asymmetric marker location.

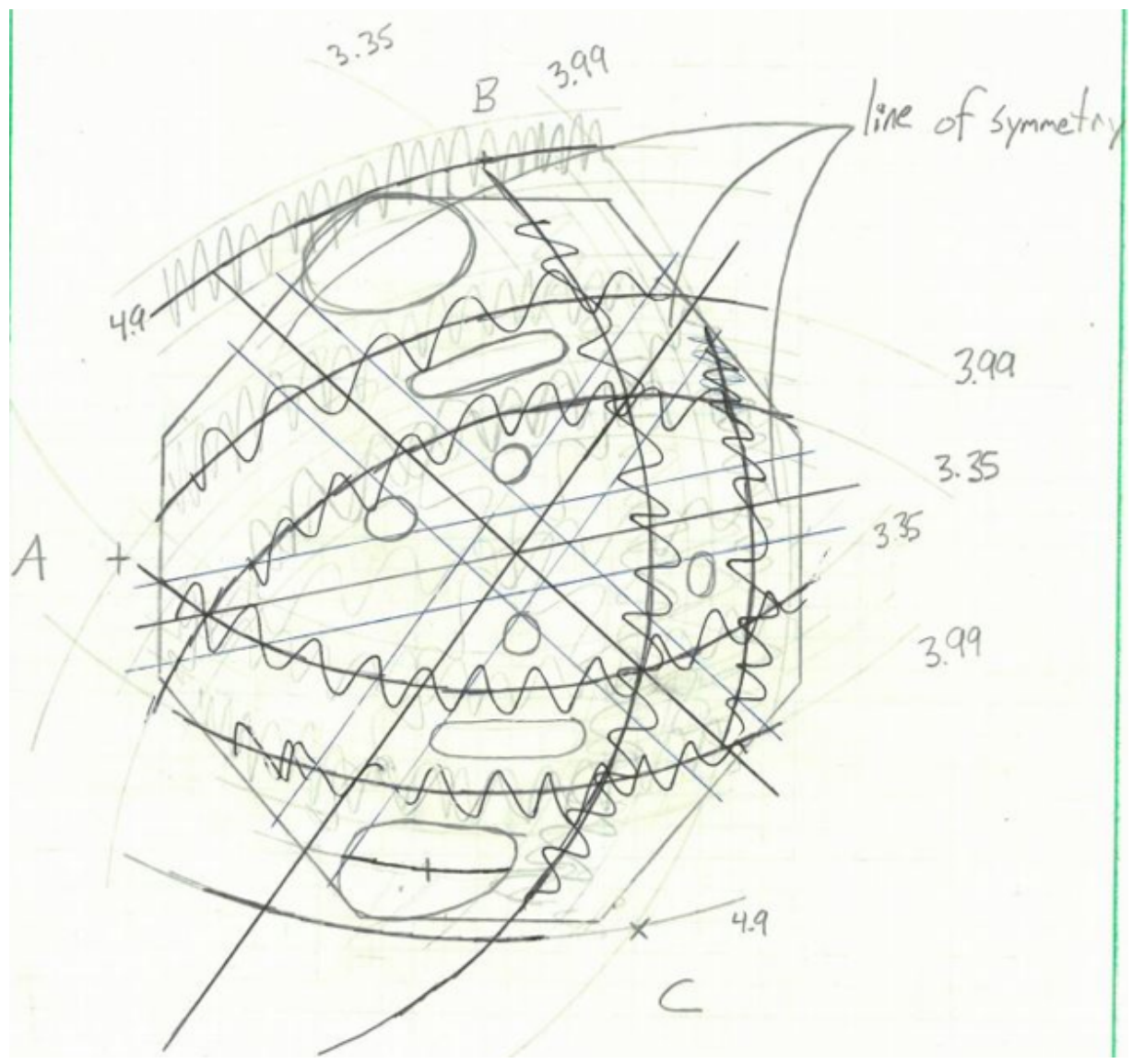

Figure 4.1: Hand drawn calculations for marker placement 


\section{Appendix B}

Table noting distance between Vicon reflective markers to ensure no two distances were the same.

\begin{tabular}{|r|r|r|r|r|r|r|}
\hline & \multicolumn{1}{|c|}{$\mathrm{AB}$} & \multicolumn{1}{c|}{$\mathrm{BC}$} & \multicolumn{1}{c|}{$\mathrm{CA}$} & $\mathrm{AD}$ & $\mathrm{BD}$ & \multicolumn{1}{c|}{$\mathrm{CD}$} \\
\hline $\mathrm{AB}$ & 0 & 1.544 & 0.641 & 0.565 & 1.1215 & 1.914 \\
\hline $\mathrm{BC}$ & 1.544 & 0 & 0.903 & 2.109 & 0.4225 & 3.458 \\
\hline $\mathrm{CA}$ & 0.641 & 0.903 & 0 & 1.206 & 0.4805 & 2.555 \\
\hline $\mathrm{AD}$ & 0.565 & 2.109 & 1.206 & 0 & 1.6865 & 1.349 \\
\hline $\mathrm{BD}$ & 1.1215 & 0.4225 & 0.4805 & 1.6865 & 0 & 3.0355 \\
\hline $\mathrm{CD}$ & 1.914 & 3.458 & 2.555 & 1.349 & 3.0355 & 0 \\
\hline
\end{tabular}

Figure 4.2: Euclidean distance calculation considering all permutations between reflective markers. Measurements were in inches. 


\section{Appendix C}

IMU System of equations that are used to create an augmented rotation matrix.

$$
\begin{gathered}
a_{11} X_{b a}+a_{12} Y_{b a}+a_{13} Z_{b a}=X 1_{\text {local }} \\
a_{21} X_{b a}+a_{22} Y_{b a}+a_{23} Z_{b a}=Y 1_{\text {local }} \\
a_{31} X_{b a}+a_{32} Y_{b a}+a_{33} Z_{b a}=Z 1_{\text {local }} \\
b_{11} X_{b a}+b_{12} Y_{b a}+b_{13} Z_{b a}=X 2_{\text {local }} \\
c_{31} X_{b a}+c_{32} Y_{b a}+c_{33} Z_{b a}=Z 3_{\text {local }} \\
c_{21} X_{b a}+c_{22} Y_{b a}+c_{23} Z_{b a}=Y 3_{\text {local }} \\
b_{21} X_{b a}+b_{22} Y_{b a}+b_{23} Z_{b a}=Y 2_{\text {local }} \\
b_{31} X_{b a}+b_{32} Y_{b a}+b_{33} Z_{b a}=Z 2_{b a}+c_{13} Z_{b a}=X 3_{\text {local }}
\end{gathered}
$$




\section{Appendix D}

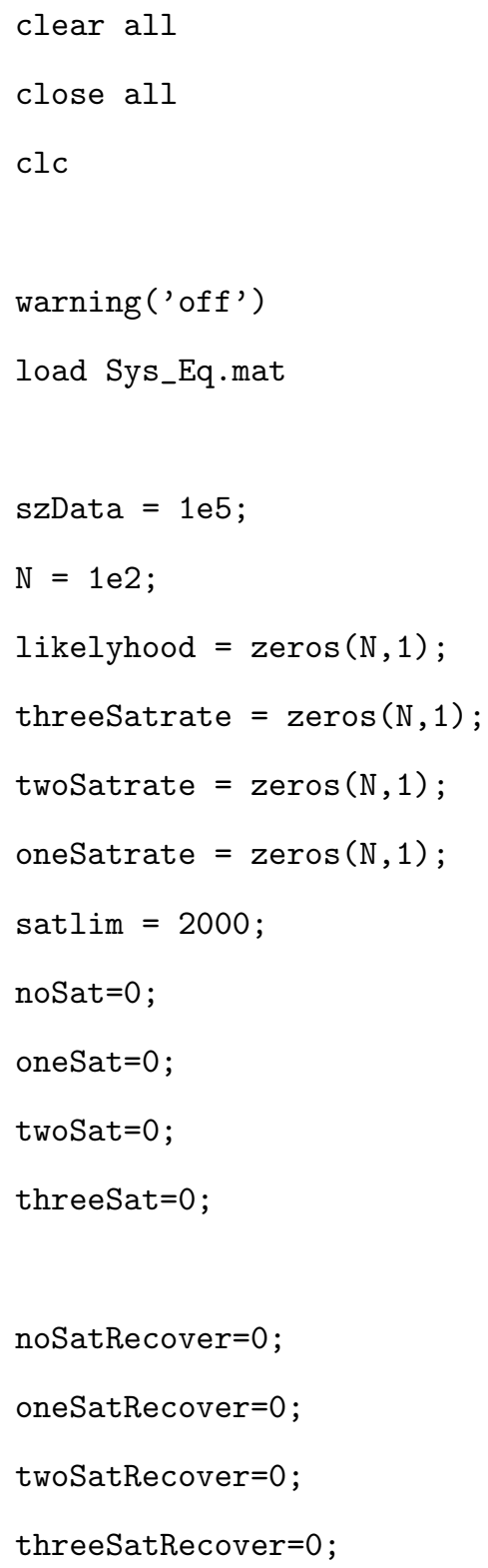




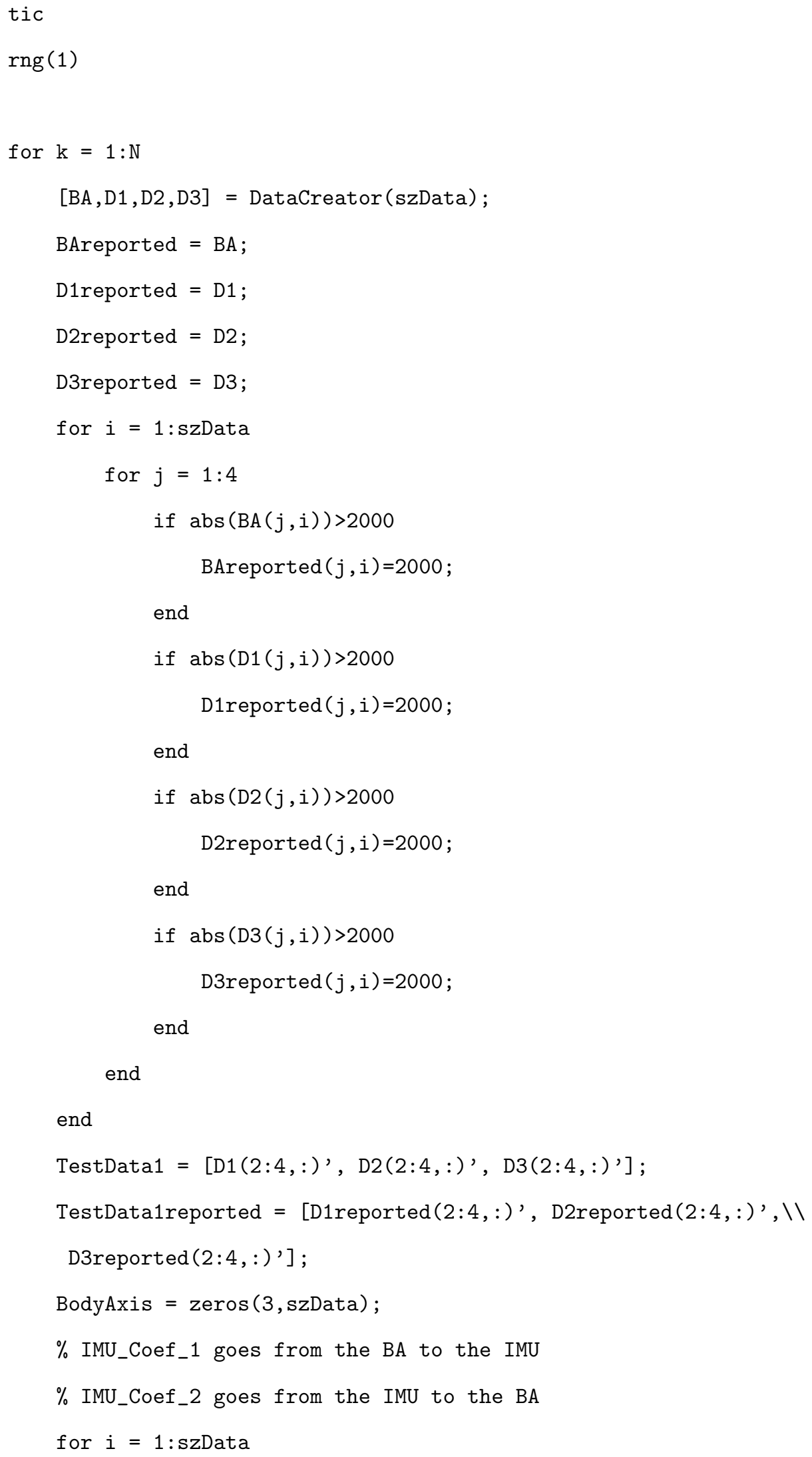




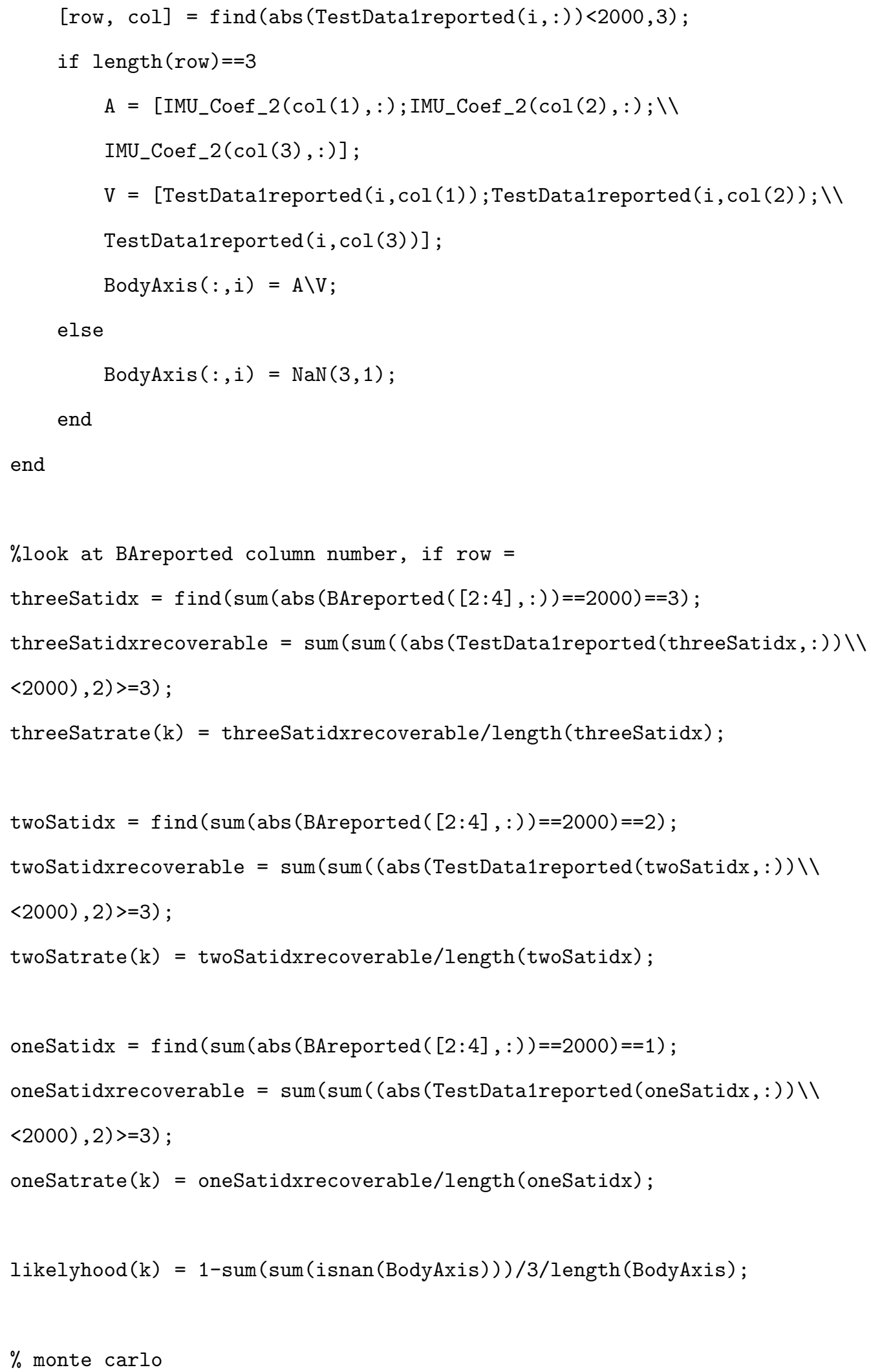




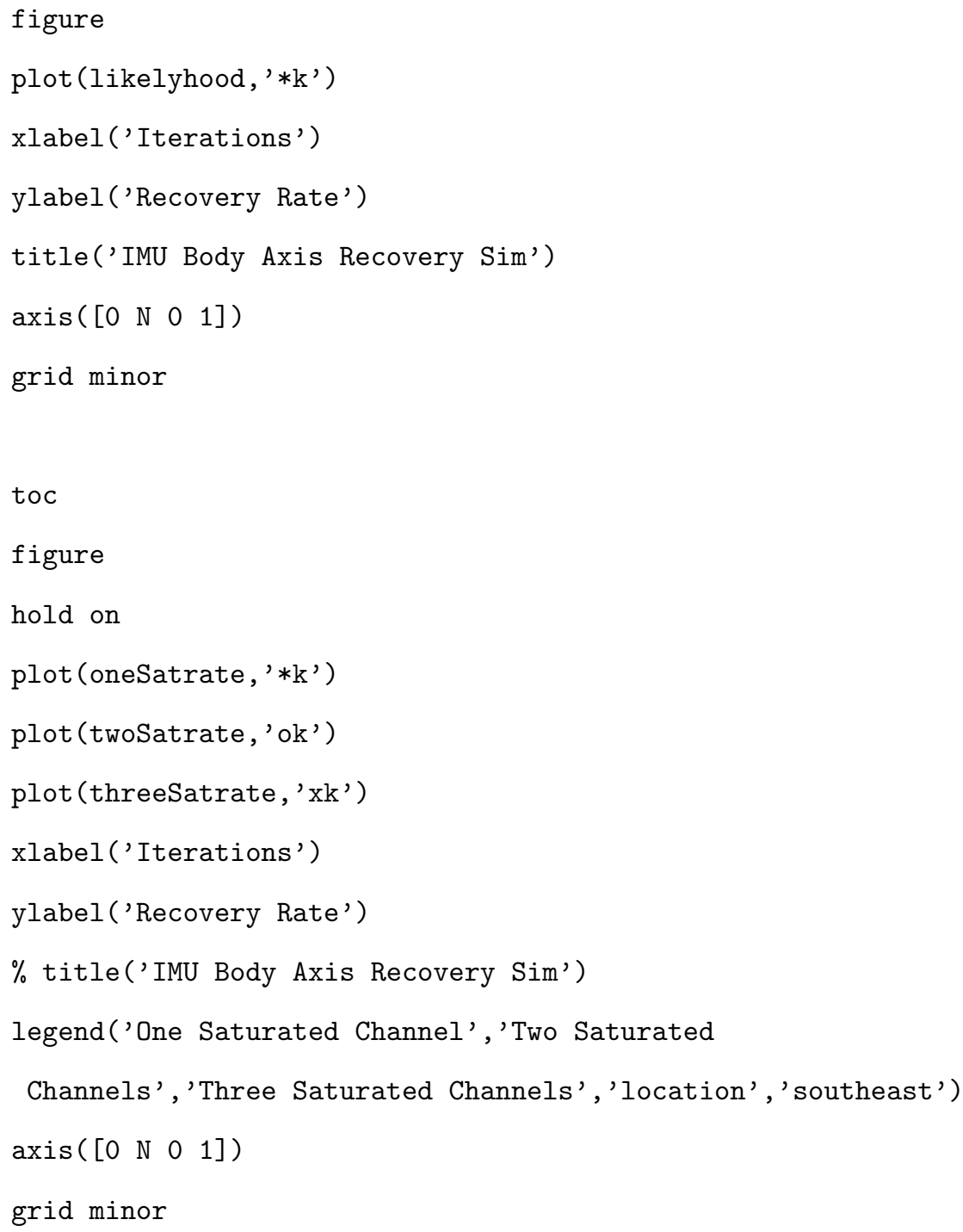

